Aus der Poliklinik für Präventive Zahnmedizin, Parodontologie und Kariologie (Prof. Dr. A. Wiegand)

im Zentrum Zahn-, Mund- und Kieferheilkunde der Medizinischen Fakultät der Universität Göttingen

\title{
Klinische Untersuchung zur Mundgesundheitsbezogenen Lebensqualität (MLQ) und weiterer Einflussfaktoren bei Patienten mit und ohne parodontale Behandlungsbedürftigkeit - Ergebnisse einer praxisbasierten Pilotstudie
}

\section{INAUGURAL-DISSERTATION}

zur Erlangung des Doktorgrades

für Zahnheilkunde

der Medizinischen Fakultät der

Georg-August-Universität zu Göttingen

vorgelegt von

Magdalene Agnes Schoenitz, geb. Kolder

aus

Teschen/ Polen

Göttingen 2018 
Dekan:

Referent:

Ko-Referent/in:

Datum der mündlichen Prüfung: $\quad$ 14.01.2019
Prof. Dr. rer. nat. H. K. Kroemer

Prof. Dr. med. dent. D. Ziebolz, M.Sc.

PD Dr. med. dent. M. Rödiger 
Hiermit erkläre ich, die Dissertation mit dem Titel „Klinische Untersuchung zur Mundgesundheitsbezogenen Lebensqualität (MLQ) und weiterer Einflussfaktoren bei Patienten mit und ohne parodontale Behandlungsbedürftigkeit - Ergebnisse einer praxisbasierten Pilotstudie" eigenständig angefertigt und keine anderen als die von mir angegebenen Quellen und Hilfsmittel verwendet zu haben.

Göttingen, den................... 


\section{Inhaltsverzeichnis}

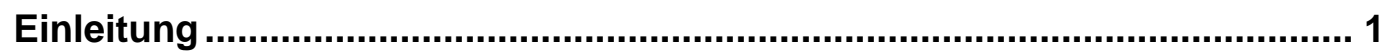

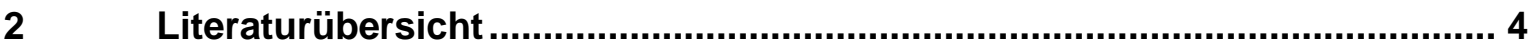

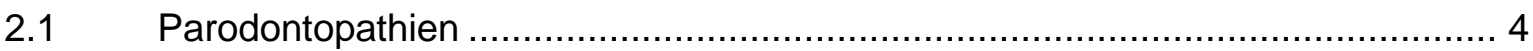

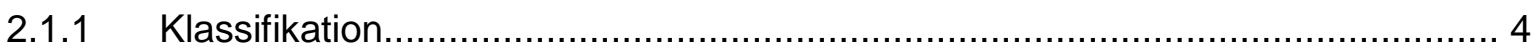

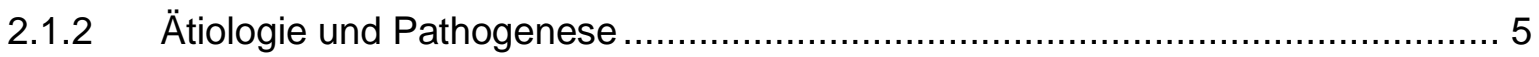

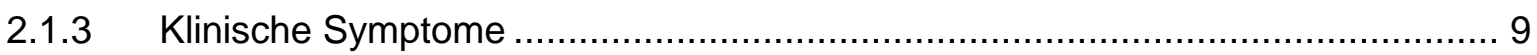

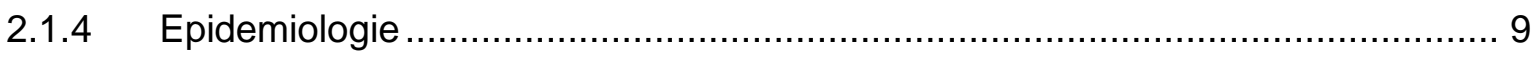

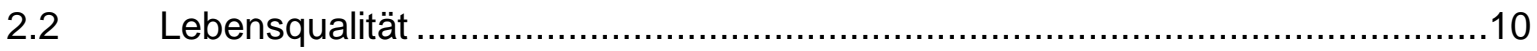

2.2.1 Definition ......................................................................................... 10

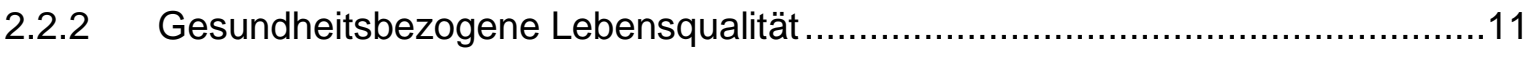

2.2.3 Mundgesundheitsbezogene Lebensqualität (MLQ) ....................................12

2.3 Forschungsergebnisse zum Zusammenhang von Parodontitis und Lebensqualität

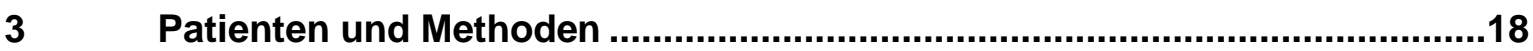

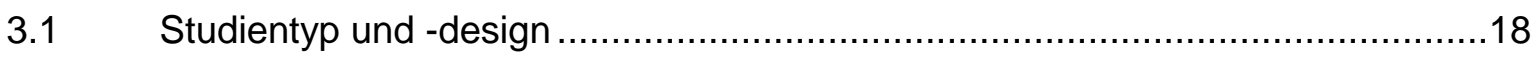

3.2 Gewinnung der Patientenstichprobe ..........................................................19

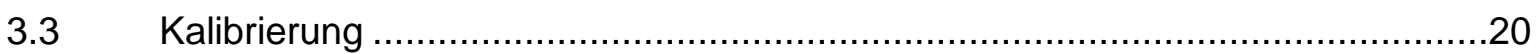

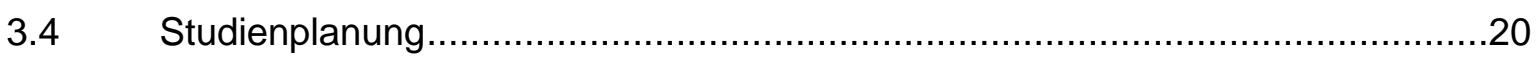

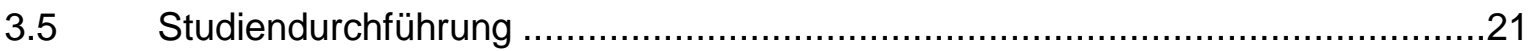

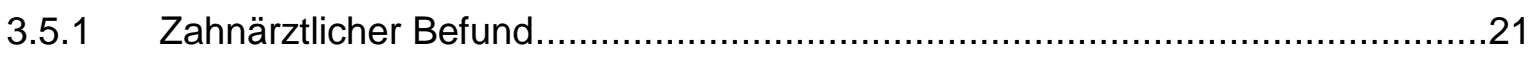

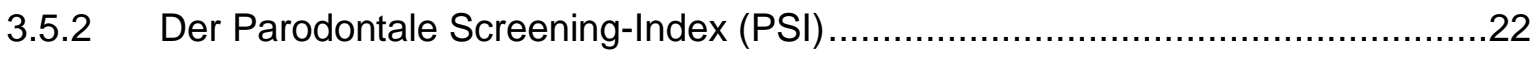

3.6 Fragebögen zur Erhebung des Gesundheitszustands und der Lebensqualität der

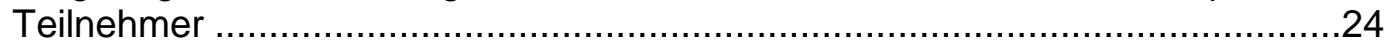

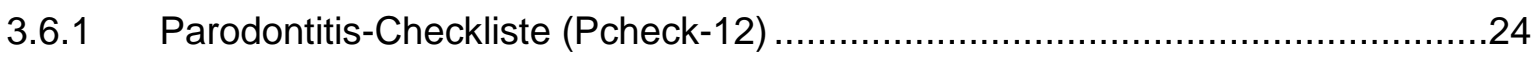

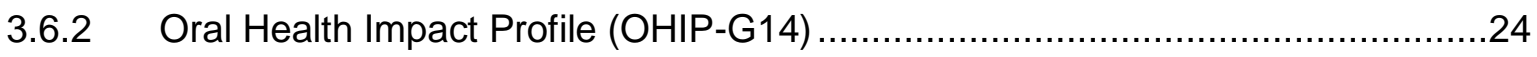

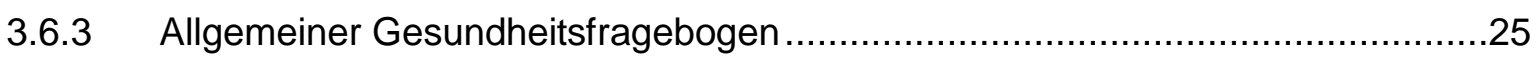

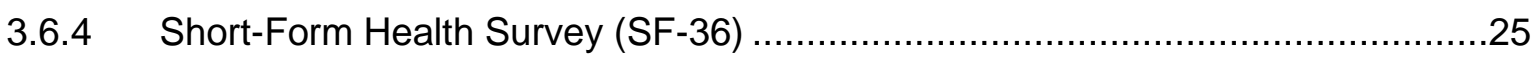

3.6.5 World Health Organization Quality of Life Scale (WHOQOL-BREF) ..................26

3.6.6 Hospital Anxiety and Depression Scale - Deutsche Version (HADS-D) .............27

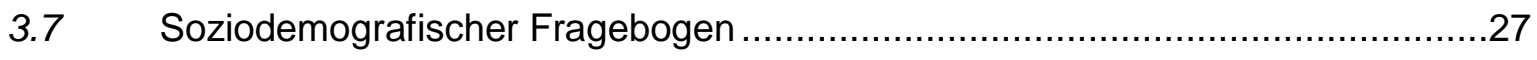

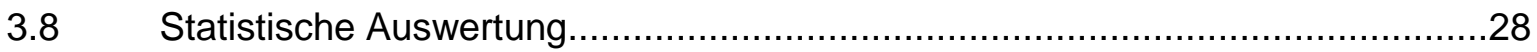

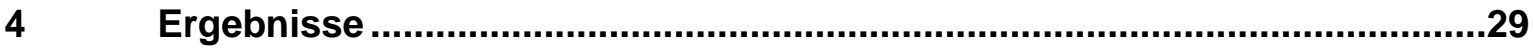

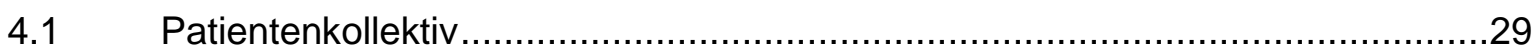

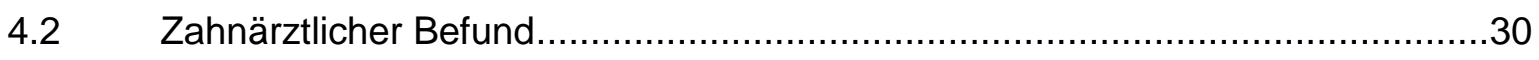

4.2.1 Decayed-Missing-Filled Teeth Index (DMF-T) ........................................30

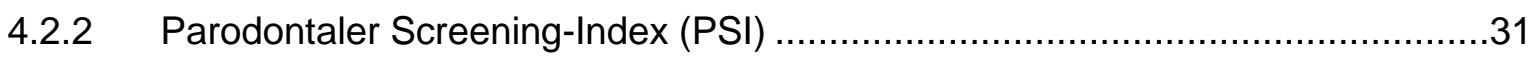

4.3 Befragung zu Gesundheitszustand und Lebensqualität ................................33

4.3.1 Parodontitis-Checkliste (Pcheck-12) ......................................................33

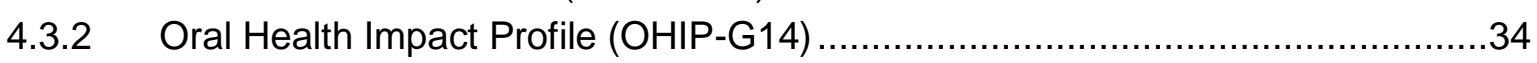




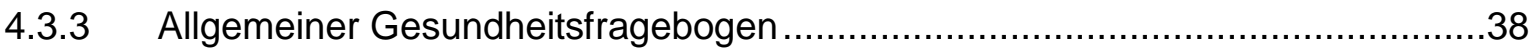

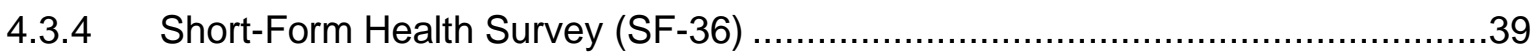

4.3.5 World Health Organization Quality of Life Scale (WHOQOL-BREF) ..................40

4.3.6 Hospital Anxiety and Depression Scale - Deutsche Version (HADS-D) .............41

4.4 Bedeutende Einflussvariablen auf die Lebensqualität .....................................41

4.5 Zusammenfassung der wichtigsten Ergebnisse ............................................48

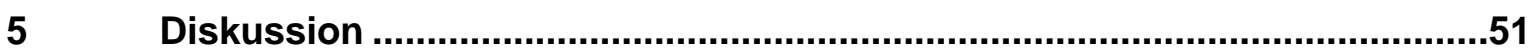

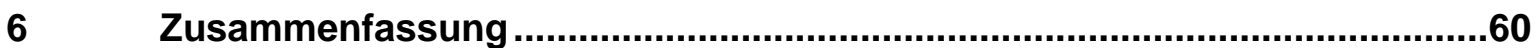

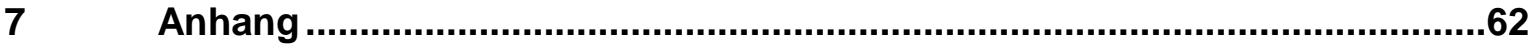

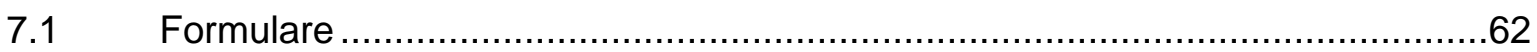

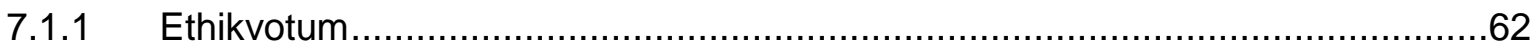

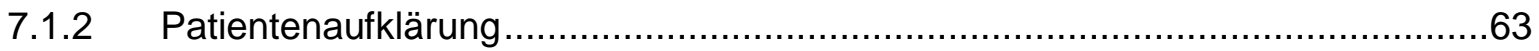

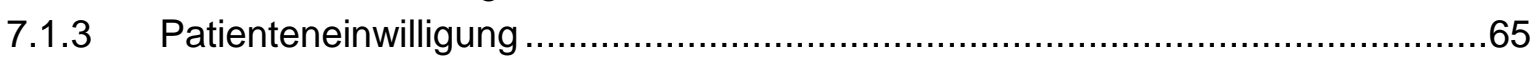

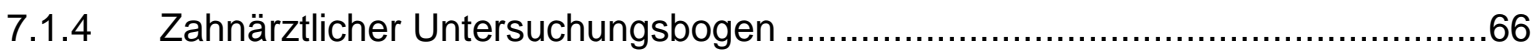

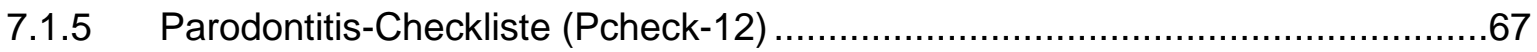

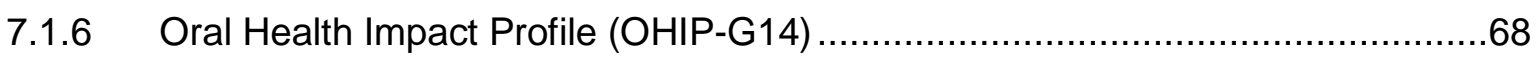

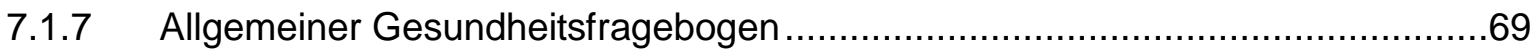

7.1.8 Short-Form Health Survey (SF-36) ........................................................... 71

7.1.9 World Health Organization Quality of Life Scale (WHOQOL-BREF) ....................74

7.1.10 Hospital Anxiety and Depression Scale - Deutsche Version (HADS-D) .............78

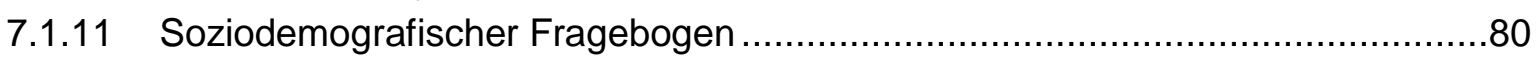

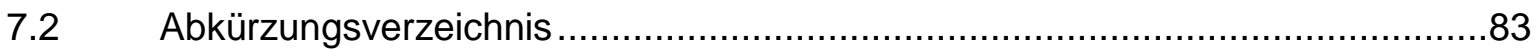

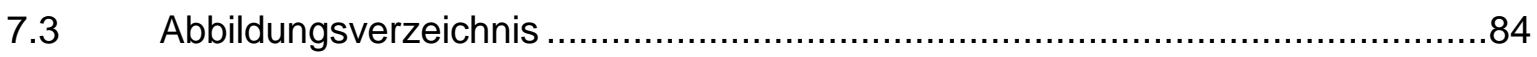

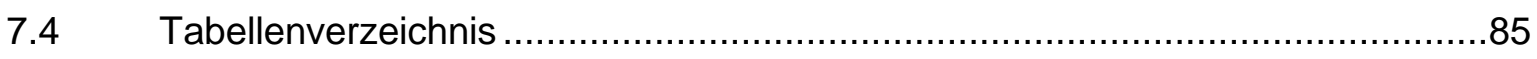

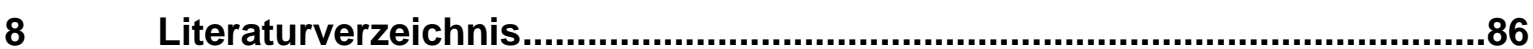




\section{$1 \quad$ Einleitung}

Bei Parodontopathien handelt es sich vorwiegend um bakteriell bedingte Entzündungen des Parodontiums (Listgarten 1986; Listgarten 1987; Löe und Theilade 1965; Wolf et al. 2012). Insgesamt ist die Ätiopathogenese parodontaler Erkrankungen komplex und multifaktoriell. Dabei sind die Prädisposition, der Krankheitsverlauf, der Schweregrad und die Reaktion auf eine Therapie von verschiedenen angeborenen und erworbenen Risikound Umweltfaktoren abhängig (Page 1999; Page und Kornman 1997; Löe et al. 1986).

Nach Haffajee und Socransky (2005) werden für die Entstehung und Progression parodontaler Erkrankungen folgende Faktoren benötigt: 1) die Virulenz parodontaler pathogener Bakterien, 2) lokale Umweltfaktoren, 3) die Empfänglichkeit des Wirtes.

Bislang gibt es nur wenige klinische Studien, die sich mit dem Einfluss der parodontalen Verhältnisse auf die allgemeine und/oder mundgesundheitsbezogene Lebensqualität befassen. Vor allem fehlt es an einer gesamtheitlichen Betrachtung. Forschungsergebnisse, die Aufschluss über die selbstbeurteilte mundgesundheitsbezogene Lebensqualität geben, haben jedoch eine hohe Praxisrelevanz. So können diese z. B. im klinischen Alltag in der Entscheidungsfindung bei unterschiedlichen Therapievarianten, in der Qualitätssicherung und der Public-HealthForschung mit dem Ziel der Weiterentwicklung im Gesundheitswesen eingesetzt werden (Sischo und Broder 2011; John 2004).

Obwohl die Zahl der Parodontitispatienten in Deutschland abnimmt, ist die Parodontitis mit einer Prävalenz von über $50 \%$ in der erwachsenen Bevölkerung immer noch als „Volkskrankheit“ anzusehen. Zudem ist zu vermuten, dass der tatsächliche Behandlungsbedarf durch den demografischen Wandel zukünftig weiter steigen wird (Jordan 2016).

Orale (mundgesundheitsbezogene) Erkrankungen wie Parodontopathien, aber auch Karies und Neoplasien können mehr oder minder große Auswirkungen auf die Lebensqualität sowohl auf physischer, psychischer und sozialer Ebene haben. Die Zahnmedizin ist bislang vorwiegend auf die Bewertung und Therapie oraler Erkrankungen fokussiert und weniger auf die begleitenden psychischen Empfindungen des Patienten (Allen 2003; Locker 2004; Needleman et al. 2004). Demgegenüber nimmt im medizinischen Kontext die Beachtung der Lebensqualität über die gesamte Lebensspanne immer weiter an Bedeutung zu. Ein wesentlicher Grund dafür ist der demografische Wandel in den Industrieländern, der zu einer erhöhten Anzahl älterer Menschen mit chronischen degenerativen Erkrankungen führt. Nicht nur die klinische Feststellung und Behandlung von „Krankheit“, sondern eine ganzheitliche Betrachtung des Menschen inklusive seiner Lebensqualität und neben der 
Verlängerung des Lebens eine Steigerung der "Qualität“ des Lebens sind fundamentale Ziele medizinischer und gesellschaftlicher Interventionen geworden (Müller und von Steinbüchel 2005; Bullinger et al. 2000). Bereits 1946 hat die Weltgesundheitsorganisation (WHO) mit der Definition von Gesundheit als einem „Zustand vollständigen körperlichen, geistigen und sozialen Wohlbefindens und nicht nur das Freisein von Krankheit und Gebrechen" (Weltgesundheitsorganisation - WHO 1948, S. 1) für das Herauslösen aus einer rein biomedizinischen Sichtweise gesorgt.

Das Konzept „mundgesundheitsbezogene Lebensqualität (MLQ)“ beschreibt das subjektive Erleben und Empfinden der eigenen Mundgesundheit durch den Patienten selbst. Sie ist Teil der gesundheitsbezogenen Lebensqualität und diese wiederum Teil der allgemeinen Lebensqualität (John 2005; John und Micheelis 2003). Einschränkungen in der Mundgesundheit können sich negativ auf das Allgemeinbefinden und somit auf die Lebensqualität auswirken (John 2004). Patienten mit oralen Erkrankungen berichten von einer schlechten Mundgesundheit, und es gibt Assoziationen, dass diese die MLQ herabsenken (López und Baelum 2007; Cunha-Cruz et al. 2007; Needleman et al. 2004; Saito et al. 2010). Inwieweit eine Parodontitis die MLQ beeinflusst, ist bisher nicht eindeutig geklärt. Einige Untersuchungen legen eine Assoziation nahe (Bernabé und Marcenes 2010; $\mathrm{Ng}$ und Leung 2006; Cunha-Cruz et al. 2007; Needleman et al. 2004); in anderen Untersuchungen konnten keine Zusammenhänge gefunden werden (Micheelis und Schiffner 2006; Ohrn und Jönsson 2012).

Das Ziel dieser Arbeit war die Durchführung einer Pilotstudie zum Vergleich der mundgesundheitsbezogenen Lebensqualität unter Berücksichtigung der allgemeinen Lebensqualität und der gesundheitsbezogenen Lebensqualität sowie weiterer Einflussfaktoren bei Patienten mit und ohne parodontale Behandlungsbedürftigkeit. Die Lebensqualität mitbeeinflussende Faktoren, wie bestimmte physische und psychische Erkrankungen (z. B. Depressionen, Diabetes mellitus) und orale Faktoren wie Karies, herausnehmbarer Zahnersatz und viele fehlende Zähne, sollten durch strenge Auswahlkriterien minimiert werden bzw. durch spezielle Fragen erkannt werden. Die vorliegende Pilotstudie dient der Überprüfung der Studienparameter für eine nachfolgende großangelegte Hauptuntersuchung.

Folgende Hypothesen wurden formuliert:

I Patienten ohne eine parodontale Behandlungsbedürftigkeit haben eine geringere Beeinträchtigung der mundgesundheitsbezogenen Lebensqualität als Patienten, die eine parodontale Behandlungsbedürftigkeit aufweisen.

II Patienten ohne eine parodontale Behandlungsbedürftigkeit haben eine geringere Beeinträchtigung der krankheitsübergreifenden Lebensqualität als Patienten, die eine parodontale Behandlungsbedürftigkeit aufweisen. 
III Kovariate wie Alter, Angst, Depression beeinflussen die übergreifende wie mundgesundheitsbezogene subjektive Lebensqualität.

Patienten ohne suffizienten, festsitzenden Zahnersatz sowie mit herausnehmbarem Zahnersatz wurden von der Untersuchung ausgeschlossen. 


\section{Literaturübersicht}

\subsection{Parodontopathien}

Bei Parodontopathien handelt es sich um multifaktorielle Erkrankungen, welche sich vorwiegend als bakteriell bedingte Entzündungen des Parodontiums darstellen (Listgarten 1986; Listgarten 1987; Löe und Theilade 1965; Wolf et al. 2012). Diese können auf die Gingiva beschränkt sein (Gingivitiden) sowie die tieferen Strukturen des Parodontiums betreffen (Parodontitiden). Das Parodontium (Zahnhalteapparat) besteht aus der Gingiva, dem Wurzelzement, dem desmodontalem Faserapparat und dem Alveolarknochen. Die Hauptfunktionen liegen in der Verankerung des Zahnes im Knochenfach und dem Schutz des inneren parodontalen Gewebes vor dem Mundhöhlenmilieu (Hellwig et al. 2003; Wolf et al. 2012). Bei der Gingivitis sind die papillären und marginalen Bereiche der Gingiva betroffen. Bei der Parodontitis greift der Entzündungsprozess zusätzlich auf den Alveolarknochen und das Desmodont über und führt zur fortschreitenden Destruktion des Parodontiums und damit zu Knochenabbau, Attachmentverlust und Taschenbildung. Ohne Therapie kann dieses zum Zahnverlust führen (Listgarten 1986; Wolf et al. 2012).

\subsubsection{Klassifikation}

1999 entstand in einem internationalen Arbeitskreis in Oakbrook (Illinois, USA) eine einheitliche internationale Klassifikation der Parodontalerkrankungen und repräsentiert den Konsens der internationalen Gruppe von Experten (Armitage 1999).

Die Parodontopathien werden hiernach acht Kategorien zugeordnet (Deutsche Gesellschaft für Parodontologie 2002):

I. Gingivale Erkrankungen

II. Chronische Parodontitis

III. Aggressive Parodontitis

IV. Parodontitis als Manifestation einer Systemerkrankung

V. Nekrotisierende Parodontalerkrankungen

VI. Abszesse des Parodonts

VII. Parodontitis im Zusammenhang mit endodontalen Läsionen

VIII. Entwicklungsbedingte oder erworbene Deformationen und Zustände 
Bei der Parodontitis sind aggressive und chronische Formen zu unterscheiden (Flemmig 1999; Page 1986). Die häufigste Form aller parodontalen Erkrankungen ist mit rund $90 \%$ die chronische Parodontitis (Wolf et al. 2012). Diese kann in jedem Alter entstehen, tritt jedoch ab dem 35. Lebensjahr am häufigsten auf. Prävalenz und Schweregrad nehmen in der Regel mit dem Alter zu (Deutsche Gesellschaft für Parodontologie 2002; Micheelis und Schiffner 2006). Häufig sind Konkremente vorhanden, und die Plaquezusammensetzung ist variabel. Im Normalfall ist die chronische Parodontitis durch eine langsame Progression gekennzeichnet, Aktivitätsschübe sind jedoch möglich (Deutsche Gesellschaft für Parodontologie 2002; Wolf et al. 2012). Die parodontale Destruktion kann im Zusammenhang mit lokalen Reizfaktoren (z. B. überstehende Kronen- oder Füllungsränder) stehen (Deutsche Gesellschaft für Parodontologie 2002). Zu unterteilen ist die chronische Parodontitis in die lokalisierte Form, wenn bis zu 30\% der Zahnflächen befallen sind, und in die generalisierte Form bei einem Befall von über $30 \%$ der Zahnflächen. Zusätzlich wird der Schweregrad entsprechend dem Attachmentverlust in eine leichte Form (1-2 mm Attachmentverlust), eine moderate Form (3-4 mm Attachmentverlust) und eine schwere Form (über $5 \mathrm{~mm}$ Attachmentverlust) eingeteilt (Deutsche Gesellschaft für Parodontologie 2002; Wolf et al. 2012).

\subsection{2 Ätiologie und Pathogenese}

Nach Löe und Theilade (1965) führt der Verzicht auf Mundhygienemaßnahmen, und damit die Entfernung von Plaque, zu einer Gingivitis. Bei Plaque, heute als Biofilm bezeichnet, handelt es sich um einen zähen, strukturierten, weiß-gelblichen Zahnbelag mit einer dreidimensionalen Struktur - eine Ablagerung von Bakterien, Zellen aus dem Mund und Nahrungsbestandteilen (Glykoproteinen) auf der Zahnoberfläche. Diese Ablagerung kann nur mechanisch entfernt werden (Haffajee und Socranksky 2006; Hellwig et al. 2003; Wolf et al. 2012). Nach Entfernung des Biofilms heilt eine Gingivitis vollständig ab (Löe und Theilade 1965). Verbleibt der Biofilm, kann eine Gingivitis eine Progressionstendenz zur Entstehung einer Parodontitis haben (Page 1986; Lindhe et al. 1973; Listgarten et al. 1985). Ebenso ist eine Parodontitis ohne vorherige Gingivitis möglich (Page 1986). Dabei ist für die Entstehung einer Parodontitis, anders als bei der Gingivitis, nicht allein die Menge des vorhandenen Biofilms entscheidend (Page und Kornman 1997; Löe et al. 1986; Teles et al. 2013).

Trotz hoher Plaqueakkumulation entwickelt nicht jeder Mensch eine Parodontitis mit Attachmentverlust (Baelum et al. 1986; Löe et al. 1986; Teles et al. 2013). Die Zusammensetzung des Biofilms hat hier eine Schlüsselfunktion (Page und Kornman 1997; Löe et al. 1986; Teles et al. 2013). Für die Entstehung und Aufrechterhaltung des Entzündungsprozesses spielen bestimmte Bakterien eine entscheidende Rolle (Listgarten 
1987). In der Mundhöhle befinden sich viele Hundert verschiedene Bakterienspezies, nur wenige davon verursachen Entzündungen.

Der subgingivale mikrobielle Biofilm in parodontalen Taschen stellt ein hochgradig angepasstes Ökosystem mit einer einzigartigen Struktur dar (Darveau et al. 1997; Haffajee und Socranksky 1994; Haffajee und Socranksky 2006). In diesem Biofilm leben die Bakterien in einem Zellverband und agieren als Gemeinschaft (Teles et al. 2013) wobei verschiedene Bakterienspezies jeweils spezielle Aufgaben übernehmen: Bestimmte Spezies sind Frühbesiedler und dienen anderen Organismen als Rezeptoren für die Kolonisation. Es finden Kommunikation und Interaktion zwischen den einzelnen Bakterien, den unterschiedlichen Bakterienspezies und ihrer Umwelt statt (Haffajee und Socranksky 2006). Die parodontale Biozönose hat komplexe Bedingungen für den mikrobiellen Aufbau und unterscheidet sich von Tasche zu Tasche und von Wirt zu Wirt (Haffajee und Socranksky 2005).

Es gibt verschiedene Kombinationen von unterschiedlichen Bakterienspezies, dabei sind manche Kombinationen besonders pathogen (Darveau et al. 1997). Durch diese Mischinfektionen mit vielen möglichen Bakterienkombinationen ist bis heute die Rolle der einzelnen Bakterien bei der Entstehung und Progression der Parodontitis nicht genau geklärt. Für die Forschung erweist es sich als problematisch, dass nicht alle Bakterien kultivierbar sind. Zudem findet ein horizontaler Gentransfer zwischen den einzelnen Bakterienspezies statt und manche Bakterien weisen eine sehr hohe genetische Vielfalt auf, durch die nicht alle Klontypen gleich virulent sind (Haffajee und Socranksky 1994; Teles et al. 2013). Nach Teles et al. (2013) werden mit einer Parodontitis als Leitkeime Porphyromonas gingivalis und Aggregatibacter actinomycetemcomitans assoziiert, weitere sind Eikenella corrodens, Prevotella intermedia, Prevotella nigrescens und Fusobacterium nucleatum. Die alleinige Präsenz pathogener Keime ist jedoch nicht ausreichend für das Auftreten der Erkrankung (Page und Kornman 1997; Teles et al. 2013). Während der bakterielle Biofilm bzw. die damit verbundene genetisch bestimmte Immunreaktion als der primäre ätiologische Faktor fungiert, wird die Pathogenese und damit die Progression durch diverse Wirtsfaktoren beeinflusst. Beim Gesunden besteht ein dynamisches Gleichgewicht zwischen Bakterien und der Immunabwehr. Unter bestimmten Bedingungen können Bakterien indirekt, durch die Aktivierung von Immunzellen, eine destruktive Entzündung verursachen (Darveau et al. 1997; Page und Kornman 1997).

Das Eindringen von Bakterien in den gingivalen Sulkus aktiviert zunächst das unspezifische angeborene Immunsystem. Dabei werden die Bakterien von neutrophilen Granulozyten (PMN) und Makrophagen phagozitiert und damit ihr weiteres Eindringen gehemmt (Wolf et al. 2012). Im für Parodontitis empfänglichen Wirt, wird die erste Immunantwort jedoch überwunden (Page 1999). Die Makrophagen binden bakterielle Antigene und präsentieren 
sie den Lymphozyten, sie bilden dadurch ein wichtiges Bindeglied zum spezifischen, erworbenen Immunsystem (Wolf et al. 2012; Page 1999).

Durch ein zunehmendes Eindringen von Bakterien in das subepitheliale parodontale Gewebe stimuliert das bakterielle Endotoxin, das Lipopolysaccharid, Makrophagen und Gewebszellen dazu, Zytokine auszuschütten und damit eine lokale Entzündungsreaktion auszulösen. Dabei findet eine vermehrte Sekretion proinflammatorischer und kataboler Zytokine, insbesondere Interleukin-1 und -6 und den Tumornekrosefaktor alpha, statt. Diese aktivieren wiederum die Freisetzung von Prostaglandinen und gewebeabbauenden Matrixmetalloproteinasen (MMP) durch Fibroblasten und Granulozyten und verändern das Verhalten und den Stoffwechsel der Zellen im Entzündungsgebiet (Wolf et al. 2012; Page 1999). Dabei ist, trotz des hohen schädigenden Potenzials der Virulenzfaktoren pathogener Bakterien, fast ausschließlich diese entzündliche Immunantwort des Wirts für den parodontalen Knochen- und Weichgewebsabbau verantwortlich (Wolf et al. 2012; Teles et al. 2013).

Die Abbildung 1 zeigt das Zusammenwirken verschiedener Faktoren auf die Ätiopathogenese der Parodontitis (Page und Kornman 1997).

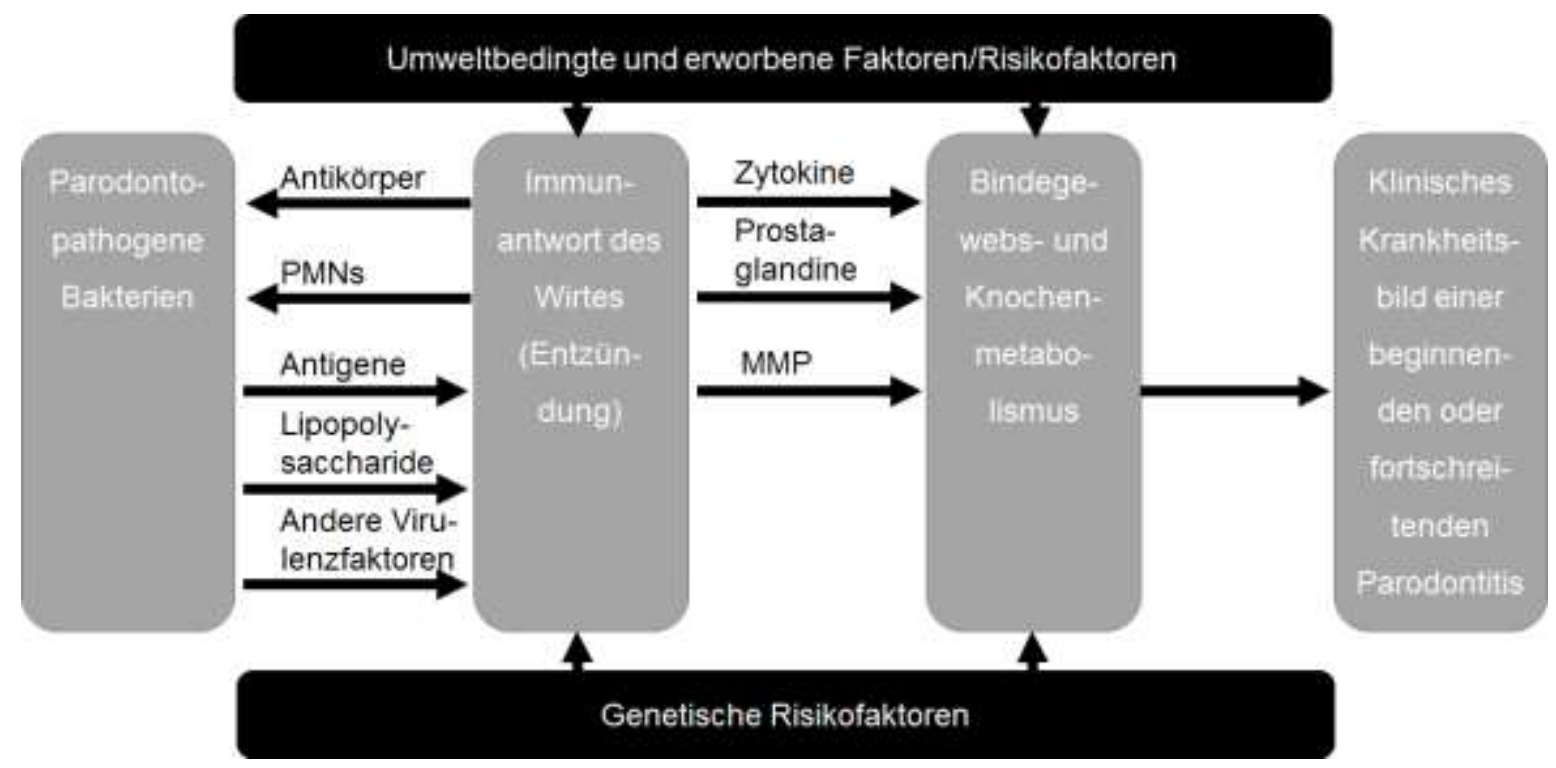

Abbildung 1: Ätiologie und Pathogenese der Parodontitis nach Page und Kornman $(1997$, S. 11)

Den Verlauf und Schweregrad können individuelle Risikofaktoren, wie Systemerkrankungen (z. B. Diabetes mellitus, HIV-Infektion), Lebensgewohnheiten (z. B. Tabakkonsum), sozioökonomische Faktoren und psychische Aspekte (z. B. emotionaler Stress) beeinflussen (Deutsche Gesellschaft für Parodontologie 2002; Wolf et al. 2012). Risikofaktoren schädigen weniger das Parodont direkt (eine Ausnahme stellt hier Rauchen dar), sondern beeinflussen den Immunstatus negativ, so dass das Gleichgewicht zwischen 
Bakterienangriff und Wirtsantwort empfindlich gestört wird (Wolf et al. 2012). Diabetes mellitus und Rauchen bilden die stärksten erworbenen Risikofaktoren (Page 1999). 


\subsubsection{Klinische Symptome}

\subsubsection{Gingivitis}

Klinische Symptome einer Gingivitis entstehen als Konsequenz einer chronischen Entzündung. Das erste signifikante Symptom ist eine schwache Blutung als Reaktion auf einen leichten Reiz, wie zum Beispiel das vorsichtige Sondieren. Beim Fortschreiten der Gingivitis wird die Blutung stärker und eine deutliche Rötung und ödematöse Schwellung können auftreten. Die schwerste Form der Gingivitis kann durch Spontanblutungen, starke Rötungen und hyperplastische, ödematöse Schwellungen bis hin zu Ulzerationen gekennzeichnet sein. Hierbei treten in der Regel keine Schmerzen auf mit Ausnahme einer Sonderform der akuten nekrotisierenden ulzerösen Gingivitiden (ANUG) ( $\mathrm{Ng}$ und Leung 2006; Wolf et al. 2012).

\subsubsection{Parodontitis}

Zusätzlich zu den entzündungsbedingten Symptomen der Gingivitis kommen bei einer Parodontitis weitere klinische und röntgenologische Symptome als Folge der Gewebedestruktion hinzu ( $\mathrm{Ng}$ und Leung 2006; Wolf et al. 2012). Obligates Hauptsymptom ist hierbei der Attachmentverlust. Dieser ist durch die Distanz zwischen der SchmelzZement-Grenze und dem gingivalen Taschenboden definiert (Glavind und Löe 1967; Hellwig et al. 2003; Wolf et al. 2012). Zum Attachmentverlust kommt es durch den Abbau des Alveolarknochens und die Ausbildung einer (echten) parodontalen Tasche aufgrund einer Tiefenproliferation des Saumepithels und dessen Veränderung in ein Taschenepithel. Weitere Begleitsymptome der Parodontitis können sein: Schrumpfung der Gingiva mit Freilegung der Zementoberfläche, Austritt von Exsudat oder Eiter, Taschenabszesse, Fisteln und Zahnwanderungen, -kippungen, -elongationen und -lockerungen bis hin zum Zahnverlust (Wolf et al. 2012).

Subjektive, für den Patienten wahrnehmbare Symptome beschränken sich in der Regel auf die Blutung, Rötung und Schwellung der Gingiva. Veränderungen der Zahnstellung, starke Zahnlockerungen, Zahnverlust und persistierender Mundgeruch treten im weit fortgeschrittenen Stadium auf ( $\mathrm{Ng}$ und Leung 2006).

\subsubsection{Epidemiologie}

Zwischen den beiden deutschen Mundgesundheitsstudien DMS IV (2005) und DMS V (2014) hat sich die Prävalenz von Parodontalerkrankungen verringert.

2005 hatten mehr als 70\% der über 35-Jährigen eine Parodontitis, bis 2014 sank die Prävalenz auf $52 \%$. Die Prävalenz einer schweren Parodontitis hat sich bei Erwachsenen (35-44 Jahre; DMS IV: 17\%; DMS V: 8\%) wie auch bei Senioren (65-74 Jahre; DMS IV: 44\%; DMS V: 20\%) seit 2005 halbiert (Micheelis und Schiffner 2006; Jordan 2016). 
Insgesamt leiden 43\% der Erwachsenen an einer moderaten und $8 \%$ an einer schweren Parodontitis (Jordan 2016). Mit dem Alter nehmen Prävalenz, sowie Umfang und Schweregrad der Parodontitis zu (Flemmig 1999), was sich in den Erkrankungszahlen der Senioren widerspiegelt: $45 \%$ weisen eine moderate und $20 \%$ eine schwere Parodontitis auf (Jordan 2016).

Zudem zeigen sich regionale und geschlechtsspezifische Unterschiede. Die Prävalenz für eine schwere Parodontitis ist bei Erwachsenen in Ost-Deutschland (22\%) verglichen mit West-Deutschland (16\%) höher. Bei Senioren zeigt sich eine ähnliche Verteilung (West: 41\%; Ost: 47\%). Ebenso zeigen Männer gegenüber Frauen eine signifikant höhere Prävalenz für eine Parodontitis. Bei den männlichen Erwachsenen sind es $21 \% \mathrm{im}$ Vergleich zu $14 \%$ bei den weiblichen und bei den männlichen Senioren $48 \%$ im Vergleich zu 36\% bei den weiblichen (Holtfreter et al. 2010).

Im internationalem Vergleich mit den USA hat die deutsche Bevölkerung eine höhere Prävalenz für eine Parodontitis (USA: 62\% zu Deutschland: 70\%). Bei einer schweren Parodontitis ist dieser Unterschied sogar viel deutlicher (USA: 33\% zu Deutschland: 67\%) (Holtfreter et al. 2012). Zu beachten ist, dass Holtfreter et al. (2012) zwei Studien verglichen haben, bei denen nicht die Allgemeinbevölkerung untersucht worden ist, sondern eine spezielle Probandengruppe mit kardiovaskulären Erkrankungen.

\subsection{Lebensqualität}

\subsubsection{Definition}

Seit Jahrzehnten gewinnt das Konzept Lebensqualität (LQ) im Englischen „Quality of Life (QOL)“ immer weiter an Bedeutung (OECD 2015; Noll 2002; Renneberg und Lippke 2006; Ravens-Sieberer und Cieza 2000; Costanza et al. 2008; Ebbinghaus et al. 2006). Der Begriff Lebensqualität ist eng mit den Begriffen Zufriedenheit (englisch: Satisfaction), subjektives Wohlbefinden (englisch: Subjective Well-Being) und Glück (englisch: Happiness) assoziiert (Costanza et al. 2008). Die Lebensqualitätsforschung stellt ein interdisziplinäres Forschungsfeld zahlreicher Wissenschaften dar, neben der Medizin vor allem der Soziologie, Psychologie, Philosophie, Ökonomie, Politik und Religion.

Personen- und umweltbezogene Faktoren haben mittelbaren und unmittelbaren Einfluss auf die (subjektiv empfundene) Lebensqualität (Nussbaum und Sen 1993; Ravens-Sieberer und Cieza 2000; Costanza et al. 2008; Ebbinghaus et al. 2006). Bedeutende Umweltfaktoren sind dabei die natürliche und soziale Umwelt, allgemeine Lebenserwartung, Sicherheit, Rechtsstaatlichkeit, persönliche Freiheitsrechte, Bildungsund Berufschancen sowie Möglichkeiten zur Selbstverwirklichung. $\mathrm{Zu}$ den personenspezifischen Faktoren zählen Zufriedenheit, Gesundheit, Wohlstand/ 
Lebensstandard und sozialer Status. Somit handelt es sich bei Lebensqualität um ein multidimensionales Konstrukt, dessen Teilbereiche über Indikatoren abgebildet werden können (Noll 1999; Costanza et al. 2008). Generell gilt Lebensqualität als ein „Konzept, das sowohl materielle wie auch immaterielle, objektive und subjektive, individuelle und kollektive Wohlfahrtskomponenten gleichzeitig umfasst und das ,besser' gegenüber dem ,mehr' betont“" (Noll 1999, S. 3). 1997 hat die WHO eine Definition der Lebensqualität veröffentlicht: „Lebensqualität ist die subjektive Wahrnehmung einer Person über ihre Stellung im Leben in Relation zur Kultur und den Wertesystemen, in denen sie lebt und in Bezug auf ihre Ziele, Erwartungen, Standards und Anliegen. Es handelt sich um ein breites Konzept, das in komplexer Weise beeinflusst wird durch die körperliche Gesundheit einer Person, den psychischen Zustand, die sozialen Beziehungen, die persönlichen Überzeugungen, und ihre Stellung zu den hervorstechenden Eigenschaften der Umwelt" (Renneberg und Lippke 2006, S. 29).

Eine allgemein anerkannte Definition zum Begriff der Lebensqualität existiert zurzeit jedoch weder auf nationaler noch internationaler Ebene (Konerding 2004; John und Micheelis 2003; Müller und von Steinbüchel 2005; Bullinger et al. 2000).

Zur Erfassung der Lebensqualität haben sich verschiedene Erhebungsinstrumente etabliert. Der Short-Form-36 (SF-36) und der World Health Organization Quality of Life Abbreviated Questionnaire (WHOQOL-BREF) sind Instrumente zur Messung der allgemeinen krankheitsübergreifenden Lebensqualität mit guten psychometrischen Charakteristika (Müller und von Steinbüchel 2005).

\subsubsection{Gesundheitsbezogene Lebensqualität}

Der Begriff Gesundheit weist ebenso wie der Begriff Lebensqualität sowohl physische, als auch psychische und soziale Aspekte auf (Konerding 2004; Bullinger et al. 2000; RavensSieberer und Cieza 2000). Gesundheitsbezogene Lebensqualität ist demnach ein multidimensionales Konstrukt, das sowohl von personenbezogenen Faktoren, wie Persönlichkeit und Verhalten, als auch von umweltbezogenen Faktoren, wie der Befriedigung menschlicher Grundbedürfnisse beeinflusst wird (Allen 2003; John 2005; Prchala 2004; John und Micheelis 2003; John 2004; John et al. 2004). Die gesundheitsbezogene Lebensqualität bildet einen wesentlichen Teil der allgemeinen Lebensqualität (siehe Abbildung 2) (John und Micheelis 2003). 


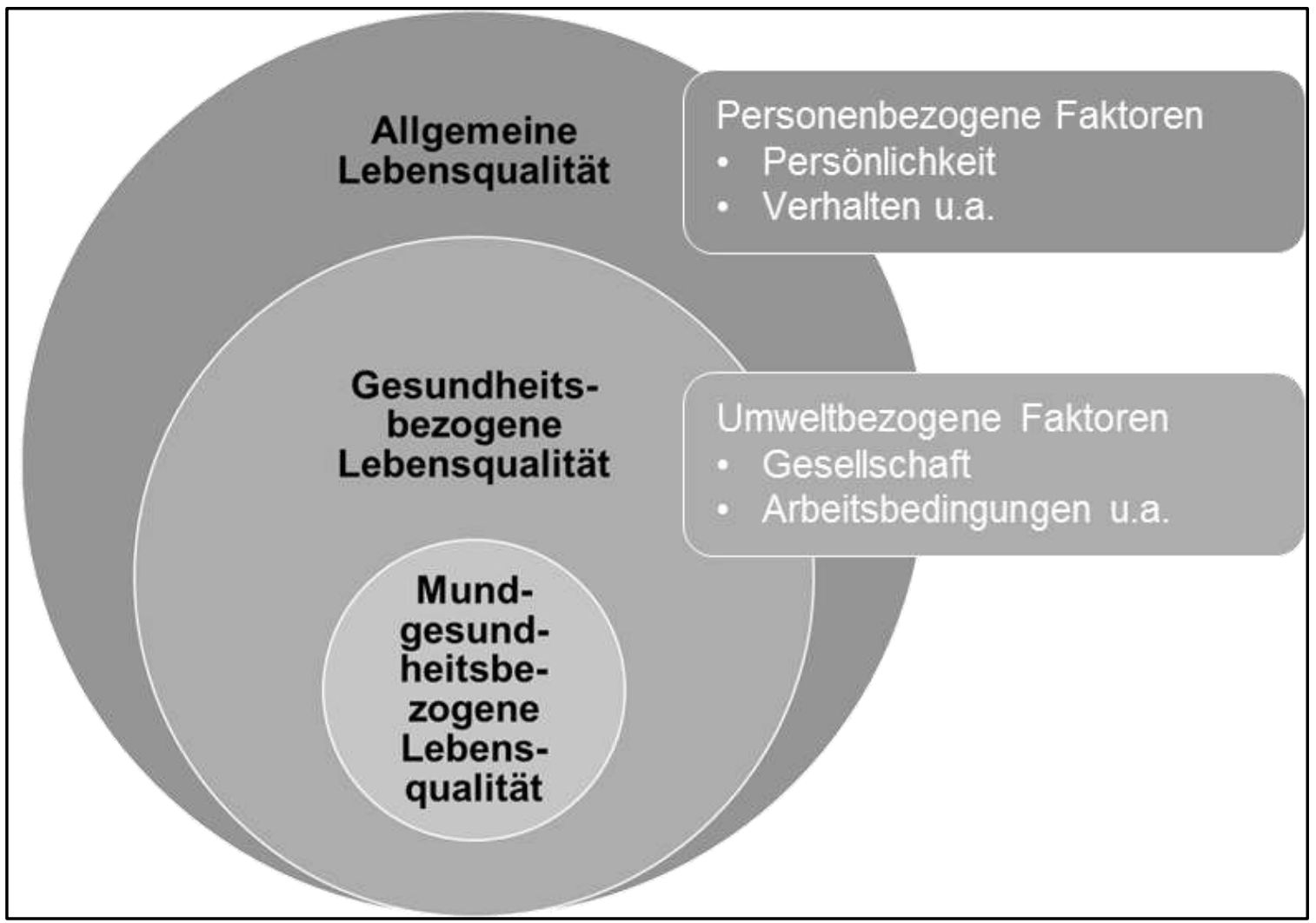

Abbildung 2: Modell zur mundgesundheitsbezogenen Lebensqualität (MLQ), gesundheitsbezogenen Lebensqualität und allgemeinen Lebensqualität nach John und Micheelis (2003)

Seine Dimensionen (körperliche, psychische [emotionale und mentale], soziale und alltagsbezogene Dimension) müssen aus Sicht des Individuums und/oder eines Beobachters in Bezug auf das Wohlbefinden und die Funktionsfähigkeit beurteilt werden (Konerding 2004; Müller und von Steinbüchel 2005; Bullinger et al. 2000).

\subsubsection{Mundgesundheitsbezogene Lebensqualität (MLQ)}

Einschränkungen in der Mundgesundheit können sich negativ auf die (gesundheitsbezogene und allgemeine) Lebensqualität auswirken (Allen 2003; John 2005; Prchala 2004; John und Micheelis 2003; John 2004; John et al. 2004). Die orale Gesundheit kann inklusive der parodontalen Gesundheit als ein elementarer Teil der allgemeinen Gesundheit angesehen werden (Casanova et al. 2014). Die Mundgesundheit bzw. orale Krankheitszustände lassen sich objektiv mit Hilfe einer Vielzahl von Indizes und Messwerten beschreiben. Da jedoch der Befund des Zahnarztes und das Befinden des Patienten nicht immer deckungsgleich sind, ist es hilfreich, einen Einblick in die subjektiv vom Patienten empfundene und beurteilte Mundgesundheit zu erhalten. Die objektive klinische Befundung zusammen mit dem subjektiven Empfinden des Patienten erlauben eine ganzheitliche Betrachtung der gesundheitsbezogenen Lebensqualität des Menschen (Prchala 2004; Sischo und Broder 2011; John et al. 2004). 
Die Mundgesundheitsbezogene Lebensqualität (MLQ) erfasst das subjektive Erleben der eigenen Mundgesundheit durch den Patienten selbst. Nach dem Modell von Inglehart und Bagramian (2002) bilden funktionelle Einschränkungen, chronische und akute Schmerzzustände sowie psychologische und soziale Aspekte die vier Hauptkomponenten der MLQ (s. Abbildung 3). Zur Bestimmung der individuellen MLQ bewertet ein Patient die Beeinflussung des eigenen Wohlbefindens durch diese vier Faktoren.

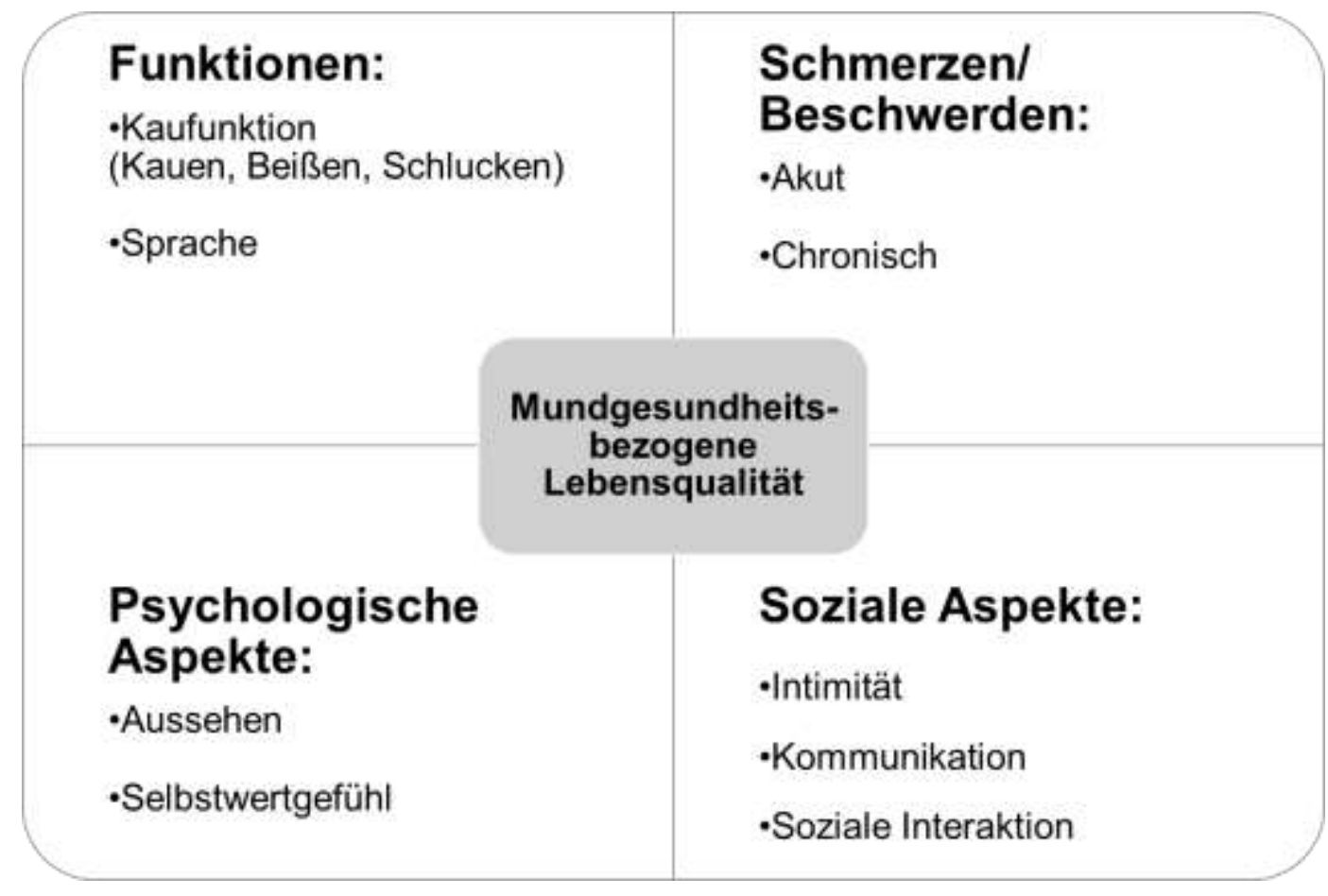

Abbildung 3: Die Hauptkomponenten der mundgesundheitsbezogenen Lebensqualität nach Inglehart und Bagramian 2002, eigene Übersetzung

Nach einer Studie von McGrath und Bedi (2004) in Großbritannien mit 1778 Erwachsenen empfindet eine Mehrheit (75\%) die orale Gesundheit als bedeutend für die allgemeine Lebensqualität. Dabei wird die Lebensqualität am meisten von der „Fähigkeit zu essen“ (23\%) und dem „Komfort“ (17\%) beeinflusst.

Ästhetische Aspekte, die das Aussehen und damit das Selbstwertgefühl beeinflussen, haben durch einen Gesellschaftswandel hin zu postmaterialistischen Werten, wie z. B. Gesundheit, Glück und Kultur, an Bedeutung gewonnen (Inglehart und Bagramian 2002). Die Mundhöhle besitzt somit eine hohe psycho-emotionale Bedeutung und neben den eigentlichen Organfunktionen Kauen und Beißen sind das Wohlbefinden, Gesundheit allgemein und Aussehen als positive Einflussfaktoren der Zähne zu betonen (Micheelis 1997). Mit einem Zahnverlust gehen funktionelle und vor allem im Frontzahnbereich ästhetische und phonetische Beeinträchtigungen einher, die durch herausnehmbaren Zahnersatz nur unzureichend kompensiert werden können (Micheelis 1997; Atchison und 
Dolan 1990; Steele et al. 2004). Viele von Zahnverlust betroffene Patienten leiden unter einem beeinträchtigten Selbstbewusstsein und fühlen sich bei alltäglichen sozialen Aktivitäten gehemmt; ebenso fällt es ihnen schwer das veränderte Erscheinungsbild zu akzeptieren (Davis et al. 2000). In einer Studie zum Einfluss von Zahnverlust und Alter auf die MLQ fanden Steele et al. (2004) heraus, dass Personen mit unter 17 Zähnen (Großbritannien) bzw. unter 21 Zähnen (Australien) die schlechtesten MLQ-Werte aufweisen (operationalisiert über den Fragebogen „14-Item Oral Health Impact Profile OHIP-14“, s. unten). Die besten MLQ-Werte erhalten Personen mit über 25 Zähnen. Bessere MLQ-Werte mit steigendem Alter lassen sich dadurch erklären, dass die Bedeutung der Mundgesundheit im Alter abnimmt, wohingegen Allgemeinerkrankungen und Einschränkungen in der Mobilität und im Intellekt zunehmen (Müller und von Steinbüchel 2005; Steele et al. 2004).

Das verbreitetste Instrument zur Befragung des Patienten nach seiner mundgesundheitsbezogenen Lebensqualität ist das Oral Health Impact Profile (OHIP) (John 2005; Slade und Spencer 1994). Entwickelt wurde das OHIP von Slade und Spencer 1994 in Australien und ist seitdem in zahlreiche Sprachen übersetzt worden. Das OHIP ist ein national sowie international verbreitetes und methodisch anspruchsvolles Instrument, das orale Erkrankungen in unterschiedlichen Ebenen einschließlich ihrer funktionellen, psychologischen und psychosozialen Auswirkungen erfasst. Die Validität wurde mittlerweile in zahlreichen nationalen und internationalen klinischen Studien, wie auch epidemiologischen Studien belegt (Kachkachishvili 2013; Liu et al. 2012; John und Micheelis 2003; McGrath und Bedi 2001; Micheelis und Schiffner 2006; Ng und Leung 2006).

Die englische Originalversion enthält 49 Items, die entsprechend des Modells von Locker (1988) sieben Subskalen zugeordnet sind:
1) Funktionelle Einschränkungen
2) Schmerzen
3) Psychisches Unwohlsein / Unbehagen
4) Physische Beeinträchtigung
5) Psychische Beeinträchtigung
6) Soziale Beeinträchtigung
7) Benachteiligung / Behinderung

2002 haben John et al. eine deutsche Version entwickelt. Diese enthält vier zusätzliche, auf die deutsche Bevölkerung abgestimmte Items, die keiner Subskala angehören. Auf Grundlage der vierten deutschen Mundgesundheitsstudie (DMS IV) wurden Normwerte für 
Deutschland entwickelt (John und Micheelis 2004; Micheelis und Schiffner 2006; John et al. 2003).

Neben der Originalversion gibt es Kurzversionen mit 21, 14 und fünf Items (John, et al., 2006), wobei die 14 Items umfassende Kurzversion, aufgrund internationaler Kompatibilität, die favorisierte ist. Die deutsche Kurzversion (OHIP-G14) (John et al. 2004) mit 14 Items basiert dabei auf der englischsprachigen Kurzversion von Slade (1997). Diese enthält jeweils zwei Fragen aus jeder Subskala und zusätzlich zwei Fragen zur allgemeinen Gesundheit und zur Mundgesundheit. Validität, Reliabilität und Änderungssensitivität der Kurzversion unterscheiden sich kaum von der Langversion (John et al. 2004).

\subsection{Forschungsergebnisse zum Zusammenhang von Parodontitis und Lebensqualität}

Seitdem die Lebensqualität in den Fokus medizinischer Interventionen gerückt ist, werden die Auswirkungen verschiedener Erkrankungen auf diese untersucht. Zunächst standen vor allem die Auswirkungen schwerer und terminaler Erkrankungen (wie z. B. Krebs) auf die Lebensqualität im Vordergrund (Bullinger et al. 2000). Zunehmend werden zwar auch die Folgen nicht direkt lebensbedrohliche Erkrankungen erforscht. Bis jetzt wurden die Auswirkungen des parodontalen Zustands auf die mundgesundheitsbezogene Lebensqualität (MLQ) allerdings nur wenig untersucht.

Für die Diagnose einer Parodontitis gibt es bereits in ihrem Frühstadium diverse klinische Untersuchungsmethoden. Die klinische Praxis zeigt jedoch häufig, dass Patienten mit einer Parodontitis selten aufgrund selbst wahrgenommener parodontaler Probleme eine Behandlung wünschen. Deshalb wird allgemein die Parodontitis als eine „stille“ Erkrankung angesehen, die bis zu einem gewissen Schweregrad eher unbemerkt von dem Patienten fortschreitet. Nach Wolf et al. (2012) sind sich Patienten keiner Parodontitis bewusst, solange sie sich voll kaufähig fühlen.

Ohrn und Jönsson (2012) entdeckten keine Korrelation zwischen verschiedenen Parodontitisvariablen und der MLQ. Auch die deutsche Mundgesundheitsstudie IV (2006) fand in der Allgemeinbevölkerung keine signifikanten Auswirkungen unterschiedlicher Parodontitisschweregrade auf die MLQ. Dieses Ergebnis erlaubt jedoch nicht die Interpretation, dass eine Parodontitis generell keinen Einfluss auf die Lebensqualität hat (Micheelis und Schiffner 2006). Ng und Leung (2006) fanden signifikante Zusammenhänge zwischen dem parodontalen Zustand und der allgemeinen Lebensqualität. Auch Shah und Kumar (2011) fanden Assoziationen zwischen Parodontitis und Lebensqualität. Ebenso Bernabé und Marcenes (2010): Sie werteten die Ergebnisse einer großen Untersuchung der britischen Allgemeinbevölkerung aus und fanden eine Assoziation zwischen 
Parodontitis und schlechterer Lebensqualität. Zudem erkannten sie eine schrittweise Verschlechterung der MLQ mit steigender Anzahl von Zähnen mit tiefen Taschen und Attachmentverlust. López und Baelum (2007) untersuchten Jugendliche und entdeckten signifikante Assoziationen von Attachmentverlust und nekrotisierender ulzerierender Gingivitis (NUG) auf die MLQ.

Darüber hinaus zeigen Studien mit behandlungssuchenden Patienten sehr wohl starke Einschränkungen der MLQ (Cunha-Cruz et al. 2007; Needleman et al. 2004). Needleman et al. (2004) berichten aus einer Untersuchung in ihrer parodontologischen Privatklinik, dass die Effekte einer Parodontitis auf die MLQ beträchtlich sind und Patienten negative Auswirkungen auf physischer, sozialer und psychologischer Ebene der Lebensqualität erleben. Nichttherapierte Neupatienten hatten darüber hinaus eine schlechtere MLQ als bereits therapierte Patienten. Ähnliche Ergebnisse hatten Saito et al. (2010), bei denen über die Hälfte der Parodontitispatienten, die für Therapie bzw. Diagnostik gekommen waren, ihre MLQ als schlecht beurteilten. Schmerzen, Einschränkungen beim Kauen und Beißen wie auch die Funktion der Zähne auf psychologischer Ebene waren die kompromittierten Domänen der MLQ. Somit kann nicht nur eine generalisierte Parodontitis, sondern auch schon eine lokalisierte Parodontitis Auswirkungen auf die MLQ haben (Bernabé und Marcenes 2010).

Es kann daher geschlussfolgert werden, dass eine negative Korrelation zwischen der Ausbreitung und dem Schweregrad der Parodontitis und der MLQ besteht (Bernabé und Marcenes 2010; Ng und Leung 2006; Cunha-Cruz et al. 2007; Needleman et al. 2004). Eine schwere chronische Parodontitis hat signifikant größere Auswirkungen auf die MLQ als eine Gingivitis und/oder leichte Parodontitis (Al Habashneh et al. 2012). Patienten mit generalisierten Formen der chronischen Parodontitis bemerken häufiger Symptome wie Zahnbeweglichkeit und unschönen Papillenverlust (Cunha-Cruz et al. 2007). Zudem sind subjektiv für den Patienten weitere wahrnehmbare Symptome Rötung, Schwellung und Blutung der Gingiva, Veränderungen der Zahnstellung, zurückgehendes Zahnfleisch, Mundgeruch und Zahnschmerzen ( $\mathrm{Ng}$ und Leung 2006; Cunha-Cruz et al. 2007; Needleman et al. 2004). Cunha-Cruz et al. (2007) und Shah und Kumar (2011) stellen deshalb in Frage, dass die Parodontitis eine „stille“ Erkrankung ist.

Shanbhag et al. (2012) kommen in ihrer Literaturübersicht zu dem Schluss, dass die Mehrheit der Studien eine Verbesserung der MLQ nach erfolgter Parodontitis-Therapie aufzeigt. Das bedeutet, dass eine Therapie also das Potenzial hat, die Wahrnehmung der Mundgesundheit zu verbessern und subjektiv dem Patienten Vorteile bietet (Shah und Kumar 2011; Saito et al. 2010). Zusätzlich zeigen Gerritsen et al. (2010) in ihrer Review, dass es eine ausreichend starke Evidenz gibt, dass auch der Zahnverlust als Folge der parodontalen Destruktion die MLQ beeinträchtigen kann. Dabei ist nicht nur die Anzahl der 
verlorenen Zähne, sondern auch ihre Lokalisation und Verteilung von Bedeutung. Auch Ohrn und Jönsson (2012) sehen nach Therapie und nötigen Extraktionen eine signifikante Korrelation zwischen der Anzahl der Zähne und MLQ. Schließlich zeigte sich auch in der deutschen Mundgesundheitsstudie DMS IV (2006) ein Zusammenhang von MLQ und Zahnverlust. Allerdings ergab sich eine Verschlechterung der MLQ erst bei einer hohen Anzahl fehlender Zähne (Micheelis und Schiffner 2006).

Zusammenfassend lässt sich festhalten, dass sich die aktuelle Studienlage als nicht einheitlich darstellt. Es fehlt an einer gesamtheitlichen Untersuchung des Zusammenhangs von MLQ und LQ sowie weiteren Einflussfaktoren und Parodontitis unter Verwendung unterschiedlicher Instrumente und somit Konzeptualisierungen von MLQ und Parodontitis. 


\section{$3 \quad$ Patienten und Methoden}

\subsection{Studientyp und -design}

Bei dieser Untersuchung handelt es sich um eine monozentrische praxisbasierte klinische Querschnittsstudie zur Feststellung bzw. Validierung eines geeigneten Studienprotokolls zur Evaluation der Zusammenhänge von parodontaler Behandlungsbedürftigkeit und mundgesundheitsbezogener Lebensqualität. Die Einschätzung der parodontalen Behandlungsbedürftigkeit des jeweiligen Patienten basierte auf einer Reihe von zahnärztlichen Befunddaten. Zur Erfassung der individuellen Lebensqualität kam eine spezifisch für diese Studie zusammengestellte Auswahl an Fragebögen zum Einsatz.

Die primäre Aufgabe der Pilotstudie lag in der Überprüfung der Tauglichkeit der Untersuchungshypothesen, der Bestimmung erfolgversprechender Studienparameter sowie der Machbarkeitsprüfung für eine anschließende großangelegte klinische Multicenter-Studie. In der Pilotstudie sollten 5-10\% des für die Hauptuntersuchung geplanten Stichprobenumfangs von 1000 Patienten in einem möglichst leicht zugänglichen Feld untersucht werden. Die Datenerhebung für die Pilotstudie fand zwischen April 2012 und Mai 2013 in der Zahnarztpraxis, in der die Untersuchungsleiterin als Zahnärztin tätig war, statt.

Die Pilotstudie wurde als Kooperationsprojekt zwischen der Poliklinik für Präventive Zahnmedizin, Parodontologie und Kariologie im Zentrum Zahn-, Mund- und Kieferheilkunde (Prof. Dr. med. dent. Rainer Mausberg) und dem Institut für Medizinische Psychologie und Medizinische Soziologie (Prof. Dr. Nicole von Steinbüchel) der Universitätsmedizin Göttingen der Georg-August-Universität geplant und durchgeführt. Zudem war Dr. Wolfgang Micheelis aus dem Institut der deutschen Zahnärzte (IDZ) in Köln an der Entwicklung dieser Studie beteiligt. Die Besonderheit dieser Studie liegt in dem komplexen Umfang, in dem die Auswirkungen einer parodontalen Behandlungsbedürftigkeit auf die Lebensqualität auf physischer, psychischer sowie sozialer Ebene so erstmalig in der Praxis untersucht worden sind.

Die Erlaubnis zur Durchführung dieser Studie wurde bei der Ethik-Kommission der GeorgAugust-Universität Göttingen beantragt und unter der Antragsnummer 20/08/11 genehmigt. Die Patienten wurden mündlich und schriftlich über die freiwillige Teilnahme, die Zielsetzungen der Studie und die vertrauliche, pseudonymisierte Behandlung der erhobenen Daten aufgeklärt. Zusätzlich wurde von allen Befragten eine schriftliche Einverständniserklärung zur Studienteilnahme unterzeichnet (s. 7.1.3 Patienteneinwilligung). 


\subsection{Gewinnung der Patientenstichprobe}

Die Auswahl der Patienten erfolgte zufällig während der zahnärztlichen Routineuntersuchung in der zahnärztlichen Praxis von den Zahnärzten Magdalene und Christoph Schoenitz in Bielefeld. In die Untersuchung wurden nur Patienten einbezogen, die ein konservierend und prothetisch mit festsitzendem Zahnersatz (Kronen und/oder Brücken) suffizient versorgtes Gebiss hatten.

Im Weiteren wurden für die Aufnahme von Patienten in die Studie folgende Einschlusskriterien festgelegt (alle Kriterien mussten erfüllt sein):

- Patienten, die zur zahnärztlichen Routineuntersuchung gekommen sind

- Patienten mit konservierend und prothetisch suffizient versorgtem Gebiss

- mindestens 20 vorhandene Zähne

- Alter > 35 Jahre

- gutes deutsches Sprachverständnis und -reproduktion

- freiwillige Teilnahme laut Einwilligungserklärung

Patienten, die eines der folgenden Ausschlusskriterien erfültten, wurden nicht in die Studie aufgenommen:

- herausnehmbarer Zahnersatz und Implantatversorgungen

- Lückengebiss (mehr als 8 fehlende Zähne)

- vorangegangene Parodontalbehandlung vor weniger als einem Jahr

- immunsupprimierte Patienten und organtransplantierte Patienten

- Hepatitis A, B, C oder TBC, HIV

- Niereninsuffizienz

- Anfalls- oder Nervenleiden oder anderen psychiatrischen Erkrankungen

- bedingte Einschränkung des Bewegungsapparates

- Suchtkrankheiten

- terminale Erkrankungen

- vorliegende Schwangerschaft (auf Nachfrage)

- erforderliche Endokarditisprophylaxe

Die Patienten wurden entsprechend über den in der zahnärztlichen Untersuchung erfassten parodontalen Screening Index (PSI) und der bestimmten parodontalen Behandlungsbedürftigkeit in zwei Gruppen eingeteilt: 
- Gruppe A: Patienten ohne parodontale Behandlungsbedürftigkeit (PSI 0-2) PSI-Werte von 0-2 in allen Sextanten bzw. maximal in zwei Sextanten höhere PSIWerte

- Gruppe B: Patienten mit parodontaler Behandlungsbedürftigkeit (PSI 3-4)

PSI von 3-4 in mindestens 3 Sextanten, davon jeweils einmal im Ober- und im Unterkiefer

Als Zielgröße wurden 25-50 Patienten je Gruppe angestrebt. Eine Vorselektion der Untersuchungsteilnehmer fand entsprechend den Einschluss- und Ausschlusskriterien durch Einsicht in die Patientenkartei statt.

\subsection{Kalibrierung}

In Bezug auf die Anwendung der objektiven Kriterien im Rahmen der zahnärztlichen Befunderhebung erfolgte vor Untersuchungsbeginn eine individuelle Kalibrierung der klinischen Untersucherin (Zahnärztin), um eine exakte Reproduzierbarkeit der Messergebnisse zu gewährleisten. Hierzu hat die Untersucherin zweimal an zwei verschiedenen Untersuchungstagen 10 Freiwillige zahnärztlich befundet und den PSI erhoben. Die Übereinstimmung lag bei über $90 \%$ und damit galt die Untersucherin als kalibriert.

\subsection{Studienplanung}

Im Rahmen der klinischen Untersuchung wurden ein ausführlicher zahnärztlicher Befund sowie der Parodontale Screening-Index (PSI) erhoben und zusammen mit der Angabe über bereits durchgeführte Parodontitistherapien in einem zahnärztlichen Untersuchungsbogen erfasst (siehe Anlage 7.1.4).

Im Anschluss erfolgte die Erhebung der Daten der Parodontitis-Checkliste nach $\mathrm{Ng}$ und Leung (2006) und des Oral Health Impact Profile (OHIP-G14) (John et al. 2004) in Interviewform zwischen der Untersucherin und dem Patienten. Die restlichen sieben Fragebögen zur Erfassung von Gesundheitszustand, Lebensqualität, negativer Emotionen sowie soziodemografischer Daten wurden durch die Patienten in einem separaten Raum selbstständig ausgefüllt (s. Tabelle 1). Für etwaige Fragen seitens der Patienten stand die Untersucherin zur Verfügung. 
Tabelle 1: Erhebungszweck und Durchführungsmodalität der sieben Fragebögen der eingesetzten Fragebogenbatterie

\begin{tabular}{|c|c|c|}
\hline Fragebogen & Erhebungszweck & Durchführung \\
\hline (1) Parodontitis-Checkliste & $\begin{array}{lr}\text { Erfassung spezifische } \\
\text { Symptome einer Parodontitis }\end{array}$ & $\begin{array}{l}\text { Interview durch } \\
\text { Untersuchungsleiter }\end{array}$ \\
\hline $\begin{array}{l}\text { (2) Oral Health Impact Profile } \\
\text { (OHIP-G 14) }\end{array}$ & $\begin{array}{l}\text { Erfassung der } \\
\text { mundgesundheitsbezogenen } \\
\text { Lebensqualität (MLQ) }\end{array}$ & $\begin{array}{l}\text { Interview durch } \\
\text { Untersuchungsleiter }\end{array}$ \\
\hline $\begin{array}{l}\text { (3) Fragebogen Ihr Allgemeiner } \\
\text { Gesundheitszustand }\end{array}$ & $\begin{array}{l}\text { Erfassung des objektiven } \\
\text { Gesundheitszustands }\end{array}$ & Ausgefüllt durch Patient \\
\hline $\begin{array}{l}\text { (4) Short-Form Health Survey } \\
\text { (SF-36) }\end{array}$ & $\begin{array}{l}\text { Erfassung des subjektiven } \\
\text { Gesundheitszustands und } \\
\text { krankheitsbedingter } \\
\text { Belastungen } \\
\text { (Lebensqualität) }\end{array}$ & Ausgefüllt durch Patient \\
\hline $\begin{array}{l}\text { (5) World Health Organization } \\
\text { Quality of Life Scale } \\
\text { (WHOQOL-BREF) }\end{array}$ & $\begin{array}{l}\text { Erfassung der eigenen } \\
\text { Lebensqualität }\end{array}$ & Ausgefüllt durch Patient \\
\hline $\begin{array}{l}\text { (6) Hospital Anxiety and } \\
\text { Depression Scale (HADS-D) }\end{array}$ & $\begin{array}{l}\text { Erfassung von Angst und } \\
\text { Depression }\end{array}$ & Ausgefüllt durch Patient \\
\hline $\begin{array}{l}\text { (7) Soziodemografischer } \\
\text { Fragebogen }\end{array}$ & $\begin{array}{l}\text { Erfassung } \\
\text { soziodemografischer Daten }\end{array}$ & Ausgefüllt durch Patient \\
\hline
\end{tabular}

Jedem Patienten wurde eine Patientennummer für seine Daten zugewiesen und damit die Datenblätter pseudonymisiert. Die persönlichen Daten der Patienten wurden vertraulich behandelt und nur zu Studienzwecken verwendet.

\subsection{Studiendurchführung}

\section{Zahnärztlicher Befund}

Zur Bestimmung des Sanierungs- und Versorgungsgrades wurde ein ausführlicher zahnärztlicher Befund aufgenommen. Bei der Befundaufnahme erfolgte eine systematische klinische Untersuchung aller Zähne mit Spiegel und Sonde unter standardisierten Bedingungen in einem zahnärztlichen Behandlungsstuhl. Dabei wurden suffiziente und insuffiziente Füllungen, Zahnersatz und kariöse Läsionen sowie fehlende Zähne dokumentiert. Waren Zahnlücken und/oder Zahnhartsubstanzdefekte mit festsitzendem Zahnersatz versorgt, erfolgte ebenfalls eine Beurteilung nach Suffizienz bzw. Insuffizienz. Insuffizient versorgte Patienten und Patienten mit herausnehmbarem Zahnersatz oder stark reduziertem Lückengebiss (mehr als 8 fehlenden Zähnen) wurden für die Untersuchung nicht berücksichtigt (siehe Ausschlusskriterien). 
Die Beurteilungskriterien für suffizient bzw. insuffizient waren wie folgt definiert:

\section{Suffizient:}

- kariesfreies Gebiss / Restauration

- guter Randschluss der Restaurationen, d. h. suffiziente marginale / zirkuläre Adaptation

- korrekte okklusale Gestaltung und Okklusion der Restaurationen

- funktionell korrekte Approximalkontakte der Restaurationen

\section{Insuffizient:}

- überstehender oder inkompletter Randschluss (Randspalt), d. h. insuffiziente marginale / zirkuläre Adaptation

- Sekundärkaries

- nicht korrekte okklusale Gestaltung der restaurativen Kaufläche und NonOkklusion

- fehlender Approximalkontakt

- großflächige Keramikabplatzung

Aus dem zahnärztlichen Befund wurde der Kariesindex Decayed-Missing-Filled Teeth Index (DMF-T) abgeleitet. Dazu wurde die Summe gebildet aus der Anzahl vorhandener Zähne ( $\mathbf{T}$ =teeth) mit konservierender Versorgung (Füllungen) und prothetischer Kronenversorgung ( $\mathbf{F}=$ filled), sowie fehlender $(\mathbf{M}=$ missed) und kariöser bzw. zerstörter Zähne ( $\mathbf{D}=$ decayed). Der DMF-T-Index kann einen Maximalwert von 28 annehmen (ohne Berücksichtigung der Weisheitszähne). Ein hoher DMF-T-Wert steht für eine hohe Karieserfahrung.

\subsubsection{Der Parodontale Screening-Index (PSI)}

Zur Zuordnung in eine der beiden Gruppen A: PSI 1-2 und B: PSI 3-4 wurde der Parodontale Screening-Index (PSI) erhoben.

Der Parodontale Screening-Index (PSI) dient der schnellen Feststellung einer parodontalen Behandlungsbedürftigkeit. Er wurde von der American Academy of Periodontology (AAP) und der American Dental Association (ADA) entwickelt und beruht auf dem modifizierten Community Periodontal Index of Treatment Needs (CPITN) der WHO (World Health Organization - WHO 1948; Ainamo et al. 1982). Zur Bestimmung des individuellen PSI wird das Gebiss in Sextanten eingeteilt. An jedem Zahn fand eine Sechs-Punkt-Messung statt (mesiobukkal, bukkal, distobukkal, mesiolingual, lingual, distolingual). Dabei wurde für jeden einzelnen Messpunkt ein Codewert zwischen 0 - 4 ermittelt (s. Tabelle 2). 
Tabelle 2: Bewertungsgrade des PSI

\begin{tabular}{|c|c|c|c|}
\hline $\begin{array}{l}\text { PSI- } \\
\text { Code }\end{array}$ & Befund & $\begin{array}{l}\text { Diagnose / mögliche } \\
\text { vorliegende } \\
\text { Erkrankung }\end{array}$ & Therapiekonsequenz \\
\hline 0 & $\begin{array}{ll}\text { - } & \text { Farbige Markierung vollständig } \\
& \text { sichtbar } \\
\text { - } & \text { Kein Bluten auf Sondieren } \\
\text { - } & \text { Kein Zahnstein } \\
\text { - } & \text { Keine marginalen Irritationen }\end{array}$ & $\begin{array}{l}\text { Gesunde } \\
\text { Parodontalverhältnisse }\end{array}$ & Kein Behandlungsbedarf \\
\hline 1 & $\begin{array}{ll}\text { - } & \text { Farbige Markierung vollständig } \\
& \text { sichtbar } \\
\text { - } & \text { Blutung auf Sondierung } \\
\text { - } & \text { Kein Zahnstein } \\
\text { - } & \text { Keine marginalen Irritationen }\end{array}$ & Gingivitis & Mundhygieneinstruktionen \\
\hline 2 & $\begin{array}{l}\text { Farbige Markierung vollständig } \\
\text { sichtbar } \\
\text { - Zahnstein und/oder iatrogene } \\
\text { marginale Irritationen }\end{array}$ & Gingivitis & $\begin{array}{l}\text { Zusätzlich zu den } \\
\text { Mundhygieneinstruktionen } \\
\text { eine professionelle } \\
\text { Zahnreinigung und } \\
\text { Zahnsteinentfernung }\end{array}$ \\
\hline 3 & $\begin{array}{l}\text { Farbige Markierung nur noch } \\
\text { teilweise sichtbar } \\
\text { (Sondierungstiefe } \\
3,5-5,5 \mathrm{~mm} \text { ) }\end{array}$ & $\begin{array}{l}\text { Mittelschwere bis } \\
\text { schwere Parodontitis }\end{array}$ & $\begin{array}{l}\text { Systematische } \\
\text { Parodontalbehandlung mit } \\
\text { ausführlicher Diagnostik } \\
\text { und Therapie }\end{array}$ \\
\hline 4 & $\begin{array}{l}\text { - Farbige Markierung nicht mehr } \\
\text { sichtbar } \\
\text { (Sondierungstiefe über } \\
5,5 \mathrm{~mm} \text { ) }\end{array}$ & $\begin{array}{l}\text { Mittelschwere bis } \\
\text { schwere Parodontitis }\end{array}$ & $\begin{array}{l}\text { Systematische } \\
\text { Parodontalbehandlung mit } \\
\text { ausführlicher Diagnostik } \\
\text { und Therapie }\end{array}$ \\
\hline
\end{tabular}

Der jeweils schlechteste Einzelmesswert war der Referenzwert für den gesamten Sextanten und wurde in eine dafür vorgesehene Sechsfeldertafel übertragen. Wurde an einer Stelle der Codewert 4 gemessen, konnte direkt zum nächsten Sextanten übergegangen werden. Pseudotaschen distal der 7er wurden nicht berücksichtigt. Der Index wurde mit der sogenannten „WHO-Sonde“ erhoben (Kugel von 0,5 mm Durchmesser, am Sondenende schwarze Markierung zwischen 3,5 und 5,5 mm) (Abbildung 4).

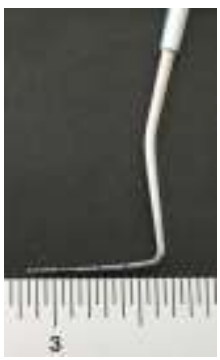

Abbildung 4: WHO-Sonde mit Skalierung (eigenes Bild) 
Das Sondieren erfolgte möglichst schmerzfrei für den Patienten. Die Sonde wurde dazu leicht zwischen Zahn und Zahnfleisch eingeführt. Die aufgewendete Kraft sollte dabei zwischen 15 und $26 \mathrm{~g}$ liegen.

Neben den Sondierungstiefen wurden Zahnstein, Blutungen und defekte Restaurationsränder befundet. Die Sondierungstiefen wurden an der Farbmarkierung der Sonde abgelesen. Das kugelförmige Sondenende diente dem Austasten von subgingivalem Zahnstein und überstehenden Restaurationsrändern. Blutungen wurden nach einer Zeitspanne von etwa 10 Sekunden nach Sondierung sextantenweise bewertet. Für weitere klinische Auffälligkeiten wie Furkationsbefall, Rezessionen, Zahnlockerung und mukogingivale Probleme wurde dem entsprechenden Codewert das Kürzel „ * “ (in Worten: Sternchen) beigefügt.

\subsection{Fragebögen zur Erhebung des Gesundheits- zustands und der Lebensqualität der Teilnehmer}

Zur Erfassung der Patientendaten wurde ein Unterlagenpaket der folgenden Fragebogen in Papierform zusammengestellt (s. Kapitel 7.1 Formulare).

\subsubsection{Parodontitis-Checkliste (Pcheck-12)}

Die Parodontitis-Checkliste (Pcheck-12) wurde in Anlehnung an die Arbeit von $\mathrm{Ng}$ und Leung entwickelt (2006). Mit Hilfe von 16 Fragen im dichotomen Ja-Nein-Format wurden spezifische Symptome einer möglichen bzw. vorliegenden Parodontitis der letzten 12 Monate erhoben. Die ersten 12 Fragen der Parodontitis-Checkliste beziehen sich auf Parodontitis-spezifische Symptome (wie z. B. selbst festgestellte und/oder wahrgenommene geschwollene, schmerzende, zurückgehende Gingiva, Zahnlockerung und/oder -wanderung, Mundgeruch und/oder Zahnschmerzen) und werden additiv im Pcheck-12-Summenscore zusammengefasst. Die letzten vier Fragen beziehen sich auf Vorbehandlungen und Untersuchungen des Patienten.

\subsubsection{Oral Health Impact Profile (OHIP-G14)}

Mit Hilfe der deutschen Kurzversion des Oral Health Impact Profile (OHIP-G14) von John et al. (2004) wurde die mundgesundheitsbezogene Lebensqualität erfasst. Die ersten beiden zusätzlichen Fragen geben Aufschluss über die Einschätzung des eigenen allgemeinen Gesundheitszustands und des Mundgesundheitszustands. In den weiteren 14 Fragen wird die mundgesundheitsbezogene Lebensqualität anhand der Ausprägung einzelner Indikatoren im Zeitraum der vergangenen vier Wochen erhoben. Die Antwortmöglichkeiten der Mehrstufenskala umfassen folgende Abstufungen: „nie“, „kaum“, „ab und zu“, „oft“ und „sehr oft". Für die Auswertung wird den Antworten ein Punktwert zwischen „nie“ $=0$ und 
„sehr oft“ = 4 zugeordnet. Die Punktwerte der 14 Fragen werden zu dem Wert „MLQ“ summiert. Dieser Wert kann zwischen 0 (wenn alle 14 Fragen mit „nie“ beantwortet werden) und 56 (wenn alle Fragen mit „sehr oft“ beantwortet werden) liegen. Es gilt also: Je höher der MLQ-Wert, desto schlechter die mundgesundheitsbezogene Lebensqualität.

\subsubsection{Allgemeiner Gesundheitsfragebogen}

Beim Fragebogen zum allgemeinen Gesundheitszustand handelt es sich um ein kurzes Screening zur Erfassung spezifischer medizinischer Symptomatiken (Von Steinbüchel et al. 2006). Es werden 30 aktuell vorliegende physische und psychische Erkrankungen im Selbstbeurteilungsverfahren erfasst. Die Item-Beantwortung erfolgt im dichotomen Ja-NeinFormat und wurde von den Patienten selbst ausgefüllt.

\subsubsection{Short-Form Health Survey (SF-36)}

Der Short-Form Health Survey (SF-36) ist ein Instrument zur Messung der gesundheitsbezogenen Lebensqualität für den krankheitsübergreifenden Einsatz (Ware und Sherbourne 1992; Bullinger und Kirchberger 1998). Er wurde im Rahmen des 1960 in Amerika begonnenen Forschungsprojekts, der sogenannten Medical Outcome Study (MOS), aus einem 100 Items umfassenden Instrument (NORC) entwickelt und beschreibt den individuellen Gesundheitszustand sowie die krankheitsbedingten Belastungen (und somit die Lebensqualität). Die Validität und Reliabilität dieses international etablierten Standardinstruments ist kulturübergreifend umfangreich überprüft (Bullinger 1995; Apolone und Mosconi 1998; Ellert und Bellach 1999; Ellert und Kurth 2013; Bjorner et al. 1998; Hsiung et al. 2005).

Der SF-36 umfasst 36 Items, die die nachfolgenden acht Dimensionen subjektiver Gesundheit erfassen:

(1) Körperliche Funktionsfähigkeit

(2) Körperliche Rollenfunktion

(3) Körperliche Schmerzen

(4) Allgemeine Gesundheitswahrnehmung

(5) Emotionale Rollenfunktion

(6) Vitalität

(7) Psychisches Wohlbefinden

(8) Soziale Funktionsfähigkeit

Die acht Dimensionen sind den zwei Grunddimensionen subjektiver Gesundheit „Körperliche Gesundheit (KSK)“ und „Psychische Gesundheit (PSK)“ zugeordnet (s. Abbildung 5). 


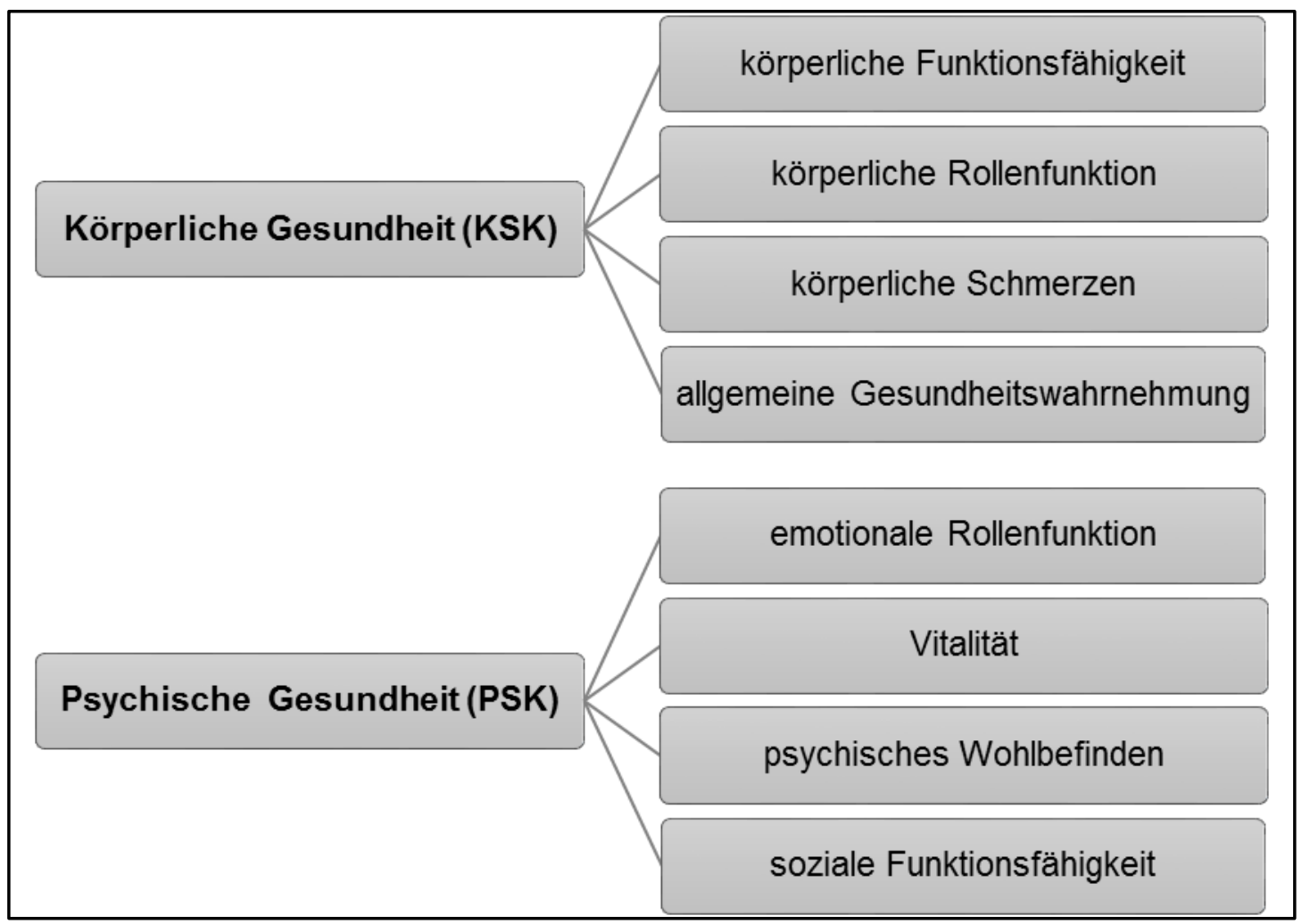

Abbildung 5: Zuordnung in die zwei Grunddimensionen: körperliche und psychische Gesundheit

Die Antwortkategorien variieren vom dichotomen Ja-Nein-Format bis hin zu sechsstufigen Antwortskalen. Der Fragebogen wurde in dieser Studie als Selbstbeantwortungsversion für den Zeitraum von vier Wochen eingesetzt.

\subsubsection{World Health Organization Quality of Life Scale (WHOQOL- BREF)}

Der World Health Organization Quality of Life Scale (WHOQOL-BREF) ist ein Instrument zur Erfassung der allgemeinen subjektiven Lebensqualität (Angermeyer et al. 2000). Grundlage ist die WHO-Definition für die Lebensqualität (WHOQOL Group 1995). Bei dem WHOQOL-BREF handelt es sich um die die Kurzversion des 100 Items umfassenden WHOQOL-100 wobei über 26 Items die folgenden vier Domänen der Lebensqualitätseinschätzung der Person erhoben werden (WHOQOL Group 1998):

(1) Physisches Wohlbefinden,

(2) Psychisches Wohlbefinden,

(3) Soziale Beziehungen und

(4) Umwelt 
Der WHOQOL-BREF ist ein international etabliertes und gut validiertes Instrument, das eine weltweite Vergleichbarkeit von Lebensqualitätsdaten ermöglicht (Hsiung et al. 2005; Min et al. 2002; Noerholm et al. 2004; Ohaeri et al. 2004; Skevington et al. 2004; Trompenaars et al. 2005).

\subsubsection{Hospital Anxiety and Depression Scale - Deutsche Version (HADS-D)}

Die deutsche Version der Hospital Anxiety and Depression Scale (HADS-D) von Herrman, Buss und Snaith (1995) ist ein kurzer Selbstbeurteilungsbogen zur Erfassung von Angst und Depressivität, der gezielt für den Einsatz bei Personen in somatisch-medizinischen Einrichtungen entwickelt wurde (Herrmann-Lingen et al. 2011). Die HADS ist ein valides Instrument zur Diagnostik von und zur Bestimmung der Symptomschwere von Angststörungen und Depressionen bei somatischen sowie bei psychiatrischen Patienten; aber auch bei Patienten in der Erstversorgung und bei Personen aus der Allgemeinbevölkerung (Bjelland et al. 2002; Herrmann-Lingen et al. 2011). Die HADS-D umfasst 14 Items, gegliedert in zwei Subskalen, die jeweils mit sieben Items Angst- und Depressivitätssymptome messen. Die Beantwortung der Items erfolgte auf einer vierstufigen Likert-Skala. Der Wertebereich liegt zwischen 0 und 21, wobei höhere Werte Auffälligkeiten kennzeichnen (<7 unauffällig, 8-10 grenzwertig, >11 auffällig).

\subsection{Soziodemografischer Fragebogen}

Mit dem soziodemografischen Fragebogen von von Steinbüchel wurden allgemeine soziodemografische und spezifische Daten im Selbstbeurteilungsverfahren erfasst (Von Steinbüchel et al. 2006). Mittels 11 Items mit vorgegebenen Antwortkategorien wurden folgende Personendaten in den folgenden sieben Bereichen erhoben:

(1) Ausbildung

(2) Beruf

(3) Lebenssituation

(4) Autonomie

(5) Freizeit und Sozialleben

(6) Genussmittelkonsum

(7) wichtige Lebensereignisse 


\subsection{Statistische Auswertung}

Die erhobenen klinischen Daten und Ergebnisse der Fragebögen wurden für die statistische Auswertung in das Tabellenkalkulationsprogramm Microsoft ECXEL 2010 (Microsoft Corporation, Redmond, USA) übertragen. Mit diesem Programm erfolgte die Berechnung der Mittelwerte, Standardabweichungen, Minima und Maxima. Die inferenzstatistische Auswertung wurde in Zusammenarbeit mit dem Institut für medizinische Statistik und dem Institut für medizinische Psychologie und medizinische Soziologie der Universitätsmedizin Göttingen über die Statistiksoftware IBM SPSS Version 22 (International Business Machines Corporation [IBM], Armonk, USA) und SAS Version 9.3 (SAS Institute, Cary, USA) durchgeführt.

Das Signifikanzniveau wurde mit 5\% festgelegt. Der Signifikanzwert und die verschiedenen inferenzstatistische Testverfahren zur Feststellung von Gruppenunterschieden (ChiQuadrat Test, Fisher-Yates Test, t-Test, Mann-Whitney U-Test) und Zusammenhängen (Korrelation nach Spearman) werden an den jeweiligen Textstellen erwähnt. Die beiden Gruppen A (PSI 1-2) und B (PSI 3-4) wurden anhand der Analysevariablen OHIP-G14, Parodontitis-Checkliste, HADS-Angst, HADS-Depressivität, den beiden Grunddimensionen des SF-36 körperliche Gesundheit und psychische Gesundheit, wie auch den drei Domänen des WHOQOL-BREF physisches Wohlbefinden, psychisches Wohlbefinden, soziale Beziehungen und Umwelt deskriptiv sowie inferenzstatistisch miteinander verglichen. 


\section{Ergebnisse}

\subsection{Patientenkollektiv}

An der Pilotstudie nahmen insgesamt 49 Patienten teil. Die Patienten wurden entsprechend ihres PSI-Wertes in zwei Gruppen eingeteilt.

Unter den Patienten befanden sich 34 Frauen (69\%) und 15 Männer (31\%). Das Durchschnittsalter aller Patienten lag bei $49 \pm 9,7$ Jahren. Raucher waren mit $23 \%$ in Gruppe A und 13\% in Gruppe B in der Minderheit. In beiden Gruppen zeigten Familienstand und Ausbildungsgrad eine ausgewogene Verteilung. 26 Patienten hatten keine parodontale Behandlungsbedürftigkeit (Gruppe A: PSI 1-2) und 23 Patienten wiesen eine parodontale Behandlungsbedürftigkeit auf (Gruppe B: PSI 3-4).

Tabelle 3 gibt entsprechend der Gruppenaufteilung nach dem PSI-Wert einen Überblick über die Patientencharakteristika Alter, Geschlecht, Rauchgewohnheiten, Familienstand und Ausbildungsgrad.

Tabelle 3: Patientencharakteristika in beiden Vergleichsgruppen

\begin{tabular}{|c|c|c|c|}
\hline Parameter & Ausprägung & $\begin{array}{c}\text { Gruppe A (PSI 1-2) } \\
n=26\end{array}$ & $\begin{array}{c}\text { Gruppe B (PSI 3-4) } \\
n=23\end{array}$ \\
\hline \multirow[t]{2}{*}{ Alter } & $\mathrm{MW} \pm \mathrm{SD}$ & $46 \pm 8$ & $52 \pm 10.5$ \\
\hline & Min/Max & $35 / 63$ & $37 / 81$ \\
\hline \multirow{2}{*}{$\begin{array}{l}\text { Geschlecht } \\
n(\%)\end{array}$} & Männlich & $6(23)$ & $9(39)$ \\
\hline & Weiblich & $20(77)$ & $14(61)$ \\
\hline \multirow{3}{*}{$\begin{array}{l}\text { Rauchgewohnheiten } \\
\mathrm{n}(\%)\end{array}$} & Nichtraucher & $14(54)$ & $18(78)$ \\
\hline & Raucher & $6(23)$ & $3(13)$ \\
\hline & Ehemaliger Raucher & $6(23)$ & $2(9)$ \\
\hline \multirow{5}{*}{$\begin{array}{l}\text { Familienstand } \\
\mathrm{n}(\%)\end{array}$} & Single & $4(15)$ & $2(9)$ \\
\hline & Verheiratet & $19(73)$ & $18(78)$ \\
\hline & Liiert & $2(8)$ & $1(4)$ \\
\hline & Getrennt/geschieden & $1(4)$ & $1(4)$ \\
\hline & Verwitwet & $0(0)$ & $1(4)$ \\
\hline \multirow{5}{*}{$\begin{array}{l}\text { Ausbildungsgrad } \\
\mathrm{n}(\%)\end{array}$} & Grundschule & $0(0)$ & $1(4)$ \\
\hline & Sekundarstufe & $4(15)$ & $3(13)$ \\
\hline & Lehre & $11(42)$ & $12(52)$ \\
\hline & Fachschulabschluss & $6(23)$ & $5(22)$ \\
\hline & $\begin{array}{l}\text { Fachhochschul- } \\
\text { /Universitätsabschluss }\end{array}$ & $5(19)$ & $2(9)$ \\
\hline
\end{tabular}


Ein signifikanter Zusammenhang zwischen Gruppenzugehörigkeit und Geschlecht besteht nicht $(p=0,352$, Fisher-Yates Test). Jedoch unterscheiden sich die beiden Gruppen in Bezug auf das durchschnittliche Patientenalter ( $p=0,03$, t-Test). Für die Eigenschaften „Raucher" $(p=0,19)$, Familienstand $(p=0,76)$ und Ausbildungsgrad ( $p=0,67$, jeweils ChiQuadrat-Test nach Pearson) liegen wiederum keine signifikanten Unterschiede vor.

\subsection{Zahnärztlicher Befund}

\subsubsection{Decayed-Missing-Filled Teeth Index (DMF-T)}

Der mittlere DMF-T-Wert aller Probanden betrug 16,2 $\pm 4,4$. In Gruppe A war ein mittlerer DMF-T-Wert von 14,9 \pm 4,4 feststellbar, in Gruppe B von 17,7 $\pm 3,9$. Aufgrund der Auswahlkriterien gab es keine Patienten mit kariösen Läsionen ( $D-T=0)$. In Gruppe A fehlten maximal 6 Zähne; in Gruppe B maximal 8 Zähne. Durchschnittlich waren in Gruppe A 13,2 $\pm 4,2$ Zähne gefüllt oder mit festsitzendem Zahnersatz (Kronen) versehen. In Gruppe B lag der Mittelwert des F-T bei 14,5 $\pm 3,8$ (s. Tabelle 4).

Tabelle 4: Mittelwerte, Standardabweichung, minimale und maximale Werte der Studiengruppen in Bezug auf den dentalen Befund

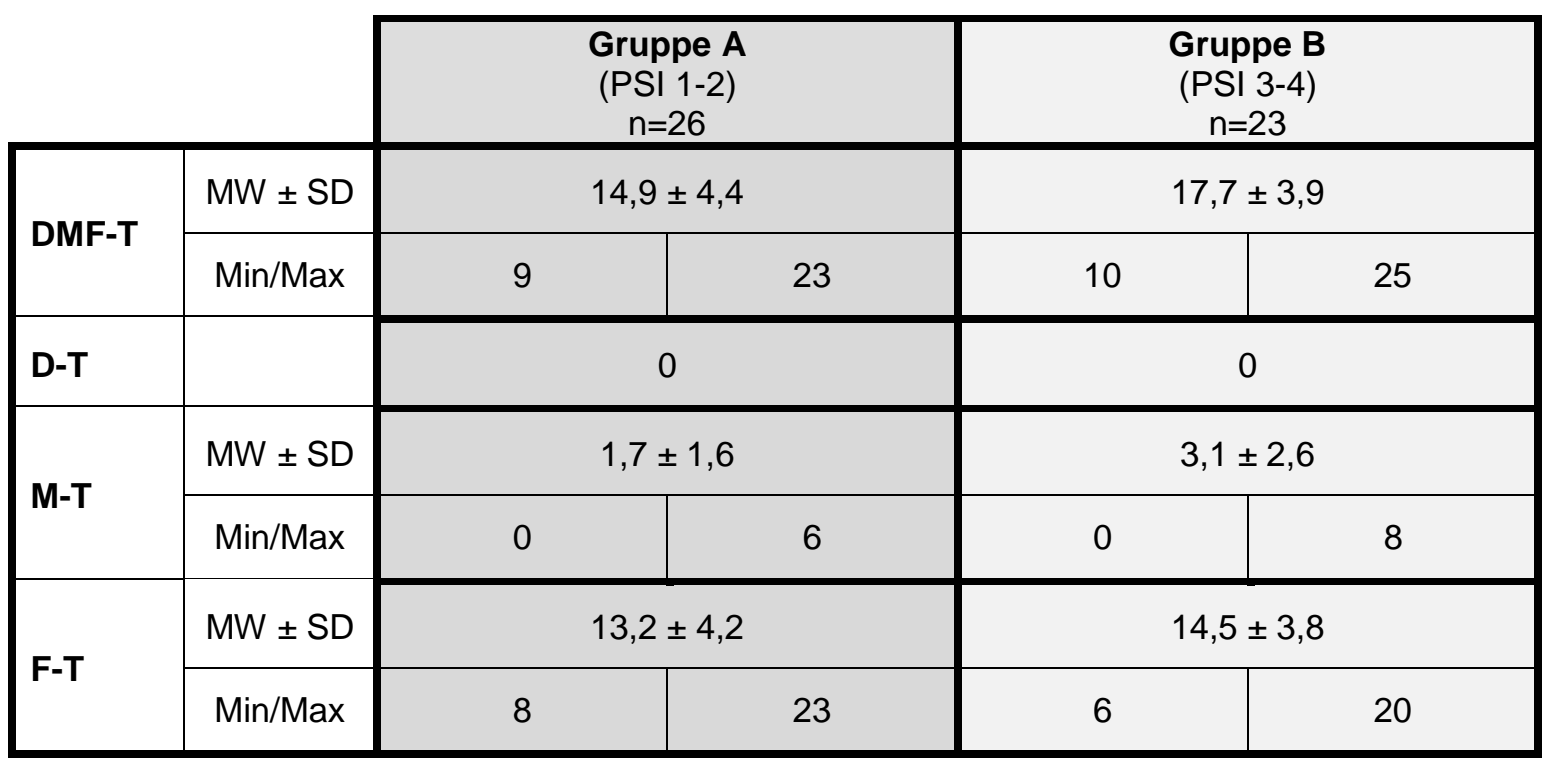

Zwischen den beiden Gruppen ergeben sich beim DMF-T und für M-T signifikante Differenzen (beide $p=0,02$, t-Test). Für F-T liegt keine Signifikanz vor ( $p=0,27, t-T e s t)$. 


\subsubsection{Parodontaler Screening-Index (PSI)}

Insgesamt wies kein Patient einen maximalen PSI-Wert von 0 auf ( 0 in allen Sextanten). Ausschließlich ein Patient hatte einen maximalen PSI-Wert von 1, 23 Patienten von maximal 2. Zwei Patienten zeigten einen maximalen PSI-Wert von 3. Dieser war jeweils nur in einem Sextanten vorhanden, so dass diese zwei Patienten entsprechend der Kriterien für die Gruppeneinteilung der Gruppe A („ohne parodontale Behandlungsbedürftigkeit“) zugeordnet wurden. 12 Patienten hatten (in mehreren Sextanten) einen maximalen PSIWert von 3 und 11 Patienten einen von 4 und entsprachen den weiteren Kriterien für die Gruppeneinteilung in die Gruppe B (,mit parodontaler Behandlungsbedürftigkeit“) (s. Tabelle 5).

Tabelle 5: Absolute Häufigkeit der maximalen PSI-Werte in den beiden Studiengruppen

\begin{tabular}{|l|c|c|}
\cline { 2 - 3 } \multicolumn{1}{c|}{} & $\begin{array}{c}\text { Gruppe A (PSI 1-2) } \\
\mathrm{n}=26 \\
\mathrm{n}(\%)\end{array}$ & $\begin{array}{c}\text { Gruppe B (PSI 3-4) } \\
\mathrm{n}=23 \\
\mathrm{n}(\%)\end{array}$ \\
\hline PSI 0 & $0(0)$ & $0(0)$ \\
\hline PSI 1 & $1(4)$ & $0(0)$ \\
\hline PSI 2 & $23(88)$ & $0(0)$ \\
\hline PSI 3 & $2(8)$ & $12(52)$ \\
\hline PSI 4 & $0(0)$ & $11(48)$ \\
\hline
\end{tabular}

Für Gruppe A (PSI 1-2) zeigte sich für die vier Sextanten im Seitenzahnbereich (I, III, IV, VI) eine ähnliche Verteilung der PSI-Werte. Im Sextant II (Oberkieferfrontzähne) gab es die wenigsten Blutungen auf Sondierung $(P S I=0)$. Im Sextant $V$ wiesen alle, bis auf einen Patienten, Zahnstein auf (Abbildung 6).

Für Gruppe B (PSI 3-4) war in den vier Sextanten im Seitenzahnbereich (I, III, IV, VI) die größte parodontale Behandlungsbedürftigkeit festzustellen. Der PSI-Wert 4 war im Oberkiefer-Seitenzahnbereich am häufigsten vertreten. Die Frontzahnsextanten (II, V) zeigten in ca. einem Drittel der Fälle eine parodontale Behandlungsbedürftigkeit. In dieser Gruppe gab es keinen Patienten, der in einem Sextanten keine Blutung auf Sondierung aufwies (PSI=0) (s. Abbildung 7). 


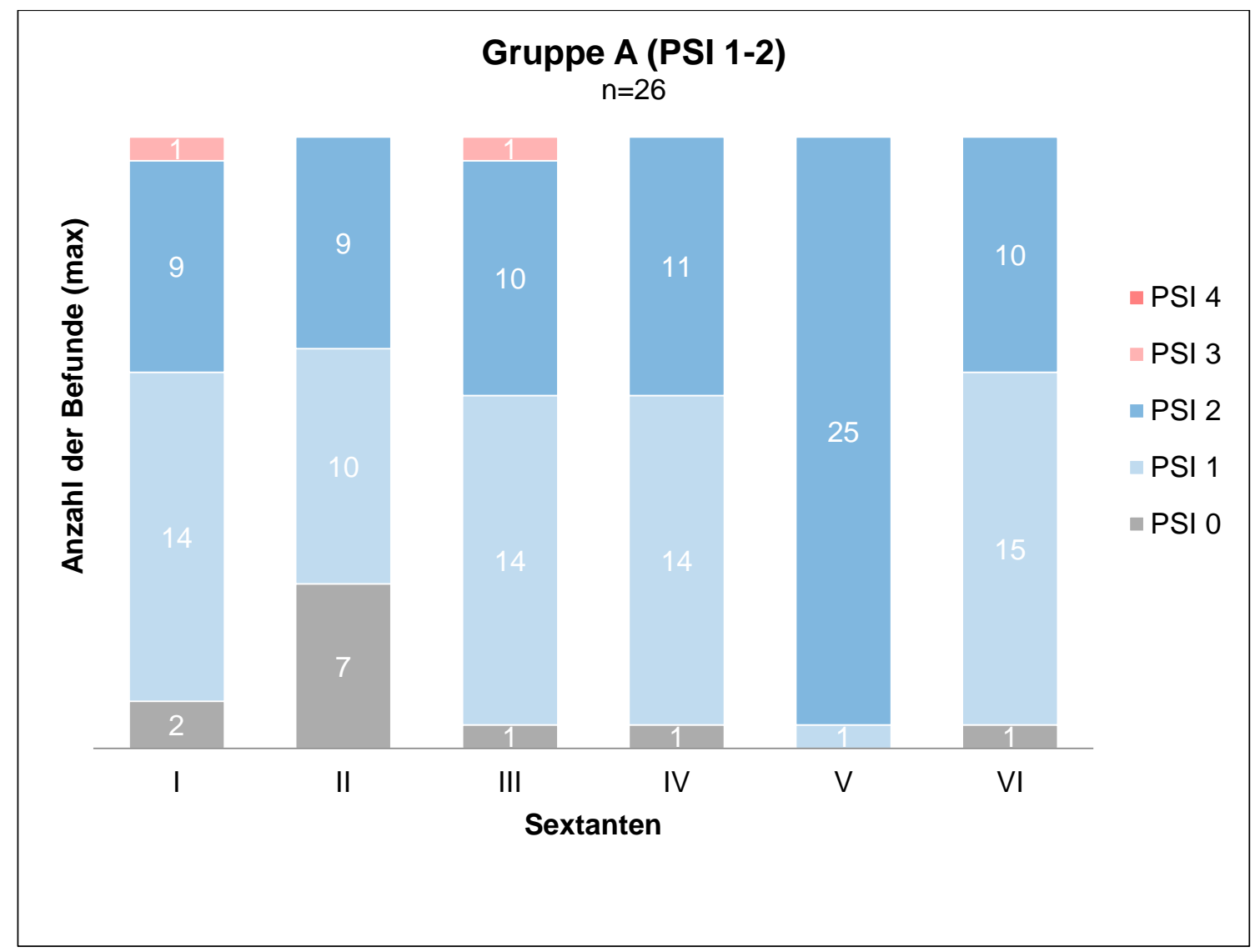

Abbildung 6: Verteilung der PSI-Werte der einzelnen Sextanten in Gruppe A (PSI 1-2)

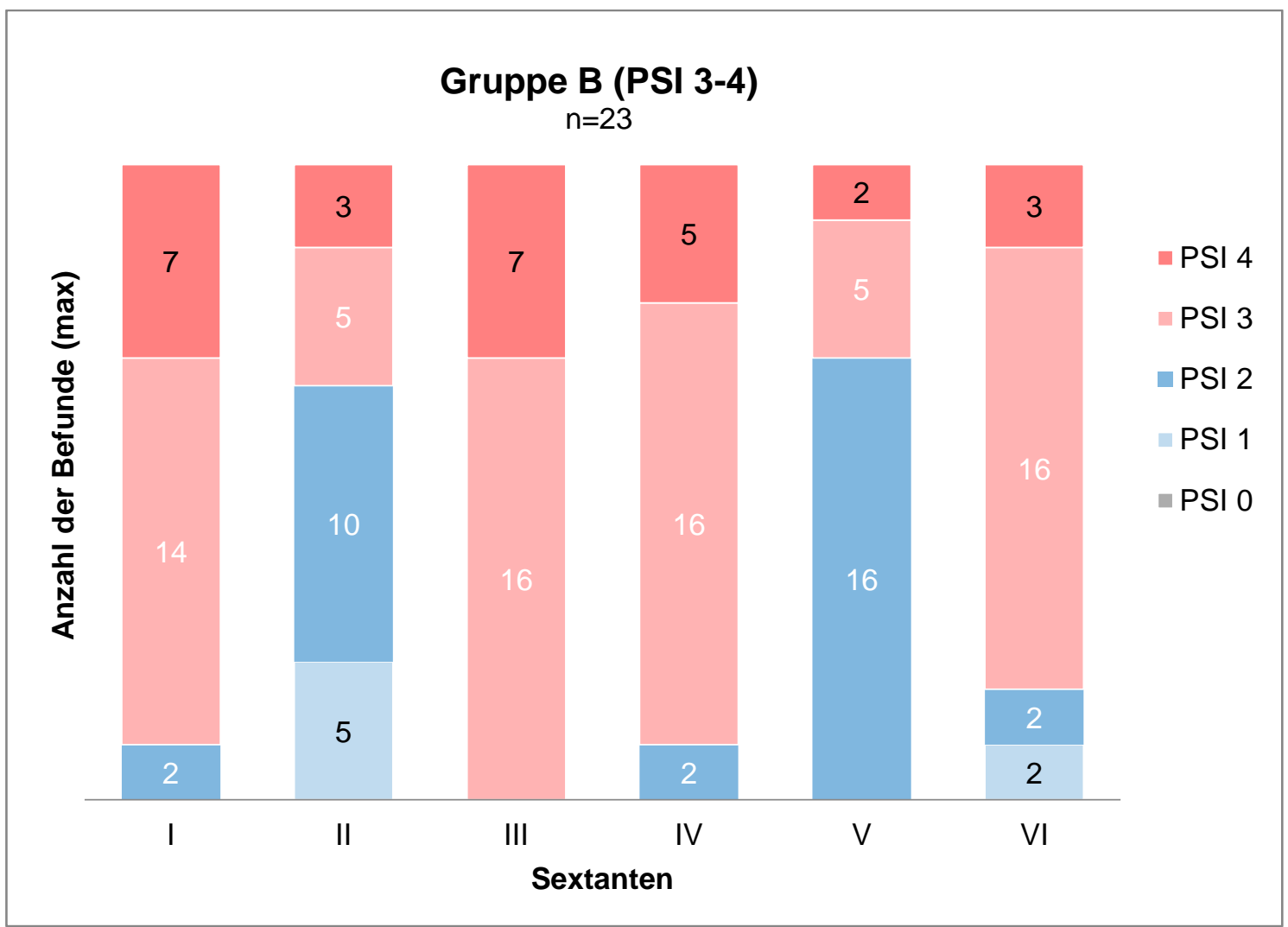

Abbildung 7: Verteilung der PSI-Werte der einzelnen Sextanten in Gruppe B (PSI 3-4) 


\subsection{Befragung zu Gesundheitszustand und Lebensqualität}

\subsubsection{Parodontitis-Checkliste (Pcheck-12)}

Die nachfolgende Tabelle 6 zeigt Parodontitis-spezifische Symptome in ihrer absoluten Häufigkeit. Die am häufigsten genannten Symptome in beiden Gruppen waren empfindliches Zahnfleisch, Zahnfleischbluten, zurückgehendes Zahnfleisch und Zahnüberempfindlichkeit. Empfindliches Zahnfleisch trat mit 43\% häufiger in Gruppe B, gegenüber 19\% in Gruppe A, auf. Somit bestand eine Tendenz ( $p=0,06$, Fisher-Yates Test). Die Verteilung der anderen Symptome war in beiden Gruppen ausgeglichen und nicht signifikant unterschiedlich ( $p>0,05$; $s$. Tabelle 6).

Tabelle 6: Verteilung der einzelnen Parodontitis-spezifischen Symptome in beiden Gruppen

\begin{tabular}{|l|c|c|}
\cline { 2 - 3 } \multicolumn{1}{c|}{} & $\begin{array}{c}\text { Gruppe A (PSI 1-2) } \\
\mathrm{n}=26 \\
\mathrm{n}(\%)\end{array}$ & $\begin{array}{c}\text { Gruppe B (PSI 3-4) } \\
\mathrm{n}=23 \\
\mathrm{n}(\%)\end{array}$ \\
\hline Zahnfleischschwellung & $3(12)$ & $4(17)$ \\
\hline Schmerzendes Zahnfleisch & $2(8)$ & $4(17)$ \\
\hline Empfindliches Zahnfleisch & $5(19)$ & $10(43)$ \\
\hline Zahnfleischbluten & $9(35)$ & $9(39)$ \\
\hline Zurückgehendes Zahnfleisch & $10(38)$ & $11(48)$ \\
\hline Zahnüberempfindlichkeit & $12(46)$ & $14(61)$ \\
\hline Zahnlockerung & $0(0)$ & $2(9)$ \\
\hline Zahnwanderung & $0(0)$ & $2(9)$ \\
\hline Veränderung des Bisses & $1(4)$ & $0(0)$ \\
\hline Mundgeruch & $2(8)$ & $3(13)$ \\
\hline Schlechter Geschmack & $3(12)$ & $1(4)$ \\
\hline Zahnschmerzen & $3(12)$ & $2(9)$ \\
\hline
\end{tabular}

Gruppe B wies eine leichte Tendenz zu einer höheren Gesamtzahl Parodontitis-spezifischer Symptome auf. Dieses spiegelte sich im Pcheck-12-Summenscore in den unterschiedlichen Maximalwerten (Gruppe A: 5; Gruppe B: 8), Mittelwerten und Standardabweichungen (Gruppe A: 1,92 1,72; Gruppe B: 2,7 $\pm 2,16$ ) und dem Median (Gruppe A: 1; Gruppe B: 2) beider Gruppen wieder. Ein statistisch signifikanter Unterschied zwischen den beiden Gruppen besteht jedoch nicht ( $p=0,171$, t-Test).

Gemäß den Auswahlkriterien wurde bei keinem der in die Studie aufgenommenen Patienten in den letzten 12 Monaten eine Parodontitis-Behandlung durchgeführt. Die große Mehrzahl der Patienten kam regelmäßig zur Kontrolluntersuchung (96\% der Patienten aus Gruppe A und $91 \%$ der Patienten aus Gruppe B). In den letzten zwölf Monaten war ein Drittel der Patienten aus Gruppe A (31\%) zur beschwerdebedingten Kontrolluntersuchung 
in der Praxis, in Gruppe B waren es vier Patienten (17\%). 81\% der Patienten aus Gruppe A lassen regelmäßig eine professionelle Zahnreinigung durchführen, in Gruppe B sind dies hingegen nur $65 \%$ (s. Tabelle 7).

Tabelle 7: Verteilung der zahnärztlichen Vorsorge in beiden Gruppen

\begin{tabular}{|l|c|c|}
\cline { 2 - 3 } \multicolumn{1}{c|}{} & $\begin{array}{c}\text { Gruppe A (PSI 1-2) } \\
\mathrm{n}=26 \\
\mathrm{n}(\%)\end{array}$ & $\begin{array}{c}\text { Gruppe B (PSI 3-4) } \\
\mathrm{n}=23 \\
\mathrm{n}(\%)\end{array}$ \\
\hline $\begin{array}{l}\text { Vorab Paradontitis- } \\
\text { Behandlung }\end{array}$ & 0 & 0 \\
\hline $\begin{array}{l}\text { Regelmäßige } \\
\text { Kontrolluntersuchung }\end{array}$ & $25(96 \%)$ & $21(91 \%)$ \\
\hline $\begin{array}{l}\text { Beschwerdebedingte } \\
\text { Kontrolluntersuchung }\end{array}$ & $8(31 \%)$ & $4(17 \%)$ \\
\hline $\begin{array}{l}\text { Regelmäßige professionelle } \\
\text { Zahnreinigung }\end{array}$ & $21(81 \%)$ & $15(65 \%)$ \\
\hline
\end{tabular}

\subsubsection{Oral Health Impact Profile (OHIP-G14)}

Die Mehrzahl der Patienten bewertete ihren allgemeinen Gesundheitszustand und Mundgesundheitszustand als gut bis ausgezeichnet (Allgemeiner Gesundheitszustand Gruppe A: 92\%, Gruppe B: 91\%; Mundgesundheitszustand Gruppe A: 81\%, Gruppe B: 91\%). Tendenziell wurde in Gruppe A der allgemeine Gesundheitszustand $(2,62 \pm 0,8)$ von den Patienten etwas besser eingeschätzt als ihr Mundgesundheitszustand $(2,23 \pm 0,77)$. In Gruppe B waren die angegebenen Werte zum allgemeinen und Mund-Gesundheitszustand ausgeglichen $(2,26 \pm 0,62$ bzw. 2,22 $\pm 0,6)$. Der Unterschied in der Einschätzung des eigenen Mundgesundheitszustands ist zwischen den Gruppen nicht signifikant $(p=0,41$, Mann-Whitney-U Test gerichtet). Allerdings beurteilen die Patienten der Gruppe A ihren allgemeinen Gesundheitszustand signifikant besser als Patienten der Gruppe B ( $p=0,05$, Mann-Whitney-U Test gerichtet). Kein Patient beurteilte seinen allgemeinen und MundGesundheitszustand als schlecht (s. Abbildung 8). 


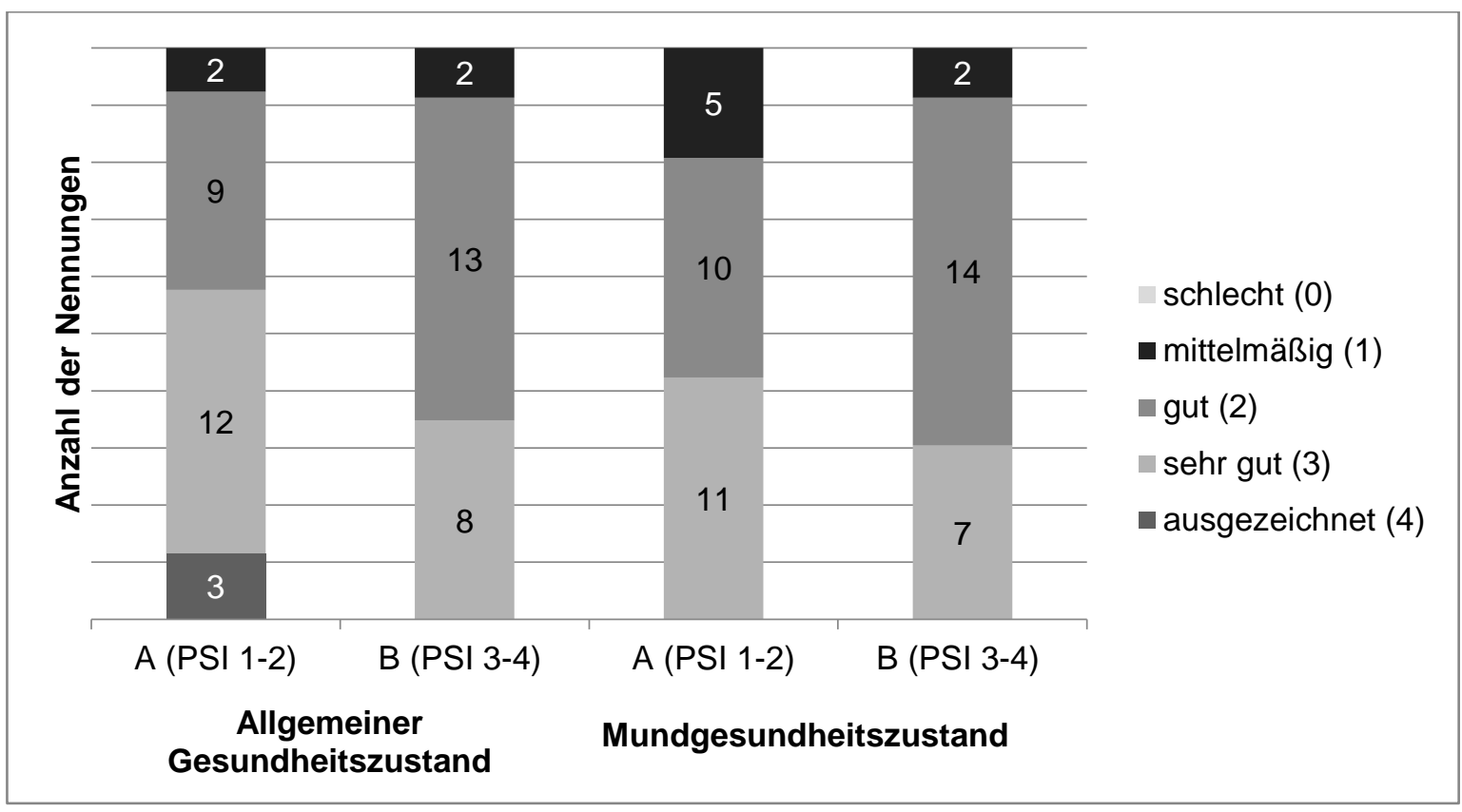

Abbildung 8: Graphische Darstellung zur Einschätzung der Patienten ihres allgemeinen und Mundgesundheitszustands in beiden Gruppen

Die Hälfte der Fragen des OHIP-G14 zur mundgesundheitsbezogenen Lebensqualität wurde in beiden Studiengruppen mit „nie“ beantwortet (s. Tabelle 8); dies betrifft die Subskalen „Funktionelle Einschränkungen“ (Frage 1 „Sprachschwierigkeiten“ und 2 „Beeinträchtigung des Geschmacksinns“), „Physische Beeinträchtigung“ (Frage 6 „Unterbrechung von Mahlzeiten“ und 12 „Unbefriedigende Ernährung“), „soziale Beeinträchtigung" (Frage 8 „Soziale Reizbarkeit“ und 9 "Schwierigkeiten bei Alltagsbeschäftigungen“) und „Benachteiligung/Behinderung“ (Frage 10 „Unfähigkeit, etwas zu tun“). Leichte Einschränkungen wurden auf den Subskalen „Psychische Beeinträchtigung“ (Frage 4 „Schwierigkeiten zu entspannen“ und 11 "Gefühl der Verlegenheit"), „Psychisches Unwohlsein“ (Frage 5 "Gefühl der Anspannung“ und 14 „Gefühl der Unsicherheit“) und „Schmerzen“ (Frage 7 „Unangenehmes Essgefühl“ und 13 „Schmerzen“) berichtet. Hier wurde als Antwortalternative vorzugsweise „ab und zu“ und „kaum“ gewählt. Auf der Subskala „Benachteiligung/Behinderung“ wurde lediglich die Frage 3 („Leben weniger Zufriedenstellend“) mit „ab und zu“ und „kaum“ beantwortet.

Zwölf Patienten (46\%) aus Gruppe A und zehn Patienten (43\%) aus Gruppe B haben alle Fragen mit „nie“ beantwortet. Das Maximum der Gesamtsummenwerte lag in Gruppe A bei 9 und in Gruppe B bei 11 von einem maximal erzielbaren Gesamtsummenwert von 56. 
Tabelle 8: Profil der Antworten des OHIP-G14 absolut und in Prozent in Gruppe A (PSI 1-2) und Gruppe B (PSI 3-4)

\begin{tabular}{|c|c|c|c|c|c|c|c|c|c|c|}
\hline \multirow{2}{*}{$\begin{array}{l}\text { Hatten Sie im vergangenen Monat aufgrund von Problemen mit } \\
\text { Ihren Zähnen, im Mundbereich oder mit ihrem Zahnersatz... }\end{array}$} & \multicolumn{2}{|c|}{$\begin{array}{c}\text { sehr oft (4) } \\
n(\%)\end{array}$} & \multicolumn{2}{|c|}{$\begin{array}{l}\text { oft (3) } \\
n(\%)\end{array}$} & \multicolumn{2}{|c|}{$\begin{array}{c}\text { ab und zu (2) } \\
n(\%)\end{array}$} & \multicolumn{2}{|c|}{$\begin{array}{c}\text { kaum (1) } \\
\mathrm{n}(\%)\end{array}$} & \multicolumn{2}{|c|}{$\begin{array}{c}\text { nie (0) } \\
\mathrm{n}(\%)\end{array}$} \\
\hline & A & B & A & B & A & B & A & B & A & B \\
\hline 1. Schwierigkeiten bestimmte Worte auszusprechen? & - & - & - & - & $1(4)$ & - & $1(4)$ & - & $\begin{array}{l}24 \\
(96)\end{array}$ & $\begin{array}{c}23 \\
(100)\end{array}$ \\
\hline 2. das Gefühl, Ihr Geschmackssinn war beeinträchtigt? & - & - & - & - & - & - & $2(8)$ & - & $\begin{array}{l}25 \\
(96)\end{array}$ & $\begin{array}{c}23 \\
(100)\end{array}$ \\
\hline $\begin{array}{ll}\text { 3. den Eindruck, dass Ihr Leben ganz allgemein weniger } \\
\text { zufriedenstellend war? }\end{array}$ & - & - & - & - & $1(4)$ & $2(9)$ & $5(19)$ & $3(13)$ & 20 & $\begin{array}{l}18 \\
(78)\end{array}$ \\
\hline 4. Schwierigkeiten zu entspannen? & - & - & - & $1(4)$ & $3(12)$ & - & $4(15)$ & $2(9)$ & $\begin{array}{l}19 \\
(73)\end{array}$ & $\begin{array}{l}20 \\
(87)\end{array}$ \\
\hline
\end{tabular}

\begin{tabular}{|c|c|c|c|c|c|c|c|c|c|c|}
\hline \multirow{3}{*}{$\begin{array}{l}\text { Ist es im vergangenen Monat aufgrund von Problemen mit Ihren } \\
\text { Zähnen, im Mundbereich oder mit Ihrem Zahnersatz vorgekommen... } \\
5 . \text { dass Sie sich angespannt gefühlt haben? }\end{array}$} & \multicolumn{2}{|c|}{$\begin{array}{l}\text { sehr oft (4) } \\
n(\%)\end{array}$} & \multicolumn{2}{|c|}{$\begin{array}{l}\text { oft (3) } \\
n(\%)\end{array}$} & \multicolumn{2}{|c|}{$\begin{array}{l}\text { ab und zu (2) } \\
n(\%)\end{array}$} & \multicolumn{2}{|c|}{$\begin{array}{c}\operatorname{kaum}(1) \\
\mathrm{n}(\%)\end{array}$} & \multicolumn{2}{|c|}{$\begin{array}{c}\text { nie (0) } \\
\mathrm{n}(\%)\end{array}$} \\
\hline & \multicolumn{2}{|c|}{ A $\quad$ B } & \multicolumn{2}{|c|}{ A $\quad$ B } & \multicolumn{2}{|c|}{ A $\quad$ B } & \multicolumn{2}{|c|}{ A $\quad$ B } & A & B \\
\hline & - & - & - & - & $2(8)$ & - & $2(8)$ & $4(17)$ & $\begin{array}{l}22 \\
(85)\end{array}$ & $\begin{array}{c}19 \\
(83)\end{array}$ \\
\hline 6. dass Sie Ihre Mahlzeiten unterbrechen mussten? & - & - & - & - & - & - & - & $2(9)$ & $\begin{array}{c}26 \\
(100)\end{array}$ & $\begin{array}{l}21 \\
(91)\end{array}$ \\
\hline $\begin{array}{l}\text { 7. dass es Ihnen unangenehm war, bestimmte Nahrungsmittel zu } \\
\text { essen? }\end{array}$ & $1(4)$ & - & - & - & $1(4)$ & $4(17)$ & $1(4)$ & $1(4)$ & $\begin{array}{l}23 \\
(88)\end{array}$ & $\begin{array}{c}18 \\
(78)\end{array}$ \\
\hline $\begin{array}{l}\text { 8. dass Sie anderen Menschen gegenüber eher reizbar gewesen } \\
\text { sind? }\end{array}$ & - & - & - & - & - & - & $1(4)$ & $1(4)$ & $\begin{array}{l}25 \\
(96)\end{array}$ & $\begin{array}{l}22 \\
(96)\end{array}$ \\
\hline $\begin{array}{ll}\text { 9. dass es Ihnen schwergefallen ist, Ihren alltäglichen } \\
\text { Beschäftigungen nachzugehen? }\end{array}$ & - & - & - & - & - & - & - & $1(4)$ & $\begin{array}{c}26 \\
(100)\end{array}$ & $\begin{array}{l}22 \\
(96)\end{array}$ \\
\hline 10. dass Sie vollkommen unfähig waren, etwas zu tun? & - & - & - & - & - & - & - & - & $\begin{array}{c}26 \\
(100)\end{array}$ & $\begin{array}{c}23 \\
(100)\end{array}$ \\
\hline 11. dass Sie sich ein wenig verlegen gefühlt haben? & - & - & - & - & $1(4)$ & $1(4)$ & $4(15)$ & $1(4)$ & $\begin{array}{l}21 \\
(81)\end{array}$ & $\begin{array}{l}21 \\
(91)\end{array}$ \\
\hline 12. dass Ihre Ernährung unbefriedigend gewesen ist? & - & - & - & - & - & - & $1(4)$ & $3(13)$ & $\begin{array}{l}25 \\
(96)\end{array}$ & $\begin{array}{c}20 \\
(87)\end{array}$ \\
\hline \multirow[t]{2}{*}{ Hatten Sie im vergangenen Monat... } & \multicolumn{2}{|c|}{$\begin{array}{l}\text { sehr oft (4) } \\
n(\%)\end{array}$} & \multicolumn{2}{|c|}{$\begin{array}{l}\text { oft (3) } \\
n(\%)\end{array}$} & \multicolumn{2}{|c|}{$\begin{array}{l}\text { ab und zu (2) } \\
n(\%)\end{array}$} & \multicolumn{2}{|c|}{$\begin{array}{l}\text { kaum (1) } \\
n(\%)\end{array}$} & \multicolumn{2}{|c|}{$\begin{array}{c}\text { nie (0) } \\
\mathrm{n}(\%)\end{array}$} \\
\hline & A & B & A & B & A & B & A & B & A & B \\
\hline 13. Schmerzen im Mundbereich? & - & - & - & - & - & $4(17)$ & $2(8)$ & $5(22)$ & $\begin{array}{c}24 \\
(92)\end{array}$ & $\begin{array}{l}14 \\
(61)\end{array}$ \\
\hline $\begin{array}{l}\text { 14. ein Gefühl der Unsicherheit in Zusammenhang mit Ihren Zähnen, } \\
\text { Ihrem Mund oder Ihrem Zahnersatz? }\end{array}$ & - & - & $2(8)$ & - & $2(8)$ & $3(13)$ & - & $3(13)$ & $\begin{array}{l}22 \\
(85)\end{array}$ & $\begin{array}{l}17 \\
74)\end{array}$ \\
\hline
\end{tabular}




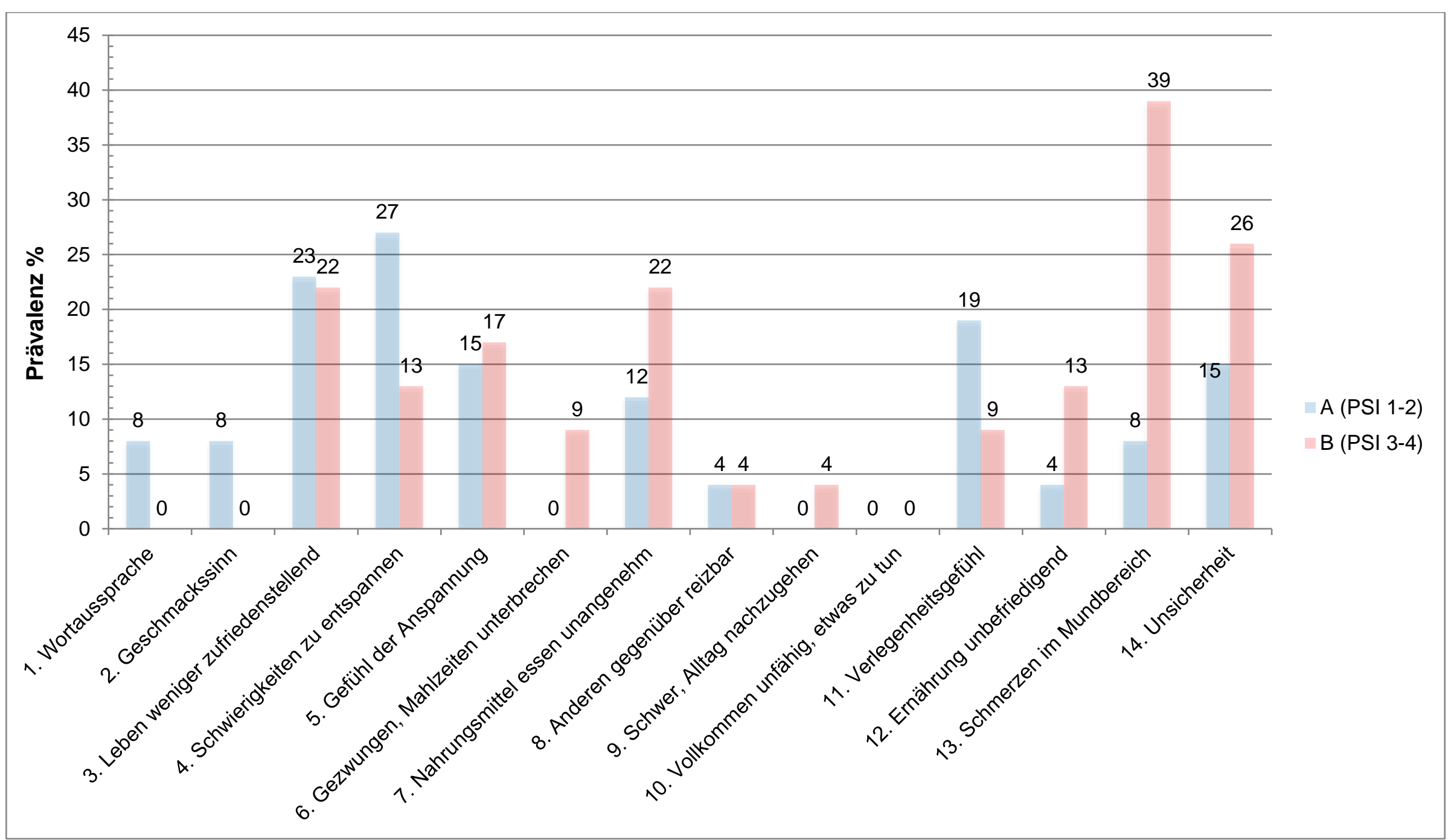

Abbildung 9: Prävalenz von generell vorhandenen Mundgesundheitsproblemen (d. h. Minimalwert von 1 „kaum“) aus dem OHIP-G14 in beiden Gruppen 
Abbildung 9 gibt die Prävalenz von generell vorhandenen Mundgesundheitsproblemen bei Berücksichtigung jeder positiven Antwort (d. h. Minimalwert von 1 „kaum“) der auf dem OHIP-G14 berichteten Beeinträchtigungen der mundgesundheitsbezogenen Lebensqualität für die beiden Gruppen wieder.

Aufgeteilt in die zwei Studiengruppen A und B lagen bei den folgenden Items die höchsten Prävalenzen vor (s. Tabelle 9):

Tabelle 9: Prävalenzwerte der berichteten Beeinträchtigungen bei Berücksichtigung jeder positiven Antwort im OHIP-G14 getrennt nach den Studiengruppen

\begin{tabular}{|l|l|}
\hline \multicolumn{1}{|c|}{$\begin{array}{c}\text { Gruppe A (PSI 1-2) } \\
\mathrm{n}=26\end{array}$} & \multicolumn{1}{c|}{$\begin{array}{c}\text { Gruppe B (PSI 3-4) } \\
\mathrm{n}=23\end{array}$} \\
\hline $\begin{array}{l}27 \% \text { „Schwierigkeiten zu entspannen“ } \\
\text { (Frage 4) }\end{array}$ & $39 \%$ „Schmerzen“ (Frage 13) \\
\hline $\begin{array}{l}23 \% \text { „Leben weniger zufriedenstellend“ } \\
\text { (Frage 3) }\end{array}$ & $26 \%$ „Gefühl der Unsicherheit“ (Frage 14) \\
\hline $9,6 \%$ „Verlegenheitsgefühl“ (Frage 11) & $\begin{array}{l}22 \% \text { „Nahrungsmittel essen unangenehm“ } \\
\text { (Frage 7) }\end{array}$ \\
\hline
\end{tabular}

In Gruppe B hatte somit die Subskala „Schmerzen“ die höchste Prävalenz. Allein in der Frage 13 („Hatten Sie im vergangenen Monat Schmerzen im Mundbereich?“) unterschieden sich die beiden Untersuchungsgruppen signifikant voneinander. Hier berichtete Gruppe B sehr signifikant über häufigere Schmerzen ( $p<0,01$, Mann-Whitney-U Test).

Die Mittelwerte der MLQ-Werte waren in beiden Gruppen ähnlich $(A: 2,12 \pm 2,58$; $B$ : $2,48 \pm 3,1$ ). Der Median beider Gruppen lag bei 1. Es besteht kein signifikanter Unterschied ( $p=0,66$, $t-T e s t)$ zwischen den beiden Gruppen hinsichtlich des MLQ-Wertes.

\subsubsection{Allgemeiner Gesundheitsfragebogen}

Aus Gruppe A gaben im Allgemeinen Gesundheitsfragebogen 92\% der Studienteilnehmer an, „gesund“ zu sein; in Gruppe B waren es 96\%. Ein signifikanter Unterschied besteht nicht ( $p=1,0$, Fisher-Yates Test 2-seitig). In Tabelle 10 sind die am häufigsten genannten Gesundheitszustände und Probleme zusammengestellt. 
Tabelle 10: Am häufigsten genannte Probleme (ab 6 Nennungen) aus dem Fragebogen „Allgemeiner Gesundheitszustand“ absolut und in Prozent für beide Studiengruppen

\begin{tabular}{|l|c|c|}
\cline { 2 - 3 } \multicolumn{1}{c|}{} & $\begin{array}{c}\text { Gruppe A (PSI 1-2) } \\
\mathrm{n}=26 \\
\mathrm{n}(\%)\end{array}$ & $\begin{array}{c}\text { Gruppe B (PSI 3-4) } \\
\mathrm{n}=23 \\
\mathrm{n}(\%)\end{array}$ \\
\hline Allergien & $6(23)$ & $6(26)$ \\
\hline Schilddrüsenprobleme & $7(27)$ & $2(9)$ \\
\hline Schlafprobleme & $3(12)$ & $9(39)$ \\
\hline Kopfschmerzen/Migräne & $6(23)$ & $6(26)$ \\
\hline Mangel an Energie & $8(31)$ & $2(9)$ \\
\hline Rückenprobleme & $10(38)$ & $9(39)$ \\
\hline
\end{tabular}

Ein signifikanter Unterschied besteht hier bei Schlafproblemen $(p=0,04$, Fisher-Yates Test 2-seitig, Anzahl der Nennungen siehe Tabelle). Weiterhin unterscheiden sich die Studiengruppen tendenziell signifikant bei der Frage nach „Hohem Blutzucker oder Diabetes“ ( $p=0,01$, Fisher-Yates Test 2-seitig, Gruppe A: keine Nennungen, Gruppe B: 3 Nennungen). In beiden Fällen schneiden Befragte aus Gruppe B schlechter ab.

\subsubsection{Short-Form Health Survey (SF-36)}

Die Einschätzung des subjektiven Gesundheitszustands und der krankheitsbedingten Belastungen anhand des SF-36 wurde auf der körperlichen als auch der psychischen Summenskala (KSK bzw. PSK) mit der deutschen Normstichprobe (Bullinger und Kirchberger, 1998) verglichen (s. Tabelle 11).

Tabelle 11: Mittelwerte, Standardabweichung, minimale und maximale Werte der KSK bzw. PSK in beiden Studiengruppen und der deutschen Normstichprobe

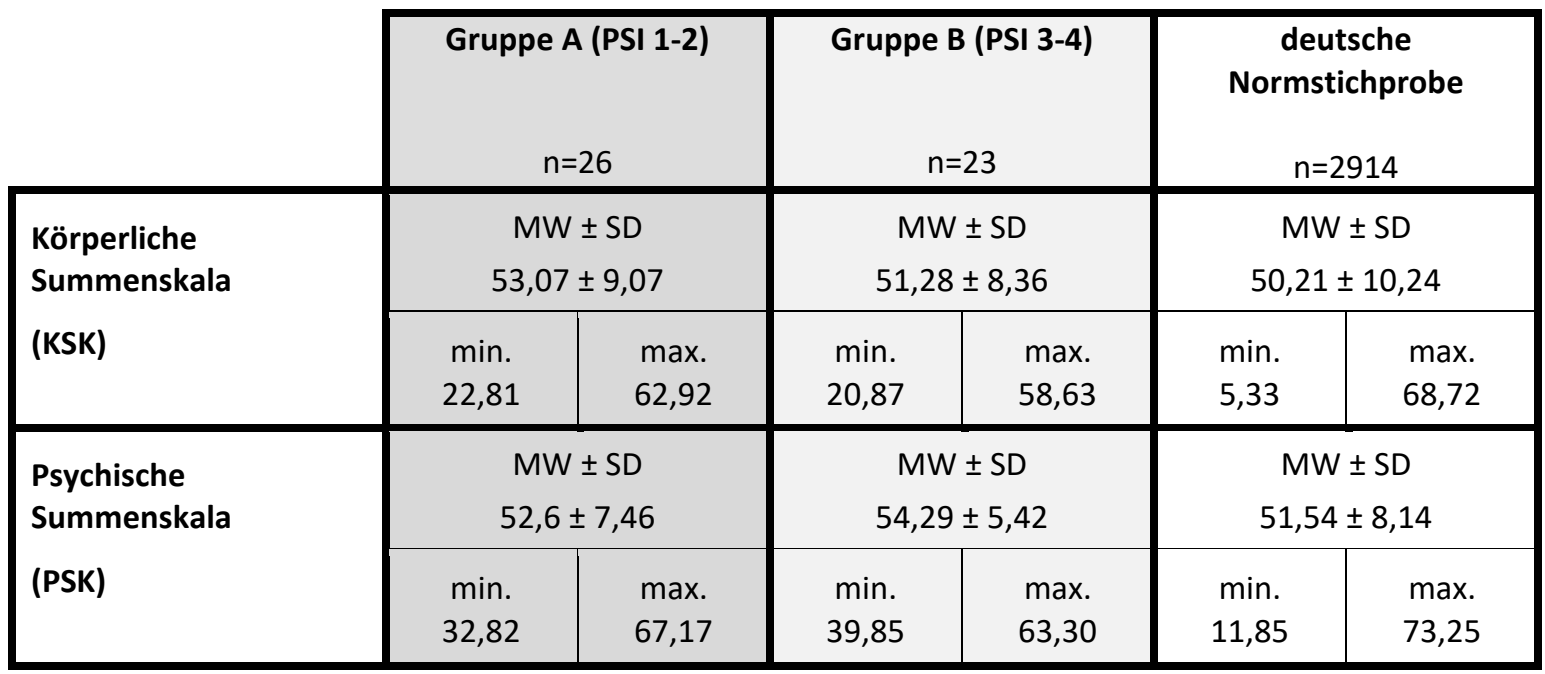


Die beiden Studiengruppen entsprachen der Norm und zeigten tendenziell einen besseren subjektiven Gesundheitszustand als die deutsche Normstichprobe. Es bestanden keine signifikanten Unterschiede zwischen den Studiengruppen, weder in den beiden Summenskalen (KSK: $p=0,49$, PSK $p=0,38$, t-Test), noch auf Ebene der Einzelitems.

\subsubsection{World Health Organization Quality of Life Scale (WHOQOL- BREF)}

Die allgemeine subjektive Lebensqualität gemessen über den WHOQOL-BREF wurde in den vier Domänen „Physisches Wohlbefinden“, „Psychisches Wohlbefinden“, „Soziale Beziehungen“ und „Umwelt“ ausgewertet (s. Tabelle 12).

Tabelle 12: Mittelwerte, Standardabweichung, minimale und maximale Werte in beiden Studiengruppen und in der Normbevölkerung in den vier Domänen des WHOQOL-BREF

\begin{tabular}{|c|c|c|c|c|c|}
\hline Domänen & \multicolumn{2}{|c|}{$\begin{array}{c}\text { Gruppe A (PSI 1-2) } \\
\qquad n=26\end{array}$} & \multicolumn{2}{|c|}{$\begin{array}{c}\text { Gruppe B (PSI 3-4) } \\
\qquad n=23\end{array}$} & $\begin{array}{c}\text { Normwerte deutsche } \\
\text { Allgemein- } \\
\text { bevölkerung } \\
n=2055\end{array}$ \\
\hline \multirow[t]{2}{*}{ Physisch } & \multicolumn{2}{|c|}{$\begin{array}{c}\mathrm{MW} \pm \mathrm{SD} \\
84,20 \pm 11,61\end{array}$} & \multicolumn{2}{|c|}{$\begin{array}{c}\mathrm{MW} \pm \mathrm{SD} \\
80,84 \pm 12,5\end{array}$} & $\mathrm{MW} \pm \mathrm{SD}$ \\
\hline & $\begin{array}{c}\min . \\
57,14\end{array}$ & $\begin{array}{c}\max . \\
100\end{array}$ & $\begin{array}{c}\min . \\
32,14\end{array}$ & $\begin{array}{l}\max . \\
92,86\end{array}$ & $76,92 \pm 17,68$ \\
\hline \multirow[t]{2}{*}{ Psychisch } & \multicolumn{2}{|c|}{$\begin{array}{c}\mathrm{MW} \pm \mathrm{SD} \\
79,5 \pm 8,75\end{array}$} & \multicolumn{2}{|c|}{$\begin{array}{c}\mathrm{MW} \pm \mathrm{SD} \\
79,17 \pm 7,39\end{array}$} & $\mathrm{MW} \pm \mathrm{SD}$ \\
\hline & $\begin{array}{c}\min . \\
58,33\end{array}$ & $\begin{array}{l}\max . \\
95,83\end{array}$ & $\begin{array}{c}\min . \\
58,33\end{array}$ & $\begin{array}{l}\max . \\
95,83\end{array}$ & $74,02 \pm 15,68$ \\
\hline \multirow[t]{2}{*}{$\begin{array}{l}\text { Soziale } \\
\text { Beziehungen }\end{array}$} & \multicolumn{2}{|c|}{$\begin{array}{c}\mathrm{MW} \pm \mathrm{SD} \\
80,33 \pm 12,93\end{array}$} & \multicolumn{2}{|c|}{$\begin{array}{c}\mathrm{MW} \pm \mathrm{SD} \\
79,17 \pm 11,31\end{array}$} & $\mathrm{MW} \pm \mathrm{SD}$ \\
\hline & $\begin{array}{c}\min . \\
50\end{array}$ & $\begin{array}{c}\max . \\
100\end{array}$ & $\begin{array}{l}\min . \\
50\end{array}$ & $\begin{array}{c}\max . \\
100\end{array}$ & $71,83 \pm 18,52$ \\
\hline \multirow[t]{2}{*}{ Umwelt } & \multicolumn{2}{|c|}{$\begin{array}{c}\mathrm{MW} \pm \mathrm{SD} \\
82,00 \pm 8,99\end{array}$} & \multicolumn{2}{|c|}{$\begin{array}{c}\mathrm{MW} \pm \mathrm{SD} \\
80,36 \pm 7,47\end{array}$} & $\mathrm{MW} \pm \mathrm{SD}$ \\
\hline & $\begin{array}{l}\min . \\
65,63\end{array}$ & $\begin{array}{c}\max . \\
100\end{array}$ & $\begin{array}{l}\min . \\
65,63\end{array}$ & $\begin{array}{l}\max . \\
90,63\end{array}$ & $70,38 \pm 14,17$ \\
\hline
\end{tabular}

Die Werte des vorliegenden Patientenkollektivs befanden sich signifikant (jeweils $p<0,01$, t-Test für Eichstichproben) oberhalb der Normwerte der deutschen Allgemeinbevölkerung (Angermeyer et al. 2000). Verglichen mit der deutschen Allgemeinbevölkerung wiesen die Studiengruppen somit eine höhere subjektive Lebensqualität auf. Zwischen den Studiengruppen A und B gab es keine signifikanten Unterschiede in den vier Domänen „Physisches Wohlbefinden“ $(p=0,34)$, „Psychisches Wohlbefinden“ $(p=0,89)$, „Soziale Beziehungen“ ( $p=0,75)$ und „Umwelt“ ( $p=0,51$, jeweils t-Tests). 


\subsubsection{Hospital Anxiety and Depression Scale - Deutsche Version (HADS-D)}

Die Angst- und Depressionswerte (Angst: 4,08 $\pm 2,39$; Depression: $2 \pm 1,81$ ) liegen in diesem Patientenkollektiv signifikant unterhalb (jeweils $p<0,001$, t-Test für Eichstichproben) der Normwerte einer allgemeinen gesunden Kontrollgruppe (s. Tabelle 13). Ausschließlich ein Patient aus der Gruppe A war auf der Angstskala „auffällig“, wenn man als Auffälligkeitskriterium einen Angstwert $\leq 11$ und/oder Depressionswert $\leq 9$ festlegt (Herrmann-Lingen et al. 2011).

Tabelle 13: Mittelwerte, Standardabweichung, minimale und maximale Werte in beiden Studien und in der Normbevölkerung im HADS-D

\begin{tabular}{|c|c|c|c|c|c|}
\hline & Gruppe & PSI 1-2) & Grupp & $\begin{array}{l}\text { PSI 3-4) } \\
3\end{array}$ & $\begin{array}{c}\text { Normwerte } \\
\text { allgemeine } \\
\text { gesunde } \\
\text { Kontrollpersonen }\end{array}$ \\
\hline \multirow[t]{2}{*}{ Angstwert } & \multicolumn{2}{|c|}{$\begin{array}{c}M W \pm S D \\
3,92 \pm 2,43\end{array}$} & \multicolumn{2}{|c|}{$\begin{array}{c}M W \pm S D \\
4,26 \pm 2,38\end{array}$} & $\mathrm{MW} \pm \mathrm{SD}$ \\
\hline & $\min .0$ & $\max .12$ & $\min .0$ & $\max .9$ & \\
\hline \multirow[t]{2}{*}{ Depressionswert } & \multicolumn{2}{|c|}{$\begin{array}{c}M W \pm S D \\
1,69 \pm 1,81\end{array}$} & \multicolumn{2}{|c|}{$\begin{array}{l}M W \pm S D \\
2,35 \pm 1,8\end{array}$} & $\begin{array}{c}M W \pm S D \\
3,4 \pm 2,6\end{array}$ \\
\hline & $\min .0$ & $\max .7$ & $\min .0$ & $\max .5$ & \\
\hline $\begin{array}{l}\text { HADS-D-Wert } \\
\text { auffällig }\end{array}$ & \multicolumn{2}{|c|}{$\mathrm{n}=1(4 \%)$} & \multicolumn{2}{|c|}{$\mathrm{n}=0(0 \%)$} & $9,9 \%$ \\
\hline
\end{tabular}

Signifikante Unterschiede zwischen den beiden Gruppen bestehen weder auf der Angstskala ( $p=0,63$, t-Test) noch auf der Depressionsskala ( $p=0,21$, t-Test).

\subsection{Bedeutende Einflussvariablen auf die Lebensqualität}

Die nachfolgenden vier Korrelationstabellen geben den Zusammenhang zwischen Gesundheit und Lebensqualität über den Spearmanschen Korrelationskoeffizient $\rho$ (rho) wider. Für Tabelle 14 wurde gemäß den aufgestellten Hypothesen der gerichtete Signifikanzwert für das gesamte Patientenkollektiv errechnet: Patienten ohne parodontale Behandlungsbedürftigkeit sollten eine höhere mundgesundheitsbezogene Lebensqualität (Hypothese I) und eine höhere gesundheitsbezogene Lebensqualität (Hypothese II) aufweisen als Patienten mit parodontaler Behandlungsbedürftigkeit. Die Einflussrichtung von Alter, Angst und Depression auf die gesundheitsbezogene und mundgesundheitsbezogene Lebensqualität war nicht spezifiziert worden (Hypothese III). Somit wird in Tabelle 14 die ungerichtete Signifikanz angegeben. In die Berechnungen 
wurden neben den aggregierten Variablen (Summenskalen) auch theoretisch relevante Einzelvariablen mit aufgenommen. In den Zeilen- bzw. Spaltenköpfen der Tabellen werden in Klammern hinter dem Variablennamen die jeweiligen Erhebungsinstrumente mit aufgeführt. Signifikante Zusammenhänge sind grau unterlegt hervorgehoben. 
Tabelle 14: Spearmansche Korrelation und Signifikanzwerte (einseitig) von Mundgesundheit / Allgemeiner Gesundheit mit gesundheitsbezogener LQ / mundgesundheitsbezogener LQ

\begin{tabular}{|c|c|c|c|c|c|c|c|c|c|}
\hline \multicolumn{2}{|l|}{$\begin{array}{l}\text { Korrelation nach Spearman } \\
\text { (Korrelationskoeffizient } \rho \text { ) }\end{array}$} & \multirow{2}{*}{$\begin{array}{c}\begin{array}{c}\text { MLQ } \\
(\mathrm{OHIP})^{1}\end{array} \\
, 052\end{array}$} & \multirow{3}{*}{\begin{tabular}{|c}
$\begin{array}{c}\text { Körperl. } \\
\text { Gesundheit } \\
\text { (SF-36 } \\
\text { KSK) }\end{array}$ \\
,- 211 \\
, 075
\end{tabular}} & \multirow{3}{*}{$\begin{array}{c}\begin{array}{c}\text { Physische } \\
\text { LQ }\end{array} \\
\text { (WHOQOL) }\end{array}$} & \multirow{3}{*}{\begin{tabular}{|c|}
$\begin{array}{c}\text { Psych. } \\
\text { Gesundheit } \\
\text { (SF-36 } \\
\text { PSK) }\end{array}$ \\
, 088 \\
, 277 \\
\end{tabular}} & \multirow{3}{*}{$\begin{array}{c}\begin{array}{c}\text { Psychische } \\
\text { LQ }\end{array} \\
\text { (WHOQOL) }\end{array}$} & \multirow{3}{*}{$\begin{array}{c}\begin{array}{c}\text { Ruhig \& } \\
\text { Gelassen } \\
\text { (SF-36) }\end{array} \\
-, 284^{*} \\
, 025\end{array}$} & \multirow{3}{*}{ 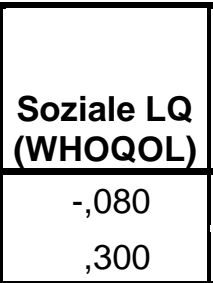 } & \multirow{3}{*}{$\begin{array}{c}\begin{array}{c}\text { Umweltbezogene } \\
\text { LQ (WHOQOL) }\end{array} \\
-, 045 \\
.384\end{array}$} \\
\hline Behandlungsbedarf & $\rho$ & & & & & & & & \\
\hline & Sig. & ,362 & & & & & & & \\
\hline \multirow[t]{2}{*}{ PSI Max (PSI) } & $\rho$ & , 165 &,- 178 &,- 205 & ,027 &,- 160 & \begin{tabular}{|l|}
,- 169 \\
\end{tabular} &,- 119 &,- 086 \\
\hline & Sig. & , 129 & ,113 & ,081 & ,428 & ,142 & ,125 & ,218 & ,285 \\
\hline \multirow[t]{2}{*}{ DMF-T (DMF-T) } & $\rho$ & , 150 &,- 073 &,- 213 & ,092 &,- 024 &,- 099 &,- 144 & ,046 \\
\hline & Sig. & ,152 & ,310 &, 073 & ,267 &, 435 & ,251 &, 173 & ,381 \\
\hline \multirow{2}{*}{$\begin{array}{l}\text { Spezifische Parodontitits- } \\
\text { Symptome (Pcheck) }\end{array}$} & $\rho$ &, $303^{*}$ &,$- 308^{*}$ &,$- 374^{* *}$ & 010 &,- 111 &, 193 &,- 205 & ,091 \\
\hline & Sig. &, 017 &, 017 &, 004 & ,473 & ,230 &, 095 &, 088 & ,273 \\
\hline \multirow{2}{*}{$\begin{array}{l}\text { Empfindliches } \\
\text { Zahnfleisch (Pcheck) }\end{array}$} & $\rho$ & 140 &,- 222 &,$- 275^{*}$ & ,099 &,- 197 & ,059 &,- 237 &,- 015 \\
\hline & Sig. & ,169 & ,065 &, 029 & 251 &, 092 & 345 &, 058 & ,461 \\
\hline \multirow{2}{*}{$\begin{array}{l}\text { Schmerzen im } \\
\text { Mundbereich (OHIP) }\end{array}$} & $\rho$ &, $536^{* *}$ &,- 155 &,- 129 & ,124 & 156 &,- 188 & ,164 & ,161 \\
\hline & Sig. &, 000 & 146 &, 191 & ,201 &, 147 & 101 &, 140 &, 143 \\
\hline \multirow{2}{*}{$\begin{array}{l}\text { Mundgesundheitszustand } \\
\text { (OHIP) }\end{array}$} & $\rho$ &,$- 317^{*}$ & ,024 & ,270* & ,174 & 221 &,- 127 & ,067 & ,077 \\
\hline & Sig. &, 013 & ,435 & ,032 & 119 & ,068 & ,196 & 332 & ,306 \\
\hline \multirow{2}{*}{$\begin{array}{l}\text { Gesundheit (Allgemeiner } \\
\text { Gesundheitsfragebogen) }\end{array}$} & $\rho$ &,- 038 &, $419^{* *}$ & ,382* &,$- 345^{* *}$ & ,154 & ,249* & ,078 & ,268* \\
\hline & Sig. & ,398 &, 002 &, 004 &, 008 & 151 & ,044 & ,304 & ,036 \\
\hline \multirow{2}{*}{$\begin{array}{l}\text { Allg. Gesundheitszustand } \\
\text { (OHIP) }\end{array}$} & $\rho$ &,- 057 & ,251* & $377^{* *}$ & ,252* &, $436^{* *}$ &,- 087 & $294^{*}$ & $327^{*}$ \\
\hline & Sig. & ,348 &, 043 &, 004 &, 042 &, 001 & ,278 & ,025 &, 013 \\
\hline \multirow{2}{*}{$\begin{array}{l}\text { Hoher Blutzucker / } \\
\text { Diabetes (Ihr Allg. } \\
\text { Gesundh.) }\end{array}$} & $\rho$ &,- 142 & $\begin{array}{l}, 202 \\
\end{array}$ &,- 069 & 090 &,- 036 &,$- 249^{*}$ & ,146 & $\begin{array}{l}-057 \\
\end{array}$ \\
\hline & Sig. & ,165 & ,084 & ,321 & 271 & ,405 & ,044 & ,169 & ,354 \\
\hline
\end{tabular}

* Die Korrelation ist auf dem 0,05-Niveau signifikant (einseitig).

${ }^{* *}$ Die Korrelation ist auf dem 0,01-Niveau sehr signifikant (einseitig).

${ }^{1}$ Achtung: Höhere MLQ-Werte stehen für schlechtere Mundgesundheitsbezogene Lebensqualität 
Die signifikanten Korrelationen der Variablen zur Erfassung der Mundgesundheit mit denen zur Lebensqualität ergaben folgende Erkenntnisse (s. Tabelle 14):

- Ein hoher Behandlungsbedarf (operationalisiert über die StudiengruppenZuweisung) geht mit weniger Ruhe und Gelassenheit (operationalisiert über die entsprechende Frage im SF-36; $r=-0,28 ; p=0,03$ ) einher.

- Je höher die Anzahl an Parodontitis-Symptomen (operationalisiert über die PcheckSummenskala), desto schlechter sind mundgesundheitsbezogene Lebensqualität (operationalisiert über die MLQ-Skala im OHIP; $r=0,3 ; p=0,02$ ), körperliche Lebensqualität (operationalisiert über die KSK im SF-36; $r=-0,31 ; p=0,02$ ) sowie physischen Lebensqualität (operationalisiert über die PSK im SF-36; $r=-0,37$; $\mathrm{p}<0,01)$.

- Patienten, die über empfindliches Zahnfleisch berichten (operationalisiert über eine Frage im Pcheck), berichten auch über geringere physische Lebensqualität (operationalisiert über die Physisch-Domäne im WHOQOL; $r=-0,28 ; p=0,03$ ).

- Schmerzen im Mundbereich (operationalisiert über eine Frage im OHIP) stehen in engem Zusammenhang mit geringer mundgesundheitsbezogenen Lebensqualität (operationalisiert über die MLQ-Skala im OHIP; $r=0,54 ; p<0,01$ ).

- Ein als gut eingeschätzter eigener Mundgesundheitszustand (operationalisiert über eine Frage im OHIP) geht statistisch mit einer hohen mundgesundheitsbezogenen Lebensqualität (operationalisiert über die MLQ-Skala im OHIP, $r=-0,32 ; p=0,01$ ) und einer hohen physischen Lebensqualität (operationalisiert über die PhysischDomäne im WHOQOL; $r=0,27 ; p=0,03$ ) einher.

Die signifikanten Korrelationen der Variablen zur Erfassung des allgemeinen

Gesundheitszustands mit denen zur Lebensqualität ergeben Folgendes (s. Tabelle 14):

- Hohe Werte im selbst eingeschätzten Gesundheitszustand (operationalisiert über eine Frage im Fragebogen „Allgemeiner Gesundheitszustand“) gehen einher mit hoher körperlicher Lebensqualität (operationalisiert über die KSK im SF-36; $r=0,42$; $p<0,01$ sowie die Physisch-Domäne im WHOQOL; $r=0,38 ; p<0,01)$, psychischer Lebensqualität (operationalisiert über die PSK im SF-36; $r=-0,35 ; p<0,01$ ) und umweltbezogener Lebensqualität (operationalisiert über die Umwelt-Domäne im WHOQOL; $r=0,27 ; p=0,04$ ) sowie mit Ruhe und Gelassenheit (operationalisiert über eine Frage im SF-36; $r=0,25 ; p=0,04)$. 
- Hohe Werte im selbst eingeschätzten allgemeinen Gesundheitszustand (operationalisiert über eine Frage im OHIP) stehen in Zusammenhang mit hoher körperlicher Lebensqualität (operationalisiert über die KSK im SF-36; $r=0,25 ; p=0,04$ sowie die Physisch-Domäne im WHOQOL; $r=0,38 ; p<0,01)$, psychischer Lebensqualität (operationalisiert über die PSK im SF-36; $r=0,25 ; p=0,04$ sowie die Psychisch-Domäne im WHOQOL; $r=0,44 ; \quad p<0,01)$, sozialer Lebensqualität (operationalisiert über die Sozial-Domäne im WHOQOL; $r=0,29 ; p=0,03$ ) und umweltbezogener Lebensqualität (operationalisiert über die Umwelt-Domäne im WHOQOL; $r=0,33 ; p=0,01$ ).

- Hohe Blutzucker- bzw. Diabetes-Werte (operationalisiert über eine Frage im Fragebogen „Allgemeiner Gesundheitszustand“) standen in negativem Zusammenhang mit Ruhe und Gelassenheit (operationalisiert über eine Frage im SF-36; $r=-0,25 ; p=0,04)$.

Die signifikanten Korrelationen zwischen den erhobenen Emotionswerten (Angst und Depression) mit Lebensqualitätswerten legen die folgenden Zusammenhänge nahe (s. Tabelle 15):

- Je höher die berichteten Angstwerte, desto geringer die physische Lebensqualität (operationalisiert über die Physisch-Domäne im WHOQOL; $r=-0,42 ; p<0,01$ ) und psychische Lebensqualität (operationalisiert über die Physisch-Domäne im WHOQOL; $r=-0,32 ; p=0,03$ ).

- Je höher die berichteten Depressionswerte, desto geringer die Werte in physischer Lebensqualität (operationalisiert über die KSK im SF-36; $r=-0,49 ; p<0,01$ sowie die Physisch-Domäne im WHOQOL; $r=-0,39 ; p<0,01)$, psychischer Lebensqualität (operationalisiert über die Psychisch-Domäne im WHOQOL; $r=-0,4 ; p<0,01$ ) und sozialer Lebensqualität (operationalisiert über die Sozial-Domäne im WHOQOL; r=$0,32 ; p=0,04)$.

- Das Alter stand bei den Befragten in keinem signifikanten Zusammenhang mit ihrer Lebensqualität. 


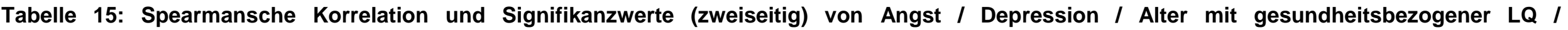
mundgesundheitsbezogener LQ

\begin{tabular}{|c|c|c|c|c|c|c|c|c|c|}
\hline \multicolumn{2}{|c|}{$\begin{array}{l}\text { Korrelation nach } \\
\text { Spearman } \\
\text { (Korrelationskoeffizient } \rho \text { ) }\end{array}$} & MLQ $(\mathrm{OHIP})^{1}$ & $\begin{array}{c}\text { Körperl. } \\
\text { Gesundheit } \\
\text { (SF-36 KSK) } \\
\end{array}$ & $\begin{array}{c}\text { Physische LQ } \\
\text { (WHOQOL) }\end{array}$ & $\begin{array}{c}\text { Psych. } \\
\text { Gesundheit } \\
\text { (SF-36 PSK) }\end{array}$ & $\begin{array}{c}\text { Psychische LQ } \\
\text { (WHOQOL) }\end{array}$ & $\begin{array}{c}\text { Ruhig \& } \\
\text { Gelassen } \\
\text { (SF-36 PSK) } \\
\end{array}$ & $\begin{array}{l}\text { Soziale LQ } \\
\text { (WHOQOL) }\end{array}$ & $\begin{array}{c}\text { Umwelt- } \\
\text { bezogene LQ } \\
\text { (WHOQOL) } \\
\end{array}$ \\
\hline \multirow[t]{2}{*}{ Angst (HADS) } & $\rho$ & ,260 &,- 277 &,$- 416^{* *}$ &,- 273 &,$- 320^{*}$ & ,185 &,- 072 &,- 218 \\
\hline & Sig. & ,072 & ,057 & ,003 & ,060 & ,028 & 207 & ,640 & , 145 \\
\hline \multirow[t]{2}{*}{ Depression (HADS) } & $\rho$ & ,157 &,$- 487^{\star \star}$ &,$- 394^{* *}$ &,- 071 &,$- 401^{* *}$ &,- 103 &,$- 315^{*}$ &,- 257 \\
\hline & Sig. & ,281 &, 000 & ,006 & ,631 & ,005 & ,488 & ,035 & ,084 \\
\hline \multirow[t]{2}{*}{ Alter } & $\rho$ & ,015 &,- 260 &,- 275 & ,213 &,- 071 &,- 158 &,- 067 &,- 051 \\
\hline & Sig. & ,918 & ,074 & ,058 &, 147 & ,634 & ,283 & ,663 & ,736 \\
\hline
\end{tabular}

** Die Korrelation ist auf dem 0,01-Niveau signifikant (zweiseitig).

* Die Korrelation ist auf dem 0,05-Niveau sehr signifikant (zweiseitig). 
Werden die Analysen getrennt nach Geschlecht durchgeführt, kommt es teilweise zu anderen signifikanten Zusammenhängen: einige verschwinden, andere kommen hinzu (s. Tabelle 16 und 17). Zu einer Verschiebung signifikanter Zusammenhänge führt auch eine statistische Kontrolle der Variable "Alter". Allerdings sind die so berechneten Werte aufgrund der geringen Fallzahlen mit äußerster Vorsicht zu interpretieren und werden in dieser Pilotstudie deswegen nicht näher analysiert.

Tabelle 16: Spearmansche Korrelation und Signifikanzwerte (einseitig) von Mundgesundheit / Allgemeiner Gesundheit mit gesundheitsbezogener LQ / mundgesundheitsbezogener LQ (getrennt nach Geschlecht)

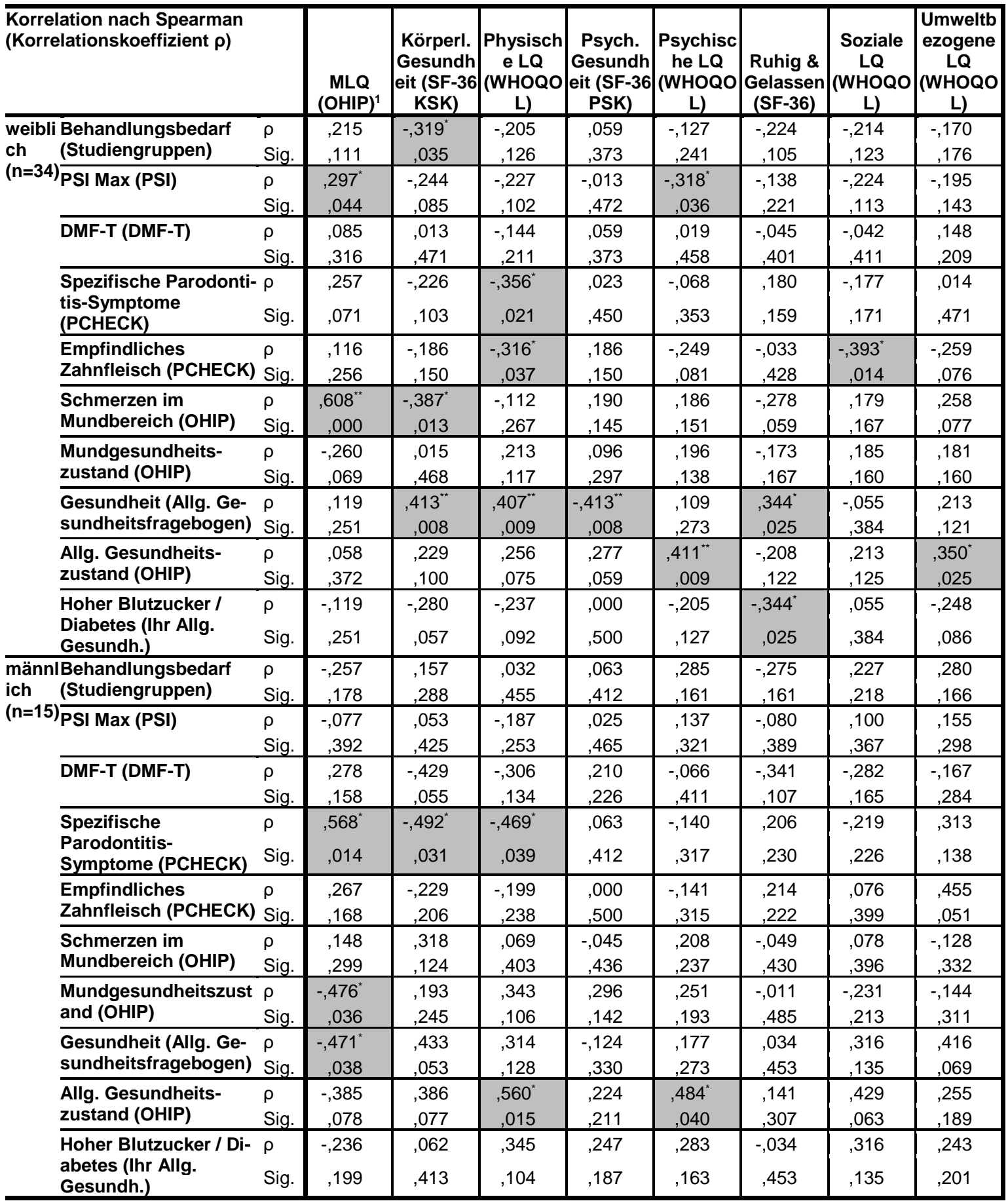

*. Die Korrelation ist auf dem 0,05-Niveau signifikant (einseitig).

**. Die Korrelation ist auf dem 0,01-Niveau signifikant (einseitig)

${ }^{1}$ Achtung: Höhere MLQ-Werte stehen für schlechtere mundgesundheitsbezogene Lebensqualität 
Tabelle 17: Spearmansche Korrelation und Signifikanzwerte (zweiseitig) von Angst / Depression / Alter mit gesundheitsbezogener LQ / mundgesundheitsbezogener LQ (getrennt nach Geschlecht)

\begin{tabular}{|c|c|c|c|c|c|c|c|c|c|c|}
\hline \multicolumn{3}{|c|}{$\begin{array}{l}\text { Korrelation nach Spearman } \\
\text { (Korrelationskoeffizient } \rho \text { ) }\end{array}$} & $\begin{array}{l}\text { MLQ } \\
\text { (OHIP) }\end{array}$ & $\begin{array}{c}\text { Körperl. } \\
\text { Gesundh } \\
\text { eit (SF-36 } \\
\text { KSK) }\end{array}$ & $\begin{array}{c}\text { Physisch } \\
\text { e LQ } \\
\text { (WHOQO } \\
\text { L) }\end{array}$ & $\begin{array}{c}\text { Psych. } \\
\text { Gesundh } \\
\text { eit (SF-36 } \\
\text { PSK) }\end{array}$ & $\begin{array}{c}\text { Psychisc } \\
\text { he LQ } \\
\text { (WHOQO } \\
\text { L) }\end{array}$ & $\begin{array}{c}\text { Ruhig \& } \\
\text { Gelassen } \\
\text { (SF-36) }\end{array}$ & $\begin{array}{c}\text { Soziale } \\
\text { LQ } \\
\text { (WHOQO } \\
\text { L) }\end{array}$ & $\begin{array}{c}\text { Umweltb } \\
\text { ezogene } \\
\text { LQ } \\
\text { (WHOQO } \\
\text { L) }\end{array}$ \\
\hline \multirow{3}{*}{$\begin{array}{l}\text { weibli } \\
\text { ch } \\
(n=34)\end{array}$} & $\begin{array}{l}\text { Angst } \\
\text { (HADS) }\end{array}$ & $\begin{array}{l}\rho \\
\text { Sig. }\end{array}$ & $\begin{array}{l}, 153 \\
, 388\end{array}$ & $\begin{array}{r}-, 287 \\
, 105\end{array}$ & $\begin{array}{r}-, 150 \\
, 403\end{array}$ & \begin{tabular}{r|}
,- 064 \\
, 722
\end{tabular} & $\begin{array}{r}-, 059 \\
, 745\end{array}$ & $\begin{array}{r}-, 052 \\
, 773\end{array}$ & $\begin{array}{l}, 095 \\
, 613\end{array}$ & $\begin{array}{r}-, 143 \\
, 434\end{array}$ \\
\hline & $\begin{array}{l}\begin{array}{l}\text { Depression } \\
\text { (HADS) }\end{array} \\
\end{array}$ & $\begin{array}{l}\rho \\
\text { Sig. }\end{array}$ & $\begin{array}{l}158 \\
, 372 \\
\end{array}$ & $\begin{array}{r}-, 309 \\
, 080 \\
\end{array}$ & $\begin{array}{r}-, 306 \\
, 084 \\
\end{array}$ & $\begin{array}{r}-, 001 \\
, 996 \\
\end{array}$ & $\begin{array}{r}-, 123 \\
, 495 \\
\end{array}$ & $\begin{array}{r}-, 136 \\
, 450 \\
\end{array}$ & $\begin{array}{r}-, 120 \\
, 521 \\
\end{array}$ & $\begin{array}{r}-, 188 \\
, 303 \\
\end{array}$ \\
\hline & Alter & $\begin{array}{l}\rho \\
\rho \\
\text { Sig. }\end{array}$ & $\begin{array}{r}-, 065 \\
, 715\end{array}$ & $\begin{array}{r}-, 325 \\
, 065\end{array}$ & $\begin{array}{r}-, 111 \\
, 540\end{array}$ & $\begin{array}{l}, 451^{* *} \\
, 008\end{array}$ & $\begin{array}{l}, 059 \\
, 742\end{array}$ & $\begin{array}{r}-, 462^{* *} \\
, 007 \\
\end{array}$ & $\begin{array}{l}, 001 \\
, 994\end{array}$ & $\begin{array}{l}, 111 \\
, 546\end{array}$ \\
\hline \multirow{3}{*}{$\begin{array}{l}\text { männl } \\
\text { ich } \\
(n=15)\end{array}$} & $\begin{array}{l}\text { Angst } \\
\text { (HADS) }\end{array}$ & $\begin{array}{l}\rho \\
\rho \\
\text { Sig. }\end{array}$ & $\begin{array}{l}, 490 \\
, 064\end{array}$ & $\begin{array}{r}-, 559^{*} \\
, 030\end{array}$ & $\begin{array}{r}-, 788^{* *} \\
, 000\end{array}$ & $\begin{array}{r}-, 543^{*} \\
, 037\end{array}$ & $\begin{array}{r}-, 757^{* *} \\
, 002\end{array}$ & $\begin{array}{l}282 \\
, 308\end{array}$ & $\begin{array}{r}-, 303 \\
292\end{array}$ & $\begin{array}{r}-, 366 \\
, 198\end{array}$ \\
\hline & $\begin{array}{l}\begin{array}{l}\text { Depression } \\
\text { (HADS) }\end{array} \\
\end{array}$ & $\begin{array}{l}\rho \\
\rho\end{array}$ & $\begin{array}{l}, 445 \\
, 096 \\
\end{array}$ & $\begin{aligned}-, 727^{* *} \\
, 002 \\
\end{aligned}$ & $\begin{array}{r}-, 586^{*} \\
, 022 \\
\end{array}$ & $\begin{array}{r}-, 295 \\
, 286 \\
\end{array}$ & $\begin{aligned}, 733^{* *} \\
, 003 \\
\end{aligned}$ & $\begin{array}{ll}, 023 \\
, 936 \\
\end{array}$ & $\begin{array}{r}-, 418 \\
, 136 \\
\end{array}$ & $\begin{aligned}-, 261 \\
, 368 \\
\end{aligned}$ \\
\hline & Alter & $\begin{array}{l}\rho \\
\text { Sig. }\end{array}$ & $\begin{array}{l}, 213 \\
, 445\end{array}$ & $\begin{array}{r}-, 266 \\
-338\end{array}$ & $\begin{array}{r}-, 487 \\
, 066\end{array}$ & $\begin{array}{r}-, 158 \\
, 573\end{array}$ & $\begin{array}{r}-, 308 \\
, 284\end{array}$ & $\begin{array}{l}\text {,300 } \\
\text { 2777 }\end{array}$ & $\begin{array}{r}-, 129 \\
, 660 \\
\end{array}$ & $\begin{array}{r}-, 356 \\
, 212 \\
\end{array}$ \\
\hline
\end{tabular}

**. Die Korrelation ist auf dem 0,01-Niveau signifikant (zweiseitig).

*. Die Korrelation ist auf dem 0,05-Niveau signifikant (zweiseitig).

\subsection{Zusammenfassung der wichtigsten Ergebnisse}

Von den an der Studie teilnehmenden 49 Patienten waren knapp 70\% Frauen. Das Durchschnittsalter aller Patienten lag bei $49 \pm 9,7$ Jahren. Die deskriptiven und inferenzstatistischen Analysen der erhobenen Daten werden im Folgenden zusammengefasst.

In einem ersten Analyseschritt wurden die Gesundheits- und Lebensqualitätswerte der zwei Studiengruppen „ohne parodontale Behandlungsbedürftigkeit“ (Gruppe A) und „mit parodontaler Behandlungsbedürftigkeit“ (Gruppe B) miteinander verglichen:

- 26 Patienten waren ohne (Gruppe A: PSI 1-2), 23 mit parodontaler Behandlungsbedürftigkeit (Gruppe B: PSI 3-4). Die beiden Gruppen unterschieden sich signifikant nach ihrem Alter (Gruppe $A$ jünger; $p=0,03$ ); jedoch nicht bezüglich Familienstand, Ausbildungsgrad und Rauchgewohnheiten.

- Der mittlere DMF-T-Wert in Gruppe A lag bei 14,9 \pm 4,4 und in Gruppe B bei 17,7 $\pm 3,9$ $(p=0,02)$.

- Hinsichtlich der Parodontitis-Symptome (nach Parodontitis-Checkliste) zeigten sich keine signifikanten Unterschiede zwischen den Studiengruppen.

- Die beiden Untersuchungsgruppen unterschieden sich nicht signifikant bezüglich ihrer Einschätzung des eigenen Mundgesundheitszustands (erhoben mittels OHIP-G14), wohl aber hinsichtlich ihres allgemeinen Gesundheitszustands $(p=0,05)$. Der OHIP MLQ-Gesamtwert differiert nicht signifikant zwischen den beiden Gruppen, jedoch zeigte sich ein signifikanter Unterschied hinsichtlich der Schmerzen im Mundbereich $(p<0,01)$. 
- Aus Gruppe A gaben im Allgemeinen Gesundheitsfragebogen 92\% der Studienteilnehmer an, "gesund“ zu sein; in Gruppe B waren es 96\%. Patienten aus Gruppe B berichten signifikant häufiger über Schlafprobleme $(p=0,04)$ sowie über hohen Blutzucker / Diabetes $(p=0,01)$.

- Bei den erhobenen Daten zu subjektivem Gesundheitszustand und krankheitsbedingter Belastungen (Lebensqualität) über den SF-36 waren weder auf der körperlichen Summenskala (KSK) noch auf der psychischen Summenskala (PSK) Unterschiede zwischen den Studiengruppen feststellbar.

- Die allgemeine subjektive Lebensqualität (gemessen über den WHOQOL-BREF) zeigte in allen vier Domänen „Physisches Wohlbefinden“, „Psychisches Wohlbefinden“, „Soziale Beziehungen“ und „Umwelt“ keine signifikanten Unterschiede.

- Die mittels HADS-D erhobenen Angst- und Depressionswerte ergaben keine signifikanten Unterschiede zwischen den beiden Gruppen.

In einem zweiten Analyseschritt wurde dann die korrelative Beziehung von Gesundheit und Lebensqualität ermittelt. Zum Zusammenhang von Mundgesundheit und Lebensqualität lassen sich die Ergebnisse wie folgt zusammenfassen:

- Ein hoher Behandlungsbedarf ging mit weniger Ruhe und Gelassenheit einher ( $r=-$ $0,28 ; p=0,03)$.

- Je höher die Anzahl an Parodontitis-Symptomen, desto schlechter waren mundgesundheitsbezogene, körperliche und physische Lebensqualität $(r \geq 0,3$; $p \leq 0,02)$.

- Patienten, die über empfindliches Zahnfleisch berichteten, berichteten auch über geringere physische Lebensqualität $(r=-0,28 ; p=0,03)$.

- Schmerzen im Mundbereich standen in engem Zusammenhang mit geringer mundgesundheitsbezogenen Lebensqualität $(r=0,54 ; p<0,01)$.

- Ein als gut eingeschätzter eigener Mundgesundheitszustand ging statistisch mit einer hohen mundgesundheitsbezogenen Lebensqualität und einer hohen physischen Lebensqualität einher, $r \geq 0,27 ; p \leq 0,03$ ).

Zum Zusammenhang von allgemeinem Gesundheitszustand und Lebensqualität erbringt die vorliegende Arbeit die folgenden Ergebnisse:

- Hohe Werte im selbst eingeschätzten Gesundheitszustand gingen einher mit hoher körperlicher, psychischer und umweltbezogener Lebensqualität $(r \geq 0,27 ; p \leq 0,04)$.

- Hohe Werte im allgemeinen Gesundheitszustand standen in Zusammenhang mit hoher körperlicher, psychischer, sozialer und umweltbezogener Lebensqualität $(r \geq 0,25 ; p \leq 0,04)$. 
- Hohe Blutzucker- bzw. Diabetes-Werte standen in negativem Zusammenhang mit Ruhe und Gelassenheit $(r=-0,25 ; p=0,04)$.

Schließlich wurden auch die Einflussvariablen Angst, Depression und Alter auf die Lebensqualität näher untersucht:

- Je höher die berichteten Angstwerte, desto geringer die hhysische und psychische Lebensqualität $(r \geq 0,32 ; p \leq 0,03)$.

- Je höher die berichteten Depressionswerte, desto geringer die Werte in physischer, psychischer und sozialer Gesundheit ( $r \geq 0,32 ; p \leq 0,04)$.

- Das Alter stand bei den Befragten in keinem signifikanten Zusammenhang mit ihrer Lebensqualität.

Abschießend ist noch anzumerken, dass die Variablen Geschlecht und Alter zwei bedeutsame Kovariaten darstellten. Aufgrund der geringen Fallzahlen wurde auf eine detaillierte Auswertung jedoch verzichtet. 


\section{Diskussion}

Die Parodontitis ist mit einer Prävalenz von über $50 \%$ in der erwachsenen Bevölkerung als „Volkskrankheit“ zu bezeichnen (Jordan 2016). Um die Auswirkungen parodontaler Krankheitszustände auf die mundgesundheitsbezogene Lebensqualität besser zu erfassen, stehen mittlerweile etablierte Messinstrumente zur Verfügung, z. B. der OHIP-G14 (John und Micheelis 2003). Nicht nur die klinische Feststellung von „Krankheit“, sondern eine ganzheitliche Betrachtung des Menschen inklusive seiner Lebensqualität ist empfehlenswert und im Fokus aktueller Forschung. Bislang gibt es nur wenige klinische Studien, die sich mit dem Einfluss der parodontalen Verhältnisse auf die allgemeine und/oder mundgesundheitsbezogene Lebensqualität befasst haben.

Diese Untersuchung befasste sich mit der mundgesundheitsbezogenen Lebensqualität unter Berücksichtigung der allgemeinen Lebensqualität und der gesundheitsbezogenen Lebensqualität sowie weiterer Einflussfaktoren bei Patienten mit und ohne parodontale Behandlungsbedürftigkeit. In beiden Studiengruppen berichteten die Patienten von Parodontitis-spezifischen Symptomen. Die am häufigsten genannten Symptome in beiden Gruppen waren empfindliches Zahnfleisch, Zahnfleischbluten, zurückgehendes Zahnfleisch und Zahnüberempfindlichkeit. Diese Symptome können bei einer Parodontitis, jedoch auch bereits bei einer Gingivitis auftreten. Da in der Gesamtbevölkerung nur 7\% der Erwachsenen ein völlig gingivitisfreies Gebiss vorzeigen können (Micheelis und Schiffner 2006), ist die subjektive Wahrnehmung dieser Symptome auch in der Gruppe A nachvollziehbar. Kein einziger Patient dieses Patientenkollektivs konnte einen PSI von null und damit völlige Entzündungsfreiheit vorweisen. Allein auf eine Parodontitis beschränkte Symptome, die vom Patienten wahrnehmbar sind, sind die Veränderung der Zahnstellung, starke Zahnlockerungen, Zahnverlust und persistierender Mundgeruch. Wobei Mundgeruch von Patienten subjektiv sehr unterschiedlich bewertet wird und nicht als objektives Symptom für eine Parodontitis angesehen werden kann ( $\mathrm{Ng}$ und Leung 2006). Bei Betrachtung der Mittelwerte der Parodontitis-Checkliste war eine leichte Tendenz, wenn auch nicht signifikant, zu einer höheren Anzahl Parodontitis-spezifischer Symptome in der behandlungsbedürttigen Gruppe B zu erkennen (A: 1,92 $\pm 1,72$; B: 2,7 $\pm 2,16$ ). Diese Tendenz spiegelte sich ebenfalls bei folgenden Symptomen wider: schmerzendes und empfindliches Zahnfleisch, Zahnüberempfindlichkeit, Zahnlockerung und Zahnwanderung. Diese wurden in Gruppe B etwas häufiger genannt und bezüglich „empfindliches Zahnfleisch“ existiert sogar ein signifikanter Zusammenhang. Bei $\mathrm{Ng}$ und Leung (2006) waren die meistgenannten Symptome schmerzende und zurückgehende Gingiva und wurden von einem Sechstel der Patienten berichtet. Patienten aus der Gruppe A kamen 
häufiger zu beschwerdebedingten Kontrolluntersuchungen (A: 31\%; B: 17\%) und ließen häufiger regelmäßig eine professionelle Zahnreinigung durchführen (A: 81\%; B: 65\%). Erwartet wurde eine umgekehrte Verteilung. Behandlungsbedürftige Patienten hätten häufiger untersuchungswürdige Beschwerden haben sollen. Eine mögliche, jedoch spekulative Erklärung ist ein höheres Bewusstsein für Mundgesundheit in der Gruppe A und damit der Wunsch, ggf. bereits leichte Beschwerden professionell abklären und behandeln zu lassen. Ein Indiz dafür mag auch der signifikant niedrigere DMF-T-Wert in Gruppe A sein (A: 14,9 $\pm 4,4$; B: 17,7 $\pm 3,9$ ).

Bei der Bewertung der mundgesundheitsbezogenen Lebensqualität mit Hilfe des Oral Health Impact Profile (OHIP) gab die Mehrzahl der Patienten an, dass ihr allgemeiner Gesundheitszustand und Mundgesundheitszustand gut bis ausgezeichnet war. In der Gruppe A wurde tendenziell der allgemeine Gesundheitszustand $(2,62 \pm 0,8)$ von den Patienten im Mittel etwas besser eingeschätzt als ihr Mundgesundheitszustand $(2,23 \pm 0,77)$. Auch hierfür könnte vermutlich eine mögliche Erklärung in einem höheren Bewusstsein und Anspruch an die Mundgesundheit dieser Patientengruppe liegen. So schätzen Patienten aus der Gruppe A gegenüber Patienten der Gruppe B ihren allgemeinen Gesundheitszustand auch signifikant als besser ein. Patienten aus Gruppe B berichten signifikant häufiger über Schlafprobleme sowie über hohen Blutzucker / Diabetes.

Signifikante Unterschiede in der mundgesundheitsbezogenen Lebensqualität (MLQ) zwischen den beiden Gruppen gab es nicht, jedoch eine leicht erkennbare Tendenz, dass die behandlungsbedürftige Gruppe $B$ etwas höhere Werte und damit eine schlechtere MLQ aufwies (A: $2,12 \pm 2,58$; B: $2,48 \pm 3,1$ ). Vor allem in der Subskala „Schmerzen“ hatte Gruppe B eine erhöhte Prävalenz. Für das Item „Schmerzen im Mundbereich“ (das in Gruppe B auch signifikant häufiger berichtet wurde) lag die Prävalenz bei 39\%. Für das Item „Gefühl der Unsicherheit“ lag die Prävalenz in Gruppe B bei $26 \%$ und für das Item „Unangenehm, bestimmte Nahrungsmittel zu essen“ lag die Prävalenz bei 22\%. In der DMS IV-Studie (2006) waren die Items „Schmerzen im Mundbereich“ und „Unangenehm, bestimmte Nahrungsmittel zu essen“ ebenfalls die mit der größten Prävalenz (25-35\%), jedoch in der allgemeinen Erwachsenen- wie auch Senioren-Gruppe unabhängig von einer Parodontitis und nicht - wie in der vorliegenden Studie - in der Gruppe mit der parodontalen Behandlungsbedürftigkeit. Die Prävalenz in der Allgemeinbevölkerung ist also ähnlich hoch wie in der behandlungsbedürftigen Gruppe B, hier zeigt sich der mögliche Einfluss anderer Faktoren wie herausnehmbarer Zahnersatz und hohe Anzahl fehlender Zähne in der DMS IV-Studie (2006).

Andere Untersuchungen zeigten deutliche Auswirkungen einer Parodontitis auf die MLQ. $\mathrm{Ng}$ und Leung (2006) fanden eine signifikante Assoziation zwischen einer höheren Anzahl parodontitisspezifischer Symptome, wie geschwollener, schmerzender und 
zurückgehender Gingiva, Zahnlockerungen, Mundgeruch und Zahnschmerzen, in Relation zu höheren OHIP-Werten. Zu beachten ist jedoch, dass bei Ng und Leung (2006) Patienten mit wenig Zugang zur zahnärztlichen Versorgung in die Untersuchung eingeschlossen wurden. Diese Patienten hatten kariöse und zerstörte Zähne, fehlende Frontzähne und fehlende Stützzonen, insgesamt also insuffizient versorgte Gebisse mit teilweise nur noch fünf vorhandenen Zähnen ( $\mathrm{Ng}$ und Leung 2006). Das Studiendesign der vorliegenden Studie hatte sehr strenge Einschluss- und Ausschlusskriterien, um mögliche Störfaktoren zu minimieren. Am entscheidendsten war der Ausschluss von Patienten mit herausnehmbarem Zahnersatz, mehr als 8 fehlenden Zähnen und kariösen, insuffizient versorgten Zähnen. Herausnehmbarer Zahnersatz ersetzt die Funktion der Zähne oft unzureichend (John 2005). Dies belegen John und Micheelis (2003), bei denen über die Hälfte der Probanden über Probleme mit dem herausnehmbaren Zahnersatz klagte.

Verschiedene Untersuchungen zeigten eine Verschlechterung der MLQ mit einer erhöhten Anzahl fehlender Zähne (Gerritsen et al. 2010; Micheelis und Schiffner 2006; Ohrn und Jönsson 2012). Steele et al. (2004) stellten fest, dass Personen mit unter 17 Zähnen (Großbritannien) bzw. unter 21 Zähnen (Australien) signifikant schlechtere OHIP-Werte aufweisen. Die besten OHIP-Werte erhalten Personen mit über 25 Zähnen (Steele et al. 2004). Diese Werte werden von Cunha-Cruz et al. (2007) ebenfalls bestätigt.

Inwieweit kariöse Zähne die MLQ mindern ist noch unklar. Eine signifikante Assoziation zwischen kariösen Zähnen und einer eingeschränkten MLQ haben Ng und Leung (2006) nicht gefunden. Die deutsche Mundgesundheitsstudie DMS IV (2006) bescheinigt ebenfalls keine Abhängigkeit des OHIP-G14-Summenwertes vom Schweregrad des Kariesbefalls. Hohe Kariesschweregrade schienen aber mit der Einschränkung der MLQ statistisch verbunden zu sein (Micheelis und Schiffner 2006). Fotedar et al. (2014) fanden dagegen signifikante Assoziationen zwischen kariös zerstörten Zähnen, Parodontitis und fehlenden Zähnen. Daneben erkannten verschiedene Arbeitsgruppen, dass eine stärkere Ausbreitung und ein höherer Schweregrad der Parodontitis die MLQ zunehmend verschlechtert (Bernabé und Marcenes 2010; Ng und Leung 2006; Al Habashneh et al. 2012; Cunha-Cruz et al. 2007; Needleman et al. 2004). Starke Einschränkungen der MLQ fanden sich bei Patienten, die jeweils zu einem speziellen Patientenklientel von Parodontitispatienten gehörten und in spezialisierte Parodontitispraxen gekommen waren, um sich behandeln zu lassen. Diese Patienten hatten Kenntnis davon, dass eine parodontale Erkrankung vorliegt und somit vielleicht auf Grund der eingeschränkten MLQ, den Wunsch nach einer Behandlung (Cunha-Cruz et al. 2007; Needleman et al. 2004; Saito et al. 2010). Insgesamt muss wahrscheinlich die Probandenauswahl beim Vergleich von Studien genau berücksichtigt werden. In Industrieländern haben Patienten einen guten Zugang zur modernen zahnmedizinischen Versorgung, in Entwicklungs- und Schwellenländern ist die 
Versorgung, wenn überhaupt zugängig, oft auf die Schmerztherapie beschränkt. Auf Grund fehlender Zähne und sich daraus ergebener Stützzonenverluste oder fehlender und nicht ersetzter Zähne im Frontbereich können funktionelle, phonetische und ästhetische Beeinträchtigungen auftreten. Kariöse, zerstörte, insuffizient versorgte Zähne können zu Schmerzen, scharfen Kanten, Mundgeruch, funktionellen Problemen, wie auch phonetischen und ästhetischen Beeinträchtigungen führen. Daraus ergeben sich viele mögliche Faktoren, die neben der eigentlichen parodontalen Erkrankung, die mundgesundheitsbezogene Lebensqualität beeinflussen könnten (Müller und von Steinbüchel 2005; John 2004; Steele et al. 2004; Ng und Leung 2006).

Bei den Fragebögen „Ihr allgemeiner Gesundheitszustand“ (Von Steinbüchel et al. 2006), „SF-36“ (Bullinger und Kirchberger 1998), „WHOQOL-BREF“ (Angermeyer et al. 2000) und „HADS-S“ (Herrmann-Lingen et al. 2011) waren auf den Summenskalen keine signifikanten Unterschiede zwischen den beiden Studiengruppen A und B feststellbar. Auf Ebene der Einzelfragen unterscheiden sich die Gruppen hingegen. Ein höherer Behandlungsbedarf (Gruppe B) geht mit weniger Ruhe und Gelassenheit einher. Je höher die Anzahl an Parodontitis-Symptomen, desto schlechter sind mundgesundheitsbezogene, körperliche und physische Lebensqualität. Patienten, die über empfindliches Zahnfleisch berichten, berichten auch über geringere physische Lebensqualität. Schmerzen im Mundbereich stehen in engem Zusammenhang mit geringer mundgesundheitsbezogenen Lebensqualität. Ein als gut eingeschätzter eigener Mundgesundheitszustand geht statistisch mit einer hohen mundgesundheitsbezogenen Lebensqualität und einer hohen physischen Lebensqualität einher.

In diesem Patientenkollektiv gab es weder Schwerkranke noch immobile Patienten, dieses kann auf die Ausschlusskriterien zurückgeführt werden und ist womöglich der Grund, dass dieses Patientenkollektiv beim SF-36, WHOQOL-BREF und HADS-S bessere Werte als die Normbevölkerung aufwies. Somit ließen sich beeinflussende Kofaktoren auf die MLQ reduzieren. Für spätere Untersuchungen sollten jedoch vor allem Geschlecht und Alter statistisch kontrolliert werden.

Diese Untersuchung testete als Machbarkeitsstudie verschiedene Studienparameter (Fragebögen) und wies dementsprechend nur eine geringe Probandenanzahl auf. Durch die geringe Probandenanzahl ist die Aussagekraft dieser Ergebnisse prinzipiell eingeschränkt. Besonders hervorzuheben ist, dass im Rahmen dieser Studie sehr viele Parameter untersucht worden sind und dies in so einem komplexen Umfang erstmalig in der Praxis durchgeführt worden ist. Es wurden nur validierte wissenschaftlich gut erforschte Fragebögen verwendet. Die klinischen Parameter waren die Erhebung des PSI und ein ausführlicher zahnärztlicher Befund. Die Einteilung in zwei Gruppen, eine mit und eine ohne parodontale Behandlungsbedürftigkeit, erfolgte anhand des PSI (Gruppe A: PSI 1-2; 
Gruppe B: PSI 3-4). Weitere Parameter waren parodontitisspezifische Symptome, allgemeiner und subjektiver Gesundheitszustand, allgemeine subjektive Lebensqualität, mundgesundheitsbezogene Lebensqualität, die Erfassung von Angst und Depressivität, wie auch soziodemografische Daten.

Das Hauptziel dieser Untersuchung war es herauszufinden, ob diese Studie unter Praxisbedingungen als großangelegte Studie mit diesen Studienparametern umsetzbar ist. Es hat sich herausgestellt, dass diese Untersuchung sehr aufwendig, aber in einer abgewandelten Form durchaus empfehlenswert ist. In so einer umfassenden ganzheitlichen Form gibt es derzeit noch keine Studien. Die strengen Ein- und Ausschlusskriterien sind sinnvoll und sollten beibehalten werden. Vor allem können durch den Ausschluss von Patienten mit herausnehmbaren Zahnersatz, mehr als acht fehlenden Zähnen und kariösen, insuffizient versorgten Zähnen, mitbeeinflussende Faktoren auf die MLQ minimiert werden. Hier sind besonders Patienten mit einer fortgeschrittenen Parodontitis, aber noch nicht zu vielen verlorenen Zähnen, von besonderem Interesse.

Die ausführliche dentale Befundung gehört zur Routineuntersuchung und stellt keinen Mehraufwand der Studie dar. Die Parodontitis-Checkliste ( $\mathrm{Ng}$ und Leung 2006) ist ein sinnvolles Instrument zur Feststellung subjektiver Parodontitis-Symptome und sollte beibehalten werden. Die Eignung des PSI als Screening-Index, ebenfalls zur Routineuntersuchung gehörend, sollte für die Aufteilung in eine behandlungsbedürftige Gruppe und gesunde Kontrollgruppe nochmals genauer analysiert werden. Bei der behandlungsbedürftigen Gruppe sollte zusätzlich ein vollständiger Parodontalstatus erhoben werden, um den Schweregrad genauer erfassen zu können. Bei der Gruppeneinteilung sind zudem Kontrollvariablen wie Alter und Geschlecht zu berücksichtigen.

Der OHIP-G14 (John et al. 2004) ist ein geeignetes Instrument zur Erfassung der MLQ. Parodontitis-spezifischere Fragen aus der Langversion des OHIP-G53 wie „empfindliche Zähne“, „Zahnschmerzen“ und „Zahnfleischschmerzen“ werden bereits mit der Parodontitis-Checkliste abgefragt, so dass die Langversion keinen Mehrwert brächte. Alternativ könnte der Geriatric Oral Health Assessment Index (GOHAl) verwendet werden. Ohrn und Jönsson (2012) haben beide Instrumente verglichen und keine signifikanten Unterschiede festgestellt. Jedoch gaben die Autoren an, dass sich die größere Vielfalt der Fragen zu funktionellen Aspekten (Kauen, Schlucken, Abbeißen) beim GOHAl evtl. als besser für Parodontitis-Patienten erweisen könnte (Ohrn und Jönsson 2012). Auf Grund fehlender Erfahrung kann hier jedoch keine Empfehlung erfolgen.

Der Fragebogen zum allgemeinen Gesundheitszustand (Von Steinbüchel et al. 2006) enthält neben anamnestischen Fragen zu Erkrankungen, spezifische Fragen aus psychischen und funktionellen Bereichen wie z. B. „Geruchs- oder Geschmacksprobleme“, 
„Schlafprobleme“, „Mangel an Energie“ und „Bewegungsschwierigkeiten“. Im OHIP-G14 wird nach einer Beeinträchtigung des Geschmacksinns gefragt (Frage 4) und die spezifischen Bereiche werden ebenfalls im WHOQOL-BREF abgefragt (Fragen 10, 16, 15). Somit könnte der Fragebogen zum allgemeinem Gesundheitszustand (Von Steinbüchel et al. 2006) durch einen kürzeren aber validierten Gesundheitsfragebogen ersetzt werden. Die Verwendung von zwei Fragebögen zur subjektiven Erfassung der allgemeinen Lebensqualität SF-36 (Bullinger und Kirchberger 1998) und WHOQOL-BREF (Angermeyer et al. 2000) ist sehr zeitintensiv. Auch wenn diese teilweise unterschiedliche Schwerpunkte haben, ist die Verwendung beider letztendlich nicht von ausreichendem Mehrwert. Das Ausfüllen eines dieser Fragebögen nimmt jeweils ca. zehn Minuten in Anspruch. Zu empfehlen ist die Reduktion auf einen dieser Fragebögen. Es erscheint sinnvoll für die ganzheitliche Betrachtung zu sein, neben der mundgesundheitsbezogenen Lebensqualität auch die allgemeine Lebensqualität zu erfassen. Die Verwendung des WHOQOL-BREF bietet sich mehr an, da neben physischen und psychischen Wohlbefinden auch soziale Aspekte und Umweltbedingungen gut ausgearbeitet werden können. Der SF-36 konzentriert sich stärker auf die körperliche und psychische Funktionsfähigkeit. Alternativ könnte bei Bedarf die Kurzversion SF-12 verwendet werden. Der HADS-D (HerrmannLingen et al. 2011) wurde gezielt für den Einsatz bei Personen in somatisch-medizinischen Einrichtungen entwickelt, um Angst und Depressivität zu erfassen. Die Erfassung von diesen psychischen Zuständen ist prinzipiell sinnvoll, denn es ist anzunehmen, dass jemand der depressiv ist und insgesamt mit seinem Leben unzufrieden, seine MLQ auch ohne objektive Gründe schlechter bewerten wird. Inwieweit jedoch ein Extrafragebogen sinnvoll ist, bleibt fraglich. Der WHOQOL-BREF beinhaltet Fragen, die eine vorliegende Depression vermuten lassen, wie z. B. „Betrachten Sie Ihr Leben als sinnvoll (6)“, „wie zufrieden sind Sie mit sich selbst (19)“ und „wie häufig haben Sie negative Gefühle wie Traurigkeit, Verzweiflung, Angst oder Depression (26)“. Natürlich ist die Erfassung einer Depression oder von Angstzuständen mit dem HADS-D präziser, jedoch ist der Mehrnutzen für diese Studie fraglich. Ein soziodemografischer Fragebogen ist erforderlich und sollte die gängigen Fragen für statistische Erhebungen beinhalten und zwar nach Geschlecht, Alter, Familienstand, Ausbildungsgrad und Berufstätigkeit. Der hier verwendete soziodemografische Fragebogen (Von Steinbüchel et al. 2006) enthält zusätzlich Fragen zu sozialen, emotionalen und körperlichen Aspekten. Teilweise werden diese Bereiche ebenfalls durch den WHOQOL-BREF abgedeckt, so dass hier ein kürzerer, jedoch ebenfalls validierter, soziodemografischer Fragebogen verwendet werden könnte. Die Frage nach dem Tabakkonsum ist wichtig und sollte ebenfalls im Fragebogen enthalten sein. 
Insgesamt sollte diese Studie durch Reduktion ganzer Fragebögen und der Verwendung kürzerer Fragebögen zur Soziodemografie und zum Gesundheitszustand weniger zeitintensiv und somit besser im Praxisalltag durchührbar werden. Das momentane Studiendesign ist für das Praxisteam und den Patienten zu umfangreich und zeitintensiv und deshalb in dieser Form kaum unter Praxisbedingungen umsetzbar. Die teilweise sehr ähnlichen Fragen, die zwar feine Unterschiede beinhalten, werden vom Patienten oft als gleich empfunden. Die maximal zumutbare Interviewdauer ist allgemein für Patienten, neben der tatsächlichen Zeitdauer, von verschiedenen Faktoren wie dem Thema, den Zielpersonen wie auch dem Sinn und Zweck abhängig (Micheel 2010). Die Patienten haben in der vorliegenden Untersuchung rund 160 Fragen beantwortet und waren eigentlich nur zur Kontrolluntersuchung beim Zahnarzt gekommen. Der Zahnarztbesuch an sich ist für viele Patienten kein positives Ereignis, so dass eine zu starke Verlängerung des Aufenthaltes in der Praxis die Ausfüllbereitschaft des Patienten herabsenken kann. Es wäre möglich die allgemeinen Fragebögen den Patienten mit nach Hause zu geben. Dieses könnte jedoch mit einem höheren Mehraufwand für das Praxisteam durch ggf. nötige telefonische Erinnerungen an die Fragebögen verbunden sein. Zudem wäre eine erhöhte Non-Responderrate und Abbruchrate zu erwarten. Durch den Verzicht auf die Fragebögen SF-36 und HADS-D würden bereits 50 Fragen wegfallen. Zusätzlich könnten durch die Verwendung kürzerer Fragebögen zur Soziodemografie und zum Gesundheitszustand von den momentanen 48 Fragen ebenfalls einige dezimiert werden. Der Detaillierungsgrad der Studie muss hier gegen die Ausfüllbereitschaft des Patienten und die Umsetzbarkeit für das Praxisteam abgewogen werden. Einen rentablen Mehraufwand würde die Aufnahme des Parodontalstatus in der behandlungsbedürftigen Gruppe B (PSI 3-4) bedeuten. Dadurch könnten bessere Aussagen über den Einfluss des Schweregrades der parodontalen Erkrankung auf die MLQ getroffen werden.

Zusammenfassend ist die Verwendung folgender Fragebögen empfehlenswert:

- Parodontitis-Checkliste

- Oral Heath Impact Profile (OHIP-G14)

- World Health Organization Quality of Life Scale (WHOQOL-BREF)

- Validierte Fragebögen zur Soziodemografie und zum Gesundheitszustand

Trotz der Reduzierung der Fragen bleibt diese Studie recht aufwendig und die durchführenden Praxen müssen sich dessen bewusst sein. Durch eine gute Zusammenarbeit im Team ist der Aufwand zu bewältigen. Manche Studienparameter können vom Arzt auf geschultes Fachpersonal delegiert werden, wie z. B. die Aufnahme des Parodontalstatus durch Dentalhygieniker/innen. Außerdem ist eine Auswahl von 
Patienten, die bereits ein gutes Vertrauensverhältnis zu der jeweiligen Praxis haben, zu empfehlen, da teilweise intime Frage gestellt werden (WHOQOL-BREF Frage 21. "wie zufrieden sind Sie mit Ihrem Sexualleben“). Also sollten die Praxisinhaber überlegen, ob sie Neupatienten, die zum ersten Mal in die Praxis kommen, in die Studie einbeziehen möchten.

Die in dieser Pilotstudie erhobenen Daten erlauben keine eindeutige Beantwortung der drei aufgestellten Hypothesen. Wird die parodontale Behandlungsbedürftigkeit über den PSIWert operationalisiert, so ließ sich die Hypothese I (Patienten ohne eine parodontale Behandlungsbedürftigkeit haben eine geringere Beeinträchtigung der mundgesundheitsbezogenen Lebensqualität als Patienten, die eine parodontale Behandlungsbedürftigkeit aufweisen.") nicht bestätigen. Allerdings zeigten sich klare negative Zusammenhänge zwischen mundgesundheitsbezogener Lebensqualität und der Anzahl an Parodontitis-Symptomen sowie Schmerzen im Mundbereich.

Der in Hypothese II angenommene Zusammenhang („Patienten ohne eine parodontale Behandlungsbedürftigkeit haben eine geringere Beeinträchtigung der krankheitsübergreifenden Lebensqualität als Patienten, die eine parodontale Behandlungsbedürftigkeit aufweisen.") ist abhängig von der Operationalisierung der krankheitsübergreifenden Lebensqualität. Einzelne Ergebnisse aus dem OHIP-G14 und dem allgemeinen Gesundheitsfragebogen sprechen für die Hypothese II. Bei Operationalisierung der Behandlungsbedürftigkeit über den PSI-Wert muss jedoch hinsichtlich des OHIP-G14 MLQ-Wertes, der SF-36 KSK und PSK sowie der vier Dimensionen des WHOQOL-BREF (physisch, psychisch, sozial, Umwelt) die Hypothese II verworfen werden.

Die Kovariaten Angst und Depression haben zwar (entgegen des in Hypothese III postulierten Zusammenhangs) keinen signifikanten Einfluss auf die mundgesundheitsbezogene Lebensqualität, wohl aber auf die übergreifende allgemeine Lebensqualität: Je höher die mittels HADS-D erhobenen Angstwerte, desto geringer die physische und psychische Lebensqualität und je höher die berichteten Depressionswerte, desto geringer die Werte in physischer, psychischer und sozialer Gesundheit. Die statistische Kontrolle der Kovariaten Alter führt zu einer Verschiebung der gefundenen signifikanten Zusammenhänge, leidet aber unter einer zu geringen Fallzahl. Bezüglich des Einflusses von Angst und Depression auf die mundgesundheitsbezogene Lebensqualität ist somit die Hypothese III nicht bestätigt.

Weitere Studien, die die multifaktorielle Gesamtheit der Lebensqualität erfassen, sind auf jeden Fall zu empfehlen und weiterhin notwendig. Wie in der Praxis beobachtet und von verschiedenen Autoren bestätigt, scheinen sich meist erst fortgeschrittene Stadien parodontaler Erkrankungen auf die Lebensqualität und mundgesundheitsbezogene 
Lebensqualität auszuwirken (Bernabé und Marcenes 2010; $\mathrm{Ng}$ und Leung 2006; Al Habashneh et al. 2012; Cunha-Cruz et al. 2007; Needleman et al. 2004). Dies hat zur Folge, dass ohne objektive Untersuchung in der zahnärztlichen Praxis Patienten erst sehr spät eine Parodontopathie bewusst bemerken. Umso wichtiger erscheinen Erkenntnisse darüber, ab wann und wie sich Parodontopathien auf die Lebensqualität auswirken, um die Patienten, aber auch das zahnmedizinische Personal für diese Erkrankungen zu sensibilisieren. Ein unbemerktes Fortschreiten der Erkrankung sollte vermieden werden, denn eine stark fortgeschrittene Parodontitis kann beträchtliche Auswirkungen auf die mundgesundheitsbezogene Lebensqualität haben (Needleman et al. 2004). Daneben bleibt die zielgerichtete regelmäßige Befundaufnahme des Zahn- und Parodontalbefundes zur Beurteilung der Erkrankungslast unumgänglich.

Obwohl in dieser Studie keine definitiven Aussagen über die Beeinträchtigung der mundgesundheitsbezogenen Lebensqualität durch eine parodontale Behandlungsbedürftigkeit getroffen werden können, sind dennoch bei multifaktorieller Betrachtung Zusammenhänge auf verschiedenen Ebenen wahrnehmbar, die durch weitere gesamtheitliche Untersuchungen überprüft werden sollten. 


\section{Zusammenfassung}

Die bisherigen Forschungsergebnisse zum Zusammenhang von Paradontitis und mundgesundheitsbezogener (MLQ) bzw. allgemeiner Lebensqualität zeichnen kein einheitliches Bild. Es fehlt bisher an einer gesamtheitlichen Untersuchung unter Verwendung unterschiedlicher Instrumente und somit Konzeptualisierungen von MLQ bzw. allgemeiner Lebensqualität und Parodontitis.

Die vorliegende Pilotstudie hatte das Ziel, als Machbarkeitsstudie Studienparameter für eine großangelegte Untersuchung, die die Auswirkungen einer parodontalen Behandlungsbedürftigkeit auf die mundgesundheitsbezogene und allgemeine (krankheitsübergreifende) Lebensqualität erforschen soll, zu überprüfen. Die die Lebensqualität mitbeeinflussenden Faktoren, wie bestimmte physische und psychische Erkrankungen (z. B. Depressionen, Diabetes mellitus) und orale Faktoren wie Karies, herausnehmbarer Zahnersatz und viele fehlende Zähne, sollten durch strenge Auswahlkriterien minimiert werden bzw. durch spezielle Fragen erkannt werden.

Hierzu wurden im Rahmen einer Pilotstudie 49 Patienten (34 Frauen, 15 Männer) im Alter von $49 \pm 9,7$ Jahren untersucht; das Mindestalter war auf 35 Jahre festgelegt. Neben dem zahnärztlichen Befund wurde der Parodontale Screening-Index (PSI) aufgenommen. Die Aufteilung in zwei Gruppen erfolgte anhand der PSI-Grade in eine Kontrollgruppe mit PSI 1-2 ohne parodontale Behandlungsbedürftigkeit (Gruppe A) und eine behandlungsbedürftige Gruppe mit PSI 3-4 (Gruppe B).

Die Erhebung Parodontitis-spezifischer Symptome erfolgte mit einer speziellen Checkliste. Für die MLQ wurde die Kurzversion des Oral Health Impact Profile (OHIP-G14) verwendet. Die subjektive Lebensqualität wurde mit dem Short-Form Health Survey (SF-36) und dem World Health Organization Quality of Life Scale (WHOQOL-BREF) ermittelt. Für die Erhebung von Angstzuständen und Depressionen wurde der Hospital Anxiety and Depression Scale (HADS-D) angewendet.

26 Patienten wiesen keine parodontale Behandlungsbedürftigkeit auf (Gruppe A: PSI 1-2) und 23 zeigten eine parodontale Behandlungsbedürttigkeit (Gruppe B: PSI 3-4). Der mittlere DMF-T-Wert war in beiden Gruppen signifikant unterschiedlich (A: 14,9 $\pm 4,4$; B: 17,7 $\pm 3,9$ ). Zwar waren keine signifikanten Unterschiede in der Einschätzung des eigenen Mundgesundheitszustands feststellbar, Gruppe A bewertete jedoch ihren allgemeinen Gesundheitszustand signifikant als besser (Gruppe A: 2,62 \pm 0,8, Gruppe B: 2,26 $\pm 0,62$ ). Patienten der Gruppe B berichteten signifikant häufiger über Schmerzen im Mundbereich, über Schlafprobleme, über hohen Blutzucker / Diabetes und insgesamt weniger Ruhe und Gelassenheit. Schmerzen im Mundbereich standen in engem Zusammenhang mit geringer 
mundgesundheitsbezogener Lebensqualität. Patienten, die über empfindliches Zahnfleisch berichteten, berichteten auch über geringere physische Lebensqualität. Je höher die Anzahl an vorhandenen Parodontitis-Symptomen, desto niedriger waren die mundgesundheitsbezogene, körperliche und physische Lebensqualität. Die vorliegende Studie konnte jedoch keine signifikaten Zusammenhänge von Gruppenzugehörigkeit und damit dem PSI-Grad und MLQ bzw. allgemeiner Lebensqualität aufzeigen. Zusammenhänge auf verschiedenen Ebenen sind aber erkennbar.

Ob sich diese Tendenzen weiter bestätigen lassen, müssen folgende Untersuchungen mit höheren Probandenzahlen und unter statistischer Kontrolle von Alter und Geschlecht zeigen. Die Studienparameter sollten für eine weitere Untersuchung überarbeitet werden, vor allem ist eine Reduzierung der Fragen für die Umsetzbarkeit in der Praxis unausweichlich. Auch die Gruppeneinteilung sollte weiter spezifiziert werden. 


\title{
$7 \quad$ Anhang
}

\section{$7.1 \quad$ Formulare}

\subsubsection{Ethikvotum}

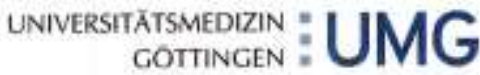

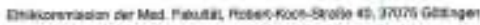 \\ Meczinische Feboultit \\ Varstzzender: Prof. Or. Jurgen Brockmaller \\ Herm \\ Prof. Dr. med. dent. Rainer F. Mausberg \\ Abt. Präventive Zahnmedizin. \\ Parodontologie und Kariologie \\ -im Hause - \\ Referentin \\ Regierungsratin Doris Wettschereck
0551/39-8644 Telefon \\ 37099 Göttingsn Briefpost \\ Rober-Koch-Straße 40, 37075 Göttingen \\ 0651/39-6629 Telefon \\ $0551 / 39-953 \%$ Fax \\ ethikermed.unt-goettingen.de E-Mail \\ www.ethididommission.med uni-goettingen.de \\ warab per Fax 22037 \\ 08.03.2012 br - se - gő Datum \\ Artragsnumener \\ studiontitel: \\ 2018/11 (bitte stets angeben) \\ Kiniscthe Untersuchung zur Eflassung der mundgesundheitsbezogenen \\ Lebensqualtat (MLQ) bei parodontal gesunden und erkrankten Erwachsenen \\ Antragstelier: \\ Prof. Dr. med. dent. Riainer F, Mausburg, Dr. med. Dent. Dirk Ziebhokz, Abt. \\ Prăventive Zghnmedizin. Parodontologe und Kariobogie, Pral. Dr. hum. biol. \\ Nicolo von Steinbüchet-Rheinwall, Prot. Dr. H. Schmidt. Abt. Medizinische \\ Pyychologie und Modizinische Soziologie, UMG \\ Folgande Untertagen warden zur Bewertung nachaemicht: \\ - Anschreiben nebet Erlautorungen vom 27.2.2012 \\ - Studienprotokoll vom 27.2.2012 \\ - informetionseschrift und Eimerstindriserklärung vom 27.2 .2012
}

Sehr geehrter Herr Prof. Dr. Mausterg, sehr geehrte Damen und Herren,

nach Ergänzung der 0.9 . Dokumente und Beantwortung der im vorlibufigen Votum aufgeführten Fragen in Ihrem Schreiben vom 27.2.2012 bestehen nunmehr keine ethischen und rechtichen Bedenken gegen die Durchtührung des oben genannten Forschungswortabens.

Wr wünschen thnen vioł Erfolg bei der Durchführung thres Projektes.

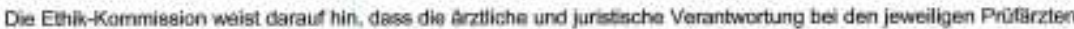
verbleibt.

Auf die Einhaitung einechlagiger Gesetze und Rechtsvorschritten wird hingowiesen. Die nach Rechitslage notwendigan Unterrichtungen (u. A. Anderum des Studienprotokolts, Maidıng von Zwischenfilien, neue Datenlage, Nachmeldung ven Prifentren, Abectilussbericht) sind der Ethik-Kommission unvorzìgich vorzulegen.

Die Ethik-Kammission bestatigh, dass sie auf Grundege nationaler Gesetze, Vorgchriften sowie der GCPACH-Richtinie arbeithet.

Mit freundlichen Grūßsen

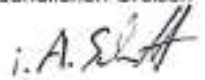

Prof. Dr. med. J. Brockmölier

Vorsitzender der Ethik-Kommission 


\subsubsection{Patientenaufklärung}

\section{UNIVERSITÄTSMEDIZIN $=\mathbf{J M G}$
GOTTINGEN $=\mathbf{M G}$}

\begin{tabular}{|ll|}
\hline Patientennummer: $\ldots \ldots$ & Datum: $\ldots+\cdots, \cdot \ldots$ \\
Geburtsdatum: & Praxiscode: $\ldots \ldots+\cdots$ \\
\hline
\end{tabular}

Dr. med. dent, Dirk Zebolz, MSc,; Universitatsmedizin Guttingen, Zentrum Zahn-, Mund- und Kieferheilkunde; Abteilung Pránentive Zahnmedizin, Parodontologie und Kariologie; Robert-Koch-Str. 40, 37075 Gottingen; Tel.: 0551/3922877

\section{Patientenaufklărung}

.Klinische Untersuchung zur Erfassung der mundgesundheitsbezogenen Lebensqualitat bei parodontal gesunden und erkrankten Enwactisenen

SEHR GEEHRTE PATENTIN, SEHR GEEHRTER PATIENTI

Wir bitten um treiwillige Teilnahme an unserer Studie: .Klinische Untersuchung zur Erfassung der mundgesundheitsbezogenen Lebensqualităt bei parodontal gesunden und erkrankten Erwachsenen"

Der Begriff ,Mundgesundheitsgezogene Lebensqualitat" beschreibt, wie wir unsere Mundgesundheit selber erleben. Dabel sind die selbsterlebte allgemeine Gesundheit wie auch Mundgesundheit wesentliche Bestandteile der eigenen Lebensqualitat und des personlichen Wohlbefindens.

Einschränkungen in der Mundgesundheit konnen sich ungünstig auf das Allgemeinbefinden und somit auch auf die Lebensqualitat auswiken. Die Mundgesundheit bzw. Krankheitszustande im Mund, wie z.B. des Zahnes (Karies) und des Zahnhalteapparates (Parodontitis) lassen sich objektiv durch Erfassung von zahnärtlichen Befunden beschreiben. Das Befinden des Patienten ist jedoch nicht immer deckungsgleich mit dem Befund des Zahnarztes. Deshalb wird mit Hilfe eines speziellen Fragebogens (.Oral Health impact Profile (OHIP)" versucht, einen Einblick in die selber vom Patienten empfundene Mundgesundheit $z \mathrm{U}$ bekommen. Eine klinische Bewertung durch den behandelnden Zahnarzt zusammen mit dem eigenen Empfinden desir Patienten/in erlauben eine ganzheitliche Betrachtung der mundgesundheitsbezogenen Lebensqualitat des Menschen.

Die Bedeutung solcher Forschungsergebnisse über die mundgesundheitsbezogene Lebensqualităt ist vielschichtig und kann z,B. im klinischen Alltag in der Entscheidungsfindung bei unterschiedlichen Therapievarianten, in der Qualitătssicherung und der Forschung im Offentlichen Gesundheitswesen und für dessen Weiterentwicklung eingesetzt werden.

Darūber hinaus müssen verschiedene andere Einflussfaktoren, die über thre empfundene Mundgesundheit hinausgehen und beeinflussen kōnnen, berūcksichtigt werden. Dazu gehören die allgemeine Lebensqualitāt, Angstlichkeit und Depression, soziodemographische Daten und thr allgemeiner Gesundheitszustand.

Wir suchen Grūnde dafür, in welchem Maße Erkrankungen des Zahnhalteapparates (besteht aus Zahnfleisch und Knochen), Z.B. Zahnffeischentzündung oder Parodontitis, einen Einfluss auf die Lebensqualităt haben. Mit threr Teilnahme können Sie uns helfen herauszufinden, in welchem Umfang ein solcher Einfluss besteht. Im Rahmen dieser klinischen Untersuchung sind daher die Erfassung und der 


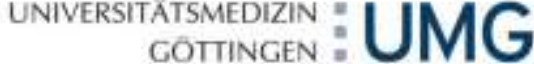

Vergleich der wahrgenommenen Beeinträchtigungen der Mundgesundheit und subjektiven allgemeinen Lebensqualitat durch den Patienten selbst und der objektiven klinischen Bewertung mit Hilfe klinischer Befunde (Sanierungs - und Versorgungsgrad und Situation des Zahnhalteapparates) durch den Zahnarzt geplant.

Um Aussagen über den jeweiligen Zustand ihres Zahnhalteapparates treffen zu können, wird ein ausfuhricher zahnarztlicher Befund erhoben. Die Unters uchung wird von ihrem Hauszahnarzt durchgefuhrt und ist aberwiegend schmerzfrei / kaum belastend. Des Weiteren bitten wir Sie den Oral Health impact Profile (OHIP) - Fragebogen sowie einige erganzende Fragebogen (Sf-36, WHOQOL-Bref, HAD-D. Gesundheitszustand, soziodemographische Daten) zu beantworten.

Der Aufwand an Zeit pro Untersuchung betragt ca. 30 bis 45 Minuten; es entstehen fur Sie keine zusatzlichen Kosten. Risiken und Nebenwirkungen bei der Durchfuhrung der Untersuchung sind nicht zu erwarten bzw. bestehen nicht.

Thre personenbezogenen Daten unterliegen dem Datenschutz und werden vom Leiter der Prüfung nicht weitergegeben. Sie werden pseudonymisiert behandelt, d.h. Verschlisselung von Daten ohne Namensnennung und Nummemcodierung. Die Zuordnung der Daten zu eìner Person ist nur möglich, wenn hierfur der Schlissel eingesetzt wird, mit dern die Daten pseudonymisiert wurden. Die pers onenbezogenen Daten werden unter besonderen Schutzvorkehrungen getrennt von den pseudonymisierten Daten aufbewahrt: Eine Entschlüsselung ist nur durch die verantwortlichen Studienărzte möglich; Dritte erhalten keinen Einblick in die Originalunterlagen. Im Rahmen der Studie werden Ihre personenbezogenen Daten mit studienspezifschen Erfassungsbögen erhoben und pseudonymisiert in eine Studiendatenbank übertragen. Auf die Daten haben nur der Leiter der Prüfung und die Prüarzte Zugriff, die Daten sind durch ein Passwort gesichert. Die Prüfboggen (Erfassungsbōgen) werden in einem Prüfordner gesammelt und beim Leiter der Prufung fur 10 Jahre aufbewahrt. Die gespeicherten Daten werden nur zu Untersuchungszwecken verwendet und nach der Auswertung vemichtet. Darüber hinaus erhät lhr behandeinder Zahnarzt keine Angaben zu den Ergebnissen / Erkenntnissen der mit den Fragebögen erhobenen Daten.

Sie haben die Möglichkeit, đurch Angabe auf der Einwilligungserklärung, um Information bezüglich auffalliger psychischer Probleme zu bitten. Unsere Studienzentrale wird sich entsprechend mit thnen in Verbindung setzen und ggf. ein indiviuelles Hilsangebot mit mõglichen Anlaufstellen anbieten (falls informationen erbeten).

Wir bitten um thre freiwiltige Teilnahme an der Studie. Sie kōnnen jederzeit die Teilnahme widemufen, ohne Angabe von Grunden und ohne Nachteile erwarten zu mussen. Nach Ihrem Widerruf erfolgt unverzuglich die Vemichtung lhrer personenbezogenen Daten.

Für Ruckfragen stehen thnen die Studienleiter unter folgenden Telefonnummem 0551 - 398368 zur Vertuggung

Vielen Dank fur Ihre Teilnahme.

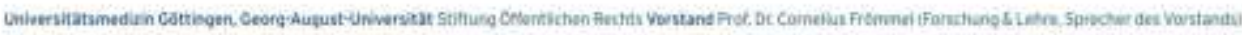

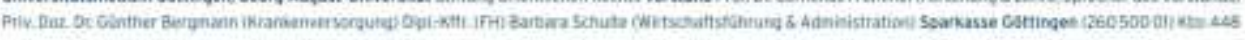




\subsubsection{Patienteneinwilligung}

\section{UNIVERSITÄTSMEDIZIN $=\mathbf{W M G}$
GOTTINGEN $=\mathbf{M G}$}

\begin{tabular}{|ll|}
\hline Patientennummer: $\ldots \ldots$ & Datum: $\ldots \ldots \ldots$ \\
Geburtsdatum: & Praxiscode: $\ldots \ldots$
\end{tabular}

Dr. med. dent. Dirk Zebolz, MSc.; Universitatsmedizin Götingen, Zentrum Zahn-, Mund- und Kieferheilkunde; Abteilung Präventive Zahnmedizin, Parodontologie und Kariologie; Robert-Koch-Str. 40, 37075 Gottingen; Tel.: 0551/3922877

\section{Einwilligungserklärung}

Klinische Untersuchung zur Erfassung der mundgesundheitsbezogenen Lebensqualitat bei parodontal gesunden und erkrankten Erwachsenen"

Ich, wurde wo meinem Arzt vollstăndig über Wesen,

Bedeutung und Tragweite der klinischen Untersuchung mit dem Ttel:

„Klinische Untersuchung zur Erfassung der mundgesundheitsbezogenen Lebensqualitat bei parodontal gesunden und erkrankten Erwachsenen"

aufgeklart.

Mir ist bekannt, dass im Rahmen dieses Forschungsvorhabens personenbezogene Daten erhoben und in pseudonymisierter (verschlusselter) Form aufgezeichnet und ge speichert werden. Die Daten sollen fur einen Zeitraum von 10 Jahren aufbewahrt werden, danach werden alle personenbezogenen Daten geloscht. Die personenbezogenen Daten werden nicht an Dritte weitergegeben.

Ich weiß, dass ich mein Einverstandnis zur Speicherung der personenbezogenen Daten jederzeit widerrufen kann. Im Falle des Widerrufs werden alle pe rsonenbezogenen Daten geloscht.

Ich hatte ausreichend Zeit, mich zur Teilnahme an dieser Untersuchung zu entscheiden und weiB, dass die Teilnahme freimilig ist. Alle Fragen wurden zu meiner Zufriedenheit beantwortet.

Mir ist bekannt, dass ich jederzeit und ohne Angaben von Grunden diese Zustimmung widerrufen kann, ohne dass sich dieser Entschluss nachteilig auf meine weitere Behandlung auswirkt.

Ich habe eine Kopie der Patienteninformation und dieser Einwilligungserklarung erthalten, Ich erklare hiernit meine freiwilige Teilnahme an dieser klinischen Studie.

Ich mõchte bei Verdacht auf schwerwiegende psychische Probleme informiert werden! Ja () Nein ()

Ort und Datum

Unterschritt des Teilnehmers

Ort und Datum

Unterschrie des Prüfarztes 


\subsubsection{Zahnärztlicher Untersuchungsbogen}

\section{UNIVERSITÁTSMEDIZIN $=1 \mathrm{MG}$}

\begin{tabular}{|ll|}
\hline Patientennummer: $\ldots \ldots$ & Datum: $\ldots \ldots+\ldots,--$ \\
Geburtsdatum: & Praxiscode: $\ldots \ldots-\ldots$ \\
\hline
\end{tabular}

\section{Zahnärztlicher Untersuchungsbogen}

\section{Zahnärztlicher Befund:}

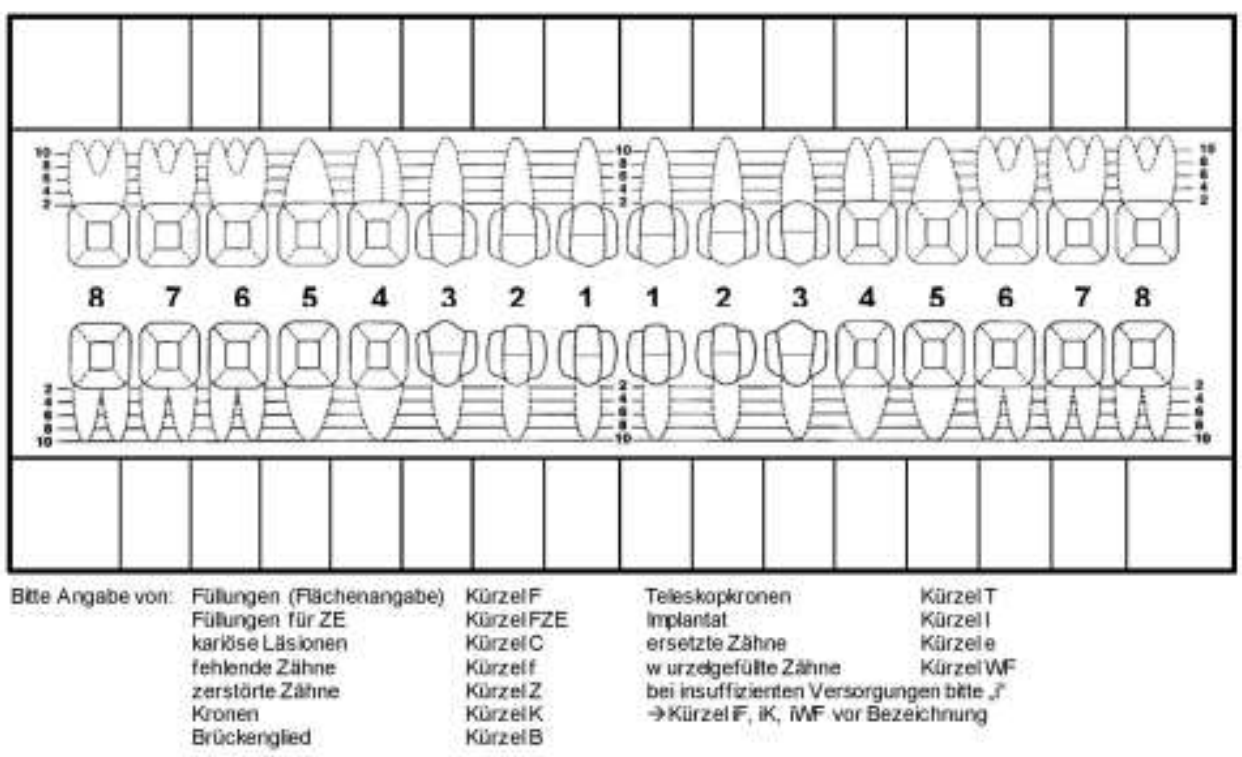

PS1:

\begin{tabular}{|l|l|l|}
\hline I.Sextant & 2Sextant & 3.Sextant \\
\hline 6.Sextant & 5.Sextant & 4.Sextant \\
& & \\
\hline
\end{tabular}

Grad 0: Farbige Mbrkierung volstandigs schttbar, keh Bluten aut Sandieren, kein Zahnstein, keine marginaken britatone Grad 1: Farbiga Merberung volstandig sichtbar, Butung auf Eondenen, kein Zahnstein, keine marginalen kritationen

Grad 2: Farbigs Markerung volstando sichtbar, Zahnsien undibder iatrogene marginabe rreationan

Grad 3: Fartioe Markeerung nur noch leilw eisessichthar (Sonderungstefe $3,5.5,5 \mathrm{~mm}$ )

Grad4: Farbioe Markierung nichit mehr sichitbar (Sondierungstiefe uber 5,5 mms

Wurde schon einmal eine Parodontitisbehandlung durchgefuhrt? $\square$ Nein $\square$ Ja,wann 


\subsubsection{Parodontitis-Checkliste (Pcheck-12)}

UNIVERSITÁTSMEDIZIN GOTTINGEN

\begin{tabular}{|ll|}
\hline Patientennummer: $\ldots+-$ & Datum: $\ldots \ldots+\cdots,--$ \\
Geburtsdatum: & Praxiscode: $\ldots+\cdots$ \\
\hline
\end{tabular}

\section{Parodontitis Checkliste}

Bitte beantworten Sie, ob sie in den vergangenen 12 Monaten folgende Symptome an sich wahrgenommen haben:

\begin{tabular}{|c|c|c|}
\hline Symptom & Ja & Nein \\
\hline \multicolumn{3}{|l|}{ Zahnfle sischschwellung } \\
\hline \multicolumn{3}{|l|}{ schmerzendes Zahnfleisch } \\
\hline \multicolumn{3}{|l|}{ empfindliches Zahnfeisch } \\
\hline \multicolumn{3}{|l|}{ Zahnfle ischbluten } \\
\hline \multicolumn{3}{|c|}{$\begin{array}{l}\text { zurückgehendes Zahnfleisch (Rezession, } \\
\text { freiliegende Wurzeloberfläiche) }\end{array}$} \\
\hline \multicolumn{3}{|l|}{$\begin{array}{l}\text { Zahnlberempfindlichkeit } \\
\text { (Heil/Kalt bzw. süß/sauer) }\end{array}$} \\
\hline \multicolumn{3}{|l|}{ Zahnkckerung } \\
\hline \multicolumn{3}{|l|}{$\begin{array}{l}\text { Zahnwanderung / Veränderung der } \\
\text { Zahnstellung }\end{array}$} \\
\hline \multicolumn{3}{|l|}{ Veränderung des Bisses } \\
\hline \multicolumn{3}{|l|}{ Mundgeruch } \\
\hline \multicolumn{3}{|l|}{ schlechter Geschmack } \\
\hline \multicolumn{3}{|l|}{ Zahnschmerzen } \\
\hline \multicolumn{3}{|l|}{$\begin{array}{l}\text { vorab Parodontalbehandlung / } \\
\text { Taschenbehandlung }\end{array}$} \\
\hline \multicolumn{3}{|l|}{$\begin{array}{l}\text { regelmüBige/routinemilBige } \\
\text { Kontrolluntersuchung }\end{array}$} \\
\hline \multicolumn{3}{|l|}{$\begin{array}{l}\text { beschwerde- bzw, schmerzbedingte } \\
\text { Kontrolluntersuchung }\end{array}$} \\
\hline $\begin{array}{l}\text { regelmïBige Durchfihrung einer } \\
\text { professionelle Zahnreinigung ( } 1-2 \\
\text { jährlich }\end{array}$ & & \\
\hline
\end{tabular}




\subsubsection{Oral Health Impact Profile (OHIP-G14)}

\section{UNIVERSITÁTSMEDIZIN $=1 \mathbf{M G}$
GOUTTINGEN $=0$}

\begin{tabular}{|ll|}
\hline Patientennummer: $\ldots \ldots$ & Datum: $\ldots \ldots-\ldots,-$ \\
Geburtsdatum: & Praxiscode: $\ldots \ldots-\ldots$ \\
\hline
\end{tabular}

OHIP - G14

(John et al. 2004)

Zutreffendes bitte ankreuzen:

\begin{tabular}{|l|c|c|c|c|c|}
\hline Wie würden Sie Ihren... & Ausgezeichnet & Sehr gut & gut & mitreimaBig & schlecht \\
\hline ....allgemeinen Ges undheitszustand einschatzen? & & & & & \\
\hline ...Mundgesundheits zustand einschaitzen? & & & & & \\
\hline
\end{tabular}

\begin{tabular}{|l|l|l|l|l|l|}
\hline $\begin{array}{l}\text { Hatten Sie im vergangenen Monat aufgrund von Problemen mit lhren Zahnen, im } \\
\text { Mundbereich oder mit ihrem Zahnersatz... }\end{array}$ & $\begin{array}{c}\text { Sehr } \\
\text { oft }\end{array}$ & \multicolumn{1}{|c|}{ of } & $\begin{array}{c}\text { Ab } \\
\text { und } \\
\text { zal }\end{array}$ & kaum & nie \\
\hline Schwierigkeiten bestimmmte Worte aus zusprechen? & & & & & \\
\hline das Gefuht, ihr Geschmackssinn war beeinträchtigt? & & & & & \\
\hline den Eindruck, dass IthrLeben ganz alligemein weniger zuffiedenstellend war? & & & & & \\
\hline Schwierigkeiten zu entspannen? & & & & & \\
\hline
\end{tabular}

\begin{tabular}{|c|c|c|c|c|c|}
\hline $\begin{array}{l}\text { Ist es im vergangenen Monat aufgrund von Problemen mit lhren Zahnen, im } \\
\text { Mundbereich oder mit Ihrem Zahnersatz vorgekommen,... }\end{array}$ & $\begin{array}{c}\text { Sehr } \\
\text { of }\end{array}$ & of & $\begin{array}{l}\text { Ab } \\
\text { und } \\
\text { zu }\end{array}$ & kaum & nie \\
\hline \multicolumn{6}{|l|}{ dass Sie sich anges pannt gefuhithaben? } \\
\hline \multicolumn{6}{|l|}{ dass Sie Ihre Mahlzenten unterbrechen mus sten? } \\
\hline \multicolumn{6}{|l|}{ dass es ithnen unangenehm war, bestimmte Nahrungsmittel zu essen? } \\
\hline \multicolumn{6}{|l|}{ dass Sie anderen Mens chen gegenūbereher reizbargewesen sind? } \\
\hline \multicolumn{6}{|l|}{ dass es thnen schwer getallen ist, thren altăglichen Beschattigungen nachzugehen? } \\
\hline \multicolumn{6}{|l|}{ dass Sie vollikommen unfahig waren, etwas zi tun? } \\
\hline \multicolumn{6}{|l|}{ dass Sie sich ein wenig wrlegen gefuhithaben? } \\
\hline đass Ithre Emährung unbefriedigend gewesen ist? & & & & & \\
\hline
\end{tabular}

\begin{tabular}{|l|c|c|c|c|c|}
\hline Hatten Sie im verqangenen Monat... & $\begin{array}{c}\text { Sehr } \\
\text { of }\end{array}$ & oft & $\begin{array}{c}\text { Ab } \\
\text { und } \\
\text { zul }\end{array}$ & kaum & nie \\
\hline Schmerzen im Mundbereich? & & & & & \\
\hline $\begin{array}{l}\text { ein Gefuhl der Unsicherhedin Zus ammenhang mit Ihren Zahinen, lhrem Murld oder lhrem } \\
\text { Zahners atz? }\end{array}$ & & & & & \\
\hline
\end{tabular}




\subsubsection{Allgemeiner Gesundheitsfragebogen}

\section{UNIVERSITÁTSMEDIZIN $=\mathbf{W M G}$
GÓTTINGEN $=\bigcup \mathbf{M G}$}

\begin{tabular}{|ll|}
\hline Patientennummer: $\ldots \ldots$ & Datum: $\ldots \ldots+\ldots,--$ \\
Geburtsdatum: & Praxiscode: $\ldots \ldots-\ldots$ \\
\hline
\end{tabular}

Ihr allgemeiner Gesundheitszustand

(Steinbüchel et al, 2010)

Haben Sie trres Wissens nach eine der folgenden Gesundheitszustände oder Probleme?

\begin{tabular}{|c|c|c|}
\hline \multirow{3}{*}{$\begin{array}{l}\text { Allergien (Heuschnupfen, chronische Nebenhöhlenprobleme und andere } \\
\text { Asthma oder andere ernst zunehmende Lungenprobleme, z.8. chronische Bronchitis. } \\
\text { Lungenemphysem) }\end{array}$} & \multicolumn{2}{|c|}{$\begin{array}{c}\text { Bitte kreisen Sie Ihre } \\
\text { Antwort ein }\end{array}$} \\
\hline & Nein & Ja \\
\hline & Nein & Ja \\
\hline Geruchs- oder Geschmacksprobleme .......... & Nein & da \\
\hline Sehprobieme (auch mit Brille oder Kontaktinsen) & Nein & da \\
\hline Hôrprobleme (auch mit Hörgerat) & Nein & Ja \\
\hline Schilddrusenprobleme & Nein & Ja \\
\hline Hoher Blutzucker oder Diabetes & Nein & $\mathrm{J}_{3}$ \\
\hline Schlafprobleme & Nein & Ja \\
\hline Koptschmerzen oder Migrane & Nein & $\mathrm{Ja}$ \\
\hline Nervosinat .............. & Nein & Ja \\
\hline Depression & Nein & Ja \\
\hline Mangel an Energie & Nein & da \\
\hline Mangel an Kraft . & Nein & Ja \\
\hline Rückenprobleme (inklusive Bandscheibe oder Rückgrat) & Nein & Ja \\
\hline Arthritis & Nein & $\mathrm{Ja}$ \\
\hline Bewegungsschwierigkelten, verursacht durch eine Schadelhirnverletzung & Nein & Ja \\
\hline $\begin{array}{l}\text { Bewegungsschwierigkeiten, verursacht durch anderer Probleme als die } \\
\text { Schade/himverletzung }\end{array}$ & Nein & Ja \\
\hline $\begin{array}{l}\text { Größere Lahmungen oder Nenenschmerzen (inkiusive Schlaganfall, Multiple } \\
\text { Sklerose, Muskelschwund, Nenenprobieme), verursacht durch eine } \\
\text { Schädelhimverletzung }\end{array}$ & Nein & Ja \\
\hline
\end{tabular}




\section{unmasamamanas UMG}

Größere Lähmungen oder Nenenschmerzen (inklusive Schlaganfall, Multiple Sklerose, Muskelschwund, Nervenprobleme), verursacht durch andere Probleme als eine Schädelhimverletzung

Amputation eines Ams oder Beins

Hoher Blutdruck oder Bluthochdruck

Herzfehler oder Herz vergrößerung

Angina

Herzattacke, Myokardinfarkt (MI) oder Herzinfarkt

Gebrauch eines Herzschrittmachers

Chronische Damentzündung, Kolitis

Geschwär (Zwolffingerdamgeschwar oder Magengeschwlin)

Nierenkrankheit

Krabs (innerhalb der letzten drei Jahre diagnostiziert)

Andere schwerwiegende Probleme (Bitte angeben)

\begin{tabular}{|c|c|}
\hline Nein & Ja \\
\hline Nein & Ja \\
\hline Nein & Ja \\
\hline Nein & Ja \\
\hline Nein & Ja \\
\hline Nein & Ja \\
\hline Nein & Ja \\
\hline Nein & Ja \\
\hline Nein & Ja \\
\hline Nein & Ja \\
\hline Nein & Ja \\
\hline Nein & Ja \\
\hline
\end{tabular}




\subsubsection{Short-Form Health Survey (SF-36)}

\section{Fragebogen zum Allgemeinen Gesundheitszustand} (SF - 36)

Selbstbeuteilungsbogen

Zeitfenster 4 Wochen

In diesem Fragebogen geht es um die Beurteilung thres Gesundheltszustandes, Der Bogen ermóglicht es, im Zeitverlauf nachzuvoltziehen, wie Sie sich fihien und we Sie im Alltag zurechtkommen.

Bitte beantworten Sie jede der Fragen, indem Sie bei den Antwortmbglichkeiten die Zahl ankreuzen, die am besten auf Sie zutrim.

\begin{tabular}{|l|c|c|c|c|c|}
\hline 1. We würden Sie itren Gesundheitszustand & $\begin{array}{c}\text { Ausge- } \\
\text { zeichnet }\end{array}$ & Sehr gut & Gut & Weniger gut & Schlecht \\
im allgemeinen beschreiben? & 1 & 2 & 3 & 4 & 5 \\
\hline
\end{tabular}

\begin{tabular}{|l|c|c|c|c|c|}
\hline 2. Im Vergieich zum vergangenen Jaht, wie & $\begin{array}{c}\text { Derzeit } \\
\text { vel } \\
\text { besser } \\
\text { wurden Sie Itren dergeitigen } \\
\text { Gesundheitszustand beschreben? }\end{array}$ & $\begin{array}{c}\text { Derzeil } \\
\text { etwas } \\
\text { besser }\end{array}$ & $\begin{array}{c}\text { Etwa wie vor } \\
\text { einem Jahr }\end{array}$ & $\begin{array}{c}\text { Derzeit etwas } \\
\text { schilechter }\end{array}$ & $\begin{array}{c}\text { Derzeit } \\
\text { vel } \\
\text { schlechter } \\
5\end{array}$ \\
\hline
\end{tabular}

\begin{tabular}{|c|c|c|c|c|}
\hline 3. & $\begin{array}{l}\text { Im folgenden sind einige Tatigkeiten } \\
\text { beschrieben, die Sie velleicht an } \\
\text { einem normalen Tag aus üben. } \\
\text { Sind Sie durch thren derzeifigen Gesundhels- } \\
\text { zustand beidiesen Tatigkeiten eingeschvanind? } \\
\text { Wann ja, wie stark? }\end{array}$ & $\begin{array}{l}\text { Ja, stark } \\
\text { eingeschrarkt }\end{array}$ & $\begin{array}{l}\text { Ja, etwas } \\
\text { eingeschränkt }\end{array}$ & $\begin{array}{l}\text { Nein, uberhaupt } \\
\text { nicht eingeschrankt }\end{array}$ \\
\hline 3.a & $\begin{array}{l}\text { anstrengende Tatigkeiten, } 2 \text { B. schnell } \\
\text { laufen, schwere Gegenstande heben, } \\
\text { anstrengenden Sport treiben }\end{array}$ & 1 & 2 & 3 \\
\hline 3.b & $\begin{array}{l}\text { mittelschwere Tatigkeiten, } \mathrm{zB} \text {. einen Tisch } \\
\text { vers chieben, staubsaugen, kegeln, } \\
\text { Golfspielen }\end{array}$ & 1 & 2 & 3 \\
\hline 3.c & Einkauts taschen heben und tragen & 1 & 2 & 3 \\
\hline 3.d & mehrere Treppenabsătze steigen & 1 & 2 & 3 \\
\hline 3.6 & einen Treppenabsatzsteigen & 1 & 2 & 3 \\
\hline $3 . f$ & sich beugen, knien, bûcken & 1 & 2 & 3 \\
\hline 3.9 & mehr als 1 Kilometer $z u$ Fuß gehen & 1 & 2 & 3 \\
\hline & $\begin{array}{l}\text { mehrere Straßenkreuzungen weit } \\
\text { zu Fuß gehen }\end{array}$ & 1 & 2 & 3 \\
\hline 3.1 & eine Straßenkreuzung weitzu Fuß gehen & 1 & 2 & 3 \\
\hline
\end{tabular}




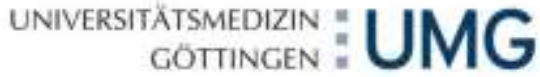

\begin{tabular}{|l|l|l|l|}
\hline 3.j sich baden oder anziehen & 1 & 2 & 3 \\
\hline
\end{tabular}

\begin{tabular}{|c|c|c|c|}
\hline & 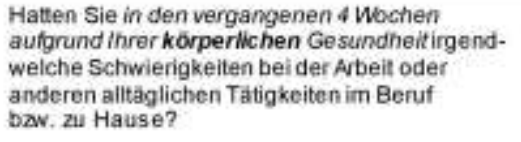 & Ja & Nein \\
\hline 4.a & lch konnte nichtso lange wie ublich tatig sein & 1 & 2 \\
\hline 4.b & ich habe weniger geschafft als ich wollte & 1 & 2 \\
\hline 4.0 & Ich konnte nur bestimmte Dinge tun & 1 & 2 \\
\hline & ich hatte Schwierigkeiten bei der Aus fuhrung & 1 & 2 \\
\hline
\end{tabular}

\begin{tabular}{|c|c|c|c|}
\hline & 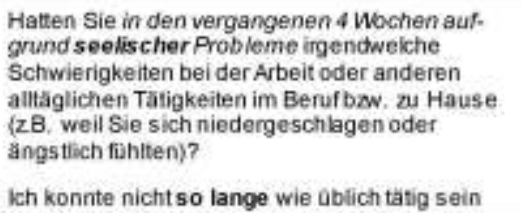 & Ja & Nein \\
\hline 5.b & ich habe weniger geschafft als ich wollte & 1 & 2 \\
\hline & kch konnte nichtso sorgfaltig wie ublich arbeiten & 1 & 2 \\
\hline
\end{tabular}

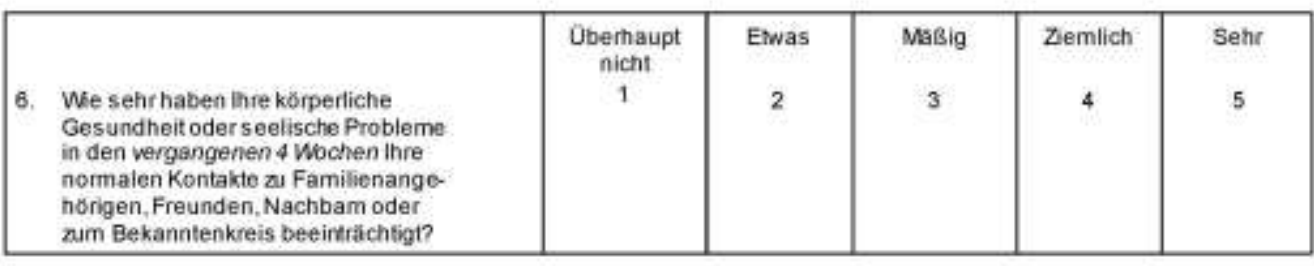

\begin{tabular}{|c|c|c|c|c|c|c|}
\hline 7. We stark waren thre Schmerzen in & $\begin{array}{c}\text { Keine } \\
\text { Schmerzen } \\
1\end{array}$ & $\begin{array}{c}\text { Sehr leicht } \\
2\end{array}$ & $\begin{array}{c}\text { Leicht } \\
3\end{array}$ & $\begin{array}{c}\text { Narig } \\
4\end{array}$ & $\begin{array}{l}\text { Stark } \\
5\end{array}$ & $\begin{array}{l}\text { Sehr } \\
\text { stark } \\
6\end{array}$ \\
\hline den vergangenen 4 Wochen? & & & & & & \\
\hline
\end{tabular}

\begin{tabular}{|c|c|c|c|c|c|c|}
\hline & & $\begin{array}{l}\text { Oberhaupt } \\
\text { nicht }\end{array}$ & Etwas & Maßig & Zemlich & Sehr \\
\hline 8. & $\begin{array}{l}\text { Inwieweithaben die Schmerzen Sie } \\
\text { in den wergangenen } 4 \text { Wochen bei } \\
\text { der Aus Abung Ihrer Alltagstatigkeiten } \\
\text { Zu Hause und im Berufbehindert? }\end{array}$ & 1 & 2 & 3 & 4 & 5 \\
\hline
\end{tabular}
a. Hause und im Benfbehindert?

\begin{tabular}{|l|l|l|l|l|l|l|}
\hline $\begin{array}{l}\text { In diesen Fragengehtes darum, wie } \\
\text { Sie sich fuhlen und wie es lhnen } \\
\text { in den vergangenen 4 Wochen gegangen ist. }\end{array}$ & Immer & Meistens & Zemlich of & Manchmal & Selten & Nie \\
(Bitte kreuzen Sie in jeder
\end{tabular}




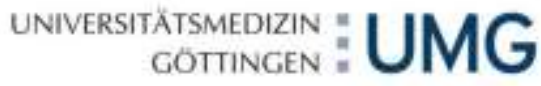

\begin{tabular}{|c|c|c|c|c|c|c|c|}
\hline & $\begin{array}{l}\text { Zeile die Zahi an, die threm Befinden } \\
\text { am ehesten entspricht.) } \\
\text { We ot waren Sie in den } \\
\text { vergangenen } 4 \text { Wochen }\end{array}$ & & & & & & \\
\hline 9.9 & ... voller Schwung? & 1 & 2 & 3 & 4 & 5 & 6 \\
\hline $9 . b$ & ... sehr nervis? & 1 & 2 & 3 & 4 & 5 & 6 \\
\hline $9 . \mathrm{c}$ & $\begin{array}{l}\text { so niederges chlagen, dass Sie } \\
\text { nichts aufheitern konnte? }\end{array}$ & 1 & 2 & 3 & 4 & 5 & 6 \\
\hline 9.d & ... ruhig und gelassen? & 1 & 2 & 3 & 4 & 5 & 6 \\
\hline $9 . \mathrm{e}$ & ... voller Energie? & 1 & 2 & 3 & 4 & 5 & 6 \\
\hline 9.1 & ... entmutigt und traurig? & 1 & 2 & 3 & 4 & 5 & 6 \\
\hline 9.9 & ... ersch bptt? & 1 & 2 & 3 & 4 & 5 & 6 \\
\hline $9 . \mathrm{h}$ & ... gluicklich? & 1 & 2 & 3 & 4 & 5 & 6 \\
\hline $9 . i$ & mũde? & 1 & 2 & 3 & 4 & 5 & 6 \\
\hline
\end{tabular}

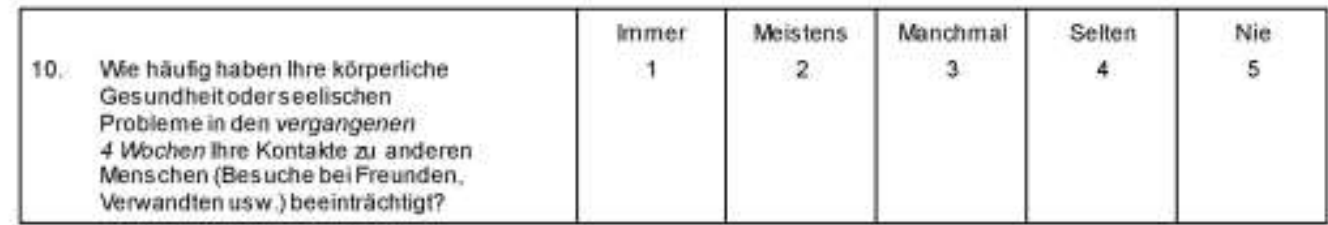

\begin{tabular}{|c|c|c|c|c|c|c|}
\hline & $\begin{array}{l}\text { inwieweit trift jede der folgenden } \\
\text { Auss agen aufSie zu? }\end{array}$ & $\begin{array}{l}\text { trift } \\
\text { ganz } \\
\text { au }\end{array}$ & $\begin{array}{l}\text { trim weit- } \\
\text { gehend zu }\end{array}$ & $\begin{array}{l}\text { weik } \\
\text { nicht }\end{array}$ & $\begin{array}{l}\text { trift weit- } \\
\text { gehend } \\
\text { nicht zu }\end{array}$ & $\begin{array}{l}\text { trith } \\
\text { überhaupt } \\
\text { nicht zu }\end{array}$ \\
\hline 11.a & $\begin{array}{l}\text { Ich scheine etwas leichter als } \\
\text { andere krank zu werden }\end{array}$ & 1 & 2 & 3 & 4 & 5 \\
\hline $11 . b$ & $\begin{array}{l}\text { Ich bin genauso gesund wie alle } \\
\text { anderen, die ich kenne }\end{array}$ & 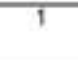 & 2 & 3 & 4 & 5 \\
\hline 11.0 & $\begin{array}{l}\text { Ich erwarte, dass meine Gesundhet } \\
\text { nachilasst }\end{array}$ & 1 & 2 & 3 & 4 & 5 \\
\hline 11.d & $\begin{array}{l}\text { Ich entreue mich ausgezeichneter } \\
\text { Gesundheit }\end{array}$ & 1 & 2 & 3 & 4 & 5 \\
\hline
\end{tabular}




\subsubsection{World Health Organization Quality of Life Scale (WHOQOL- BREF)}

\section{UNIVERSITÄTSMEDIZIN $: \mathbf{M M G}$
GÖTTINGEN $: \mathbf{M G}$}

\begin{tabular}{|ll|}
\hline Patientennummer: $\ldots+-$ & Datum: $\ldots \ldots+-,--$ \\
Geburtsdatum: & Praxiscode: $\ldots \ldots-\ldots$ \\
\hline
\end{tabular}

WHOQOL-BREF

(Angermeyer et at, 2000)

Sind Sie gegenwärtig krank? $\square$ ja $\square$ nein

Wenn ja, was ist thre Dlagnose?

Bitte lesen Sie jede Frage, uberlegen Sie, wie Sie sich in den vergangenen zwei Wochen gefuhlt haben, und kreuzen Sie die Zahl auf der Skala an, die fur Sie am ehesten zutrifft.

\begin{tabular}{|l|l|c|c|c|c|c|}
\hline & & $\begin{array}{c}\text { Sehr } \\
\text { schlecht }\end{array}$ & schlecht & $\begin{array}{c}\text { Muttel- } \\
\text { mäsig }\end{array}$ & Gut & Sehr gut \\
\hline 1 & We wüden Sie thre Lebensqualitat beunteilen? & 1 & 2 & 3 & 4 & 5 \\
\hline 2 & We zufrieden sind Sie mitthrer Gesundheit? & 1 & 2 & 3 & 4 & 5 \\
\hline
\end{tabular}

In den folgenden Fragen geht es darum, wie stark Sie wahrend der vergangenen zwei Wochen bestimmte Dinge erlebt haben 


\section{UNIVERSITÄTSMEDIZIN $=\mathbf{M M G}$
GOTTIINGEN $=\bigcup \mathbf{M G}$}

\begin{tabular}{|c|c|c|c|c|c|c|}
\hline & & $\begin{array}{l}\text { Uberhaupt } \\
\text { nicht }\end{array}$ & Ein wenig & $\begin{array}{l}\text { Mittel- } \\
\text { matigig }\end{array}$ & Zemlich & Außerst \\
\hline 3 & $\begin{array}{l}\text { We stark weden Sie durch Schmerzen daran } \\
\text { gehindert, notwendige Dinge zu tun? }\end{array}$ & 1 & 2 & 3 & 4 & 5 \\
\hline 4 & $\begin{array}{l}\text { Wie sehr sind Sie auf medizin ische Behandlung } \\
\text { angewiesen, um das tägliche Leben zu meistem? }\end{array}$ & 1 & 2 & 3 & 4 & 5 \\
\hline 5 & We gut kðnnen Sie Ihr Leben genießen? & 1 & 2 & 3 & 4 & 5 \\
\hline 6 & Betrachten Sie In Leben als sinnoti? & 1 & 2 & 3 & 4 & 5 \\
\hline & & $\begin{array}{l}\text { Uberhaupt } \\
\text { nicht }\end{array}$ & Ein wenig & $\begin{array}{l}\text { Muttel- } \\
\text { masig }\end{array}$ & Ziemlich & Außerst \\
\hline 7 & We gut können Sie sich kenzentrieren? & 1 & 2 & 3 & 4 & 5 \\
\hline 8 & $\begin{array}{l}\text { We sicher fuhlen Sie sich in threm taglichen } \\
\text { Leben? }\end{array}$ & 1 & 2 & 3 & 4 & 5 \\
\hline 9 & $\begin{array}{l}\text { We gesund sind die Umweltbedingungen in threm } \\
\text { Wohngebiet? }\end{array}$ & 1 & 2 & 3 & 4 & 5 \\
\hline
\end{tabular}

In den folgenden Fragen geht es darum, in welchem Umfang Sie während der vergangenen zwei Wochen bestimmte Dinge erlebt haben oder in der Lage waren, bestimmte Dinge zu tun 


\section{UNIVERSITÄTSMEDIZIN $: \mathbf{M M G}$
GOTTIINGEN $: \mathbf{M G}$}

\begin{tabular}{|c|c|c|c|c|c|c|}
\hline & & $\begin{array}{l}\text { Uber- } \\
\text { haupt } \\
\text { nicht }\end{array}$ & Eher nicht & Halbwegs & $\begin{array}{l}\text { Uber- } \\
\text { wiegend }\end{array}$ & Voling \\
\hline 10 & $\begin{array}{l}\text { Haben Sie genug Energie fur das tăgliche } \\
\text { Leben? }\end{array}$ & 1 & 2 & 3 & 4 & 5 \\
\hline 11 & Konnen Sie thr Aussehen akzeptieren? & 1 & 2 & 3 & 4 & 5 \\
\hline 12 & $\begin{array}{l}\text { Haben Sie genug Geld, um thre Bedirfnisse } \\
\text { erfulion zu können? }\end{array}$ & 1 & 2 & 3 & 4 & 5 \\
\hline 13 & $\begin{array}{l}\text { Haben Sie Zugang zu den Informationen, die Sie } \\
\text { fur das tăgliche Leben brauchen? }\end{array}$ & 1 & 2 & 3 & 4 & 5 \\
\hline 14 & $\begin{array}{l}\text { Haben Sie ausreichend Molichkeiten zu } \\
\text { Freizeitaktivitaten? }\end{array}$ & 1 & 2 & 3 & 4 & 5 \\
\hline & & $\begin{array}{l}\text { Sehr } \\
\text { schlecht }\end{array}$ & schlechit & $\begin{array}{l}\text { Mittel- } \\
\text { maBig }\end{array}$ & Qut & Sehr gut \\
\hline 15 & Wie gut können Sie sich fortbewegen? & 1 & 2 & 3 & 4 & 5 \\
\hline
\end{tabular}

In den folgenden Fragen geht es darum, wie zufrieden, glücklich oder gut Sie sich während der vergangenen zwei Wochen hinsichtlich verschiedener Aspekte thres Lebens gefuhit haben

\begin{tabular}{|c|c|c|c|c|c|c|}
\hline- & & $\begin{array}{l}\text { Sehr un- } \\
\text { zufrieden }\end{array}$ & $\begin{array}{c}\text { Un- } \\
\text { zufrieden }\end{array}$ & $\begin{array}{l}\text { Weder } \\
\text { zufrieden } \\
\text { noch un- } \\
\text { zufrieden }\end{array}$ & Zutrieden & $\begin{array}{l}\text { Sehr } \\
\text { zufrieden }\end{array}$ \\
\hline 16 & We zulfieden sind Sie mit threm Schlan? & 1 & 2 & 3 & 4 & 5 \\
\hline 17 & $\begin{array}{l}\text { Wie zufrieden sind Sie mit threr Fahigkeit, } \\
\text { alltăgliche Dinge erledigen zu konnen? }\end{array}$ & 1 & 2 & 3 & 4 & 5 \\
\hline 18 & Wie zufrieden sind Sie mit threr Arbeits fahigkeit? & 1 & 2 & 3 & 4 & 5 \\
\hline 19 & Wie zufrieden sind Sie mitsich selbst? & 1 & 2 & 3 & 4 & 5 \\
\hline 20 & $\begin{array}{l}\text { We zutrieden sind Sie mit linren pers dnlichen } \\
\text { Beziehungen? }\end{array}$ & 1 & 2 & 3 & 4 & 5 \\
\hline 21 & Whe zufrieden sind Sie mit hrem Sexualleben? & 1 & 2 & 3 & 4 & 5 \\
\hline
\end{tabular}




\section{UNIVERSITÄTSMEDIZIN $=\mathbf{W M G}$
GOOTTINGEN $=\mathbf{M G}$}

\begin{tabular}{|c|c|c|c|c|c|c|}
\hline & & $\begin{array}{l}\text { Sehr un- } \\
\text { zufrieden }\end{array}$ & $\begin{array}{l}\text { Un- } \\
\text { zufriaden }\end{array}$ & $\begin{array}{l}\text { Weder } \\
\text { zufrieden } \\
\text { noch un- } \\
\text { zufrieden }\end{array}$ & Zufrieden & $\begin{array}{l}\text { Sehr } \\
\text { zufrieden }\end{array}$ \\
\hline 22 & $\begin{array}{l}\text { Wie zufrieden sind Sie mit der Unterstitzung } \\
\text { durch thre Freunde? }\end{array}$ & 1 & 2 & 3 & 4 & 5 \\
\hline 23 & $\begin{array}{l}\text { Wie zulrieden shd sie } \mathrm{mil} \text { thren } \\
\text { Wohnbedingungen? }\end{array}$ & 1 & 2 & 3 & 4 & 5 \\
\hline 24 & $\begin{array}{l}\text { Wie zufrieden sind Sie mit Ihren Moglichikeiten, } \\
\text { Gesundheitsdienste in Anspruch nehmen zu } \\
\text { konnen? }\end{array}$ & 1 & 2 & 3 & 4 & 5 \\
\hline 25 & $\begin{array}{l}\text { Wie zutrieden sind Sie mitden } \\
\text { Beforderungs mitteln, die Thnen zur Verfiggung } \\
\text { stehen? }\end{array}$ & 1 & 2 & 3 & 4 & 5 \\
\hline
\end{tabular}

In den folgenden Fragen geht es darum, wie of sich während der vergangenen zwei Wochen bei Ihnen negative Gefuhle eingestellt haben, wie zum Beispiel Angst oder Traurigkeit

\begin{tabular}{|c|l|c|c|c|c|c|}
\hline 26 & $\begin{array}{l}\text { Niemals } \\
\begin{array}{l}\text { Wraurigkig haben Sie negative Gefuhle wie } \\
\text { Depression? }\end{array}\end{array}$ & 1 & 2 & 3 & 4 & Imeiflung, Angst oder \\
\hline
\end{tabular}

Hat Ihnen jemand beim Ausfullen dieses Fragebogens geholfen?

Wie lange hat es gedauert, den Fragebogen auszufullen?

Minuten

\section{Haben Sie irgendweiche Anmerkungen zu diesem Fragebogen?}

\begin{tabular}{|ll|}
\hline Patientennummer: $\ldots \ldots$ & Datum: $\ldots+\cdots$ \\
Geburtsdatum: & Praxiscode: $\ldots \ldots+\ldots$ \\
\hline
\end{tabular}

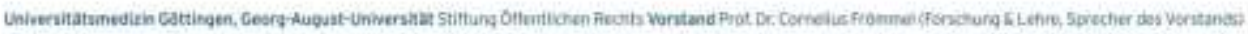

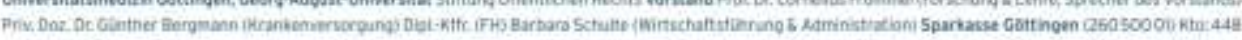




\subsubsection{Hospital Anxiety and Depression Scale - Deutsche Version (HADS-D)}

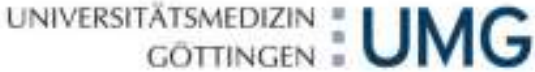

\begin{tabular}{ll}
\hline $\begin{array}{l}\text { Patientennummer: } \\
\text { Geburtsdatum: }\end{array}$ & $\begin{array}{l}\text { Datum: } \\
\text { Praxiscode: } \ldots\end{array}$ \\
\hline & HADS-D \\
(Herman, Buss \& Snaith 1995)
\end{tabular}

Sie werden von uns wegen korperticher Beschwerden untersucht und behandelt. Zur vollstăndigen Beurtelung Ihrer vermuteten oder bereits bekannten Erkrankung bitten wir Sie im vorliegenden Fragebogen um einige persőnliche Angaben. Man weiß heute, dass köpertiche Krankheit und seelisches Befinden oft eng zusammenhangen. Deshalb beziehen sich die Fragen ausdrücklich auf thre allgemeine und seelische Verfassung. Die Beantwortung ist selbstverständlich freiwilig. Wir bitten Sie jedoch, jede Frage zu beantworten, und zwar so, wie es flir Sie personlich in der letzten Woche am ehesten zutrat. Machen Sie bitte nur ein Kreuz pro Frage und lassen Sie bitte keine Frage aus. Oberlegen Sie bitte nicht lange, sondern wahlen Sie die Antwort aus, die thnen auf Anhieb am zutreffendsten erscheint! Alle Ihre Antworten unterliegen der arztlichen Schweigepflicht.

Ich fuhle mich angespannt oder überreizt
a meistens
a of
- won Zeit zu Zeit/gelegentlich
a aberhaupt nicht

Ich kann mich heute noch so freuen wie fruher
- ganz genau so
(] nicht ganz so sehr
a nur noch ein wenig
a kaum oder gar nicht

Mich überkommt eine àngstliche Vorahnung, dass etwas Schreckliches passieren könnte
- ja, sehr stark
a ja, aber nicht allzu stark
- etwas, aber es macht mir keine Somen
a überhaupt nicht

Ich kann lachen und die lustige Seite der Dinge sehen
- ja, so viel wie immer
- nicht mehr ganz so viel
a inzwischen viel weniger
口 überhaupt nicht

Mir gehen beunruhigende Gedanken durch den Kopf
a einen Großteil der Zei
a verhaltrnismäßig of
a von Zeit zu Zeit, aber nicht allzu oft
a nur gelegentlich/nie

Ich fuhle mich glucklich
a überhaupt nicht
a selten
a manchmal
a meistens 


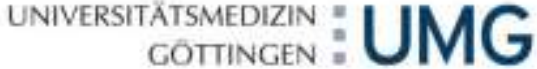

Ich kann behaglich dasitzen und mich entspannen

a ja, naturlich

a gewöhnlich schon

a nicht oft

a überhaupt nicht

Ich fuhle mich in meinen Aktivitàten gebremst
a fast immer
a sehr of
a manchmal
- uberhaupt nicht

Ich habe manchmal ein ängstliches Gefühl in der Magengegend
a überthaupt nicht
a gelegentlich
a ziemlich of
$\square$ sehr of

Ich habe das Interesse an meiner äußeren Erscheinung verloren
a ja, stimmt genau
a ich kümmere mich nicht so sehr darum, wie ich sollte
[ mŏglicherweise kümmere ich mich zu wenig darum
- ich kümmere mich so viel darum we immer

Ich fühle mich rastios, muss immer in Bewegung sein
a ja, tatsachlich sehr
¿ ziemlich
a nicht sehr
aberhaupt nicht

Ich blicke mit Freude in die Zukunft
a ja, sehr
a eher weniger als truher
- viel weniger als fruher
a kaum bis gar nicht

Mich uberkommt plotzlich ein panikartiger Zustand
- ja, tatsachlich sehr of
a ziemlich of
a nicht sehr of
aberhaupt nicht

Ich kann mich an einem guten Buch, einer Radio- oder Fernsehsendung freuen
口 ot
a manchmal
a eher selten
a sehr seiten 


\subsubsection{Soziodemografischer Fragebogen}

\begin{tabular}{|ll|}
$\begin{array}{l}\text { Patientennummer: } \\
\text { Geburtsdatum: }\end{array}$ & Datum: \\
\hline SOZODEMOGRode: \\
(Steinbüchel et al, 2010)
\end{tabular}

1 Geschlecht:

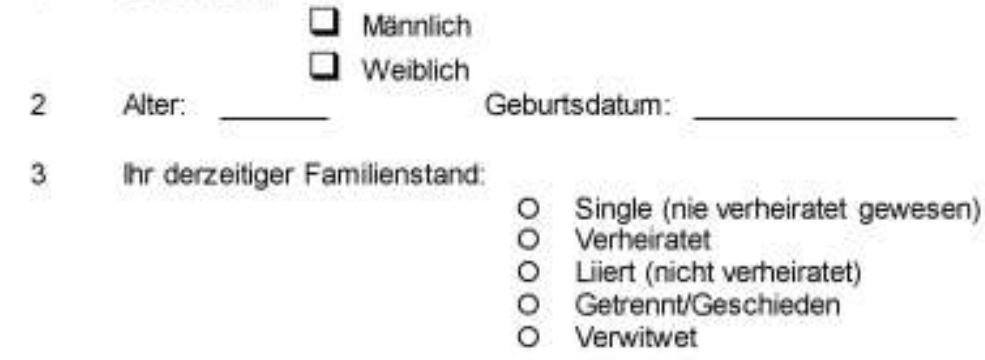

4a Ihr hochster Ausbildungsgrad:
- Grundschule
- Sekundarschule (Haupt-, Realschule, Gymnasium)
- Lehre
- Fachschulabschluss
- Fachhochschul-/Universitätsabschluss
- Sonstiges: Bitte kurz beschreiben

Wie alt waren Sie zum Zeitpunkt Ihres Schulabschlusses?

4b Was ist lihr derzeitiger Beruf?

4c. Bitte kreuzen Sie alle der folgenden Aussagen an, die auf Sie zutreffen.
- Volizeit beschäftigt
- Teilzeit beschaftigt
- Selbstăndig tătig
- Ehrenamtlich tatig
- Arbeitslos
- Pensioniert/berentet
- Dauerhaft arbeitsunfahig
- Student
- Hausfrau
- Kinder betrevend
- Sonstiges: Bitte kurz beschreiben 


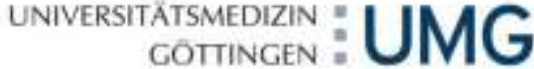

- Zuhause (unabhangig)

- Zuhause (nicht unterstutzt von Familie, Pflegepersonal oder Partner)

- Zuhause (unterstutzt von Familie, Pflegepersonal oder Partner)

- In einer Wohnung mit Betreuung/hauslicher Pflege

- In einem Pflegeheim

- In einem Krankenhaus

- Sonstiges:

Bitte kurz beschreiben

Sind Sie bei den folgenden Aktivitäten auf die Hilfe von anderen angewiesen? Bitte kreuzen Sie die Nummer an, die am besten auf Sie zutrifft.

$\begin{array}{ll}\text { Keine Hilfe } & \text { Ständig } \\ \text { notwendig } & \text { Hilfe } \\ \text { notwendig }\end{array}$

\begin{tabular}{|l|l|l|l|l|l|}
\hline $\begin{array}{l}\text { Grundlegende personliche Bedurfnisse (z. B. auf die } \\
\text { Toilette gehen, sich waschen, sich anziehen, essen, ...) }\end{array}$ & 1 & 2 & 3 & 4 & 5 \\
\hline $\begin{array}{l}\text { Grundlegende Mobilität, Beweglichkeit } \\
\text { (z. B. aufstehen, gehen, Treppen steigen, ...) }\end{array}$ & 1 & 2 & 3 & 4 & 5 \\
\hline $\begin{array}{l}\text { Altagliche Aktivitaten } \\
\text { (z. B. telefonieren, kochen, Wasche waschen, putzen, }\end{array}$ & 1 & 2 & 3 & 4 & 5 \\
\hline $\begin{array}{l}\text { Transport } \\
\text { (z. B. offentliche Verkehrsmittel, Ausfluge,...) }\end{array}$ & 1 & 2 & 3 & 4 & 5 \\
\hline $\begin{array}{l}\text { Organisation und Verwaltung } \\
\text { (z. B. Zahlungen, offizielle Briefe, Termine, ...) }\end{array}$ & 1 & 2 & 3 & 4 & 5 \\
\hline
\end{tabular}

7a Gehen Sie regelmaßig Freizeitaktivitaten nach?

$7 \mathrm{~b} \quad$ Falls ja, weichen?

- Internet, Email

- Kulturelle Aktivitäten ( $z$. B. Ausstellungen, Konzerte, Lesungen, ...)

- Physische Aktivitaten (z. B. Schwimmen, Spazieren gehen, im Sportverein trainieren, ...)

- Hobbys (z. B. Stricken, im Garten arbeiten, ...)

- Musik (z. B, ein Instrument spielen, Singen, Musik hören, ....)

- Unter Leute gehen (z. B. Party, Club, Kneipe, ....)

- Sonstiges: Bitte kurz beschreiben 


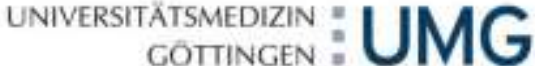

8 Wie oft haben Sie mit den folgenden Personen Kontakt?

Bitte kreuzen Sie die Nummer an, die am besten auf Sie zutrifft.

\begin{tabular}{|l|l|l|l|l|l|}
\hline & \multicolumn{3}{c}{ nie } & \multicolumn{3}{c|}{ täglich } \\
\hline Eltern & 1 & 2 & 3 & 4 & 5 \\
\hline Ehe-/Partner/-in & 1 & 2 & 3 & 4 & 5 \\
\hline Bruder oder Schwester & 1 & 2 & 3 & 4 & 5 \\
\hline Kinder, Enkel & 1 & 2 & 3 & 4 & 5 \\
\hline Anderen engen Familienmitglieder & 1 & 2 & 3 & 4 & 5 \\
\hline Engen Freunde/-innen & 1 & 2 & 3 & 4 & 5 \\
\hline $\begin{array}{l}\text { Bekannte/r (z. B. Nachbar, } \\
\text { Ladenbesitzer) }\end{array}$ & 1 & 2 & 3 & 4 & 5 \\
\hline
\end{tabular}

9 Sind Sie generell der Meinung. dass Sie derzeitig gesund sind, oder nicht?

$$
\begin{aligned}
& \text { Gesund } \\
& \text { Dicht gesund }
\end{aligned}
$$

Rauchen Sie Zgaretten?

O Derzeitige/r Raucher/-in $\rightarrow$ Wie viele Zgaretten pro Woche?

O Frühere/r Raucher/-in $\rightarrow$ Wie lange schon haben Sie aufgehört?

O Ne geraucht

11a Sind in den letzten 6 Monaten für Sie
einschneidende Lebensereignisse vorgefallen?
$\square$ ja
$\square$ nein

11b Wennja, welche einschneidenden Lebensereignisse sind vorgefallen:

- Tod einer nahe stehenden Person (Familienmitglied oder Freund)

- Emste Krankheit einer nahe stehenden Person (Familienmitglied oder Freund)

- Diagnose einer ernsten Krankheit außer Ihrer Hirnverletzung

- Wichtiger Geburtstag

- Geburt eines Kindes

- Hochzeit

- Pensionierung/Arbeitslosigkeit

- Scheidung/Trennung

- Veränderungen in threm Beruf

- Umzug

- Verlust von Geld/wichtigen Gütern, Bankrott

- Stressige oder wichtige Veranderung der Freizeitaktivitaten

- Sonstiges: Bitte kurz beschreiben 


\subsection{Abkürzungsverzeichnis}

$\begin{array}{lll}\text { DMF-T } & = & \text { Decayed-Missing-Filled Teeth Index } \\ \text { HADS-D } & = & \text { Hospital Anxiety and Depression Scale- Deutsche Version } \\ \text { LQ } & = & \text { Lebensqualität } \\ \text { MLQ } & = & \text { mundgesundheitsbezogene Lebensqualität } \\ \text { MMP } & = & \text { Matrix-Metalloproteasen } \\ \text { MW } & = & \text { Mittelwert } \\ \text { N } & = & \text { Anzahl } \\ \text { OHIP } & =\quad \text { Oral Health Impact Profile } \\ \text { Pcheck-12 } & = & \text { Parodontitis-Checkliste } \\ \text { PSI } & = & \text { Parodontaler Screening-Index } \\ \text { PMN } & = & \text { polymorphkernige neutrophile Granulozyten } \\ \text { SD } & = & \text { Standardabweichung } \\ \text { SF-36 } & = & \text { Whort-Form Health Survey } \\ \text { WHO } & & \text { World Health Organization Quality of Life Scale } \\ \text { WHOQOL-BREF } & \end{array}$




\subsection{Abbildungsverzeichnis}

Abbildung 1: Ätiologie und Pathogenese der Parodontitis nach Page und Kornman......... 7

Abbildung 2: Modell zur mundgesundheitsbezogenen Lebensqualität (MLQ),

gesundheitsbezogenen Lebensqualität und allgemeinen Lebensqualität nach

John und Micheelis (2003).

Abbildung 3: Die Hauptkomponenten der mundgesundheitsbezogenen Lebensqualität nach Inglehart und Bagramian (frei übersetzt) (Inglehart \& Bagramian, 2002)

Abbildung 4: WHO-Sonde mit Skalierung (eigenes Bild)

Abbildung 5: Zuordnung in die zwei Grunddimensionen: körperliche und psychische

Gesundheit

Abbildung 6: Verteilung der PSI-Werte der einzelnen Sextanten in Gruppe A (PSI 1-2)...32

Abbildung 7: Verteilung der PSI-Werte der einzelnen Sextanten in Gruppe B (PSI 3-4)...32

Abbildung 8: Graphische Darstellung zur Einschätzung der Patienten ihres allgemeinen und Mundgesundheitszustands in beiden Gruppen 35

Abbildung 9: Prävalenz von generell vorhandenen Mundgesundheitsproblemen (d. h.

Minimalwert von 1 „kaum“) aus dem OHIP-G14 in beiden Gruppen. 


\subsection{Tabellenverzeichnis}

Tabelle 1: Erhebungszweck und Durchführungsmodalität der sieben Fragebögen der eingesetzten Fragebogenbatterie

Tabelle 2: Bewertungsgrade des PSI .23

Tabelle 3: Patientencharakteristika in beiden Vergleichsgruppen 29

Tabelle 4: Mittelwerte, Standardabweichung, minimale und maximale Werte der Studiengruppen in Bezug auf den dentalen Befund 30

Tabelle 5: Absolute Häufigkeit der maximalen PSI-Werte in den beiden Studiengruppen 31 Tabelle 6: Verteilung der einzelnen Parodontitis-spezifischen Symptome in beiden Gruppen ..... 33

Tabelle 7: Verteilung der zahnärztlichen Vorsorge in beiden Gruppen 34

Tabelle 8: Profil der Antworten des OHIP-G14 absolut und in Prozent in Gruppe A (PSI 1-

2) und Gruppe B (PSI 3-4) 36

Tabelle 9: Prävalenzwerte der berichteten Beeinträchtigungen bei Berücksichtigung jeder positiven Antwort im OHIP-G14 getrennt nach den Studiengruppen 38

Tabelle 10: Am häufigsten genannte Probleme (ab 6 Nennungen) aus dem Fragebogen „Allgemeiner Gesundheitszustand“ absolut und in Prozent für beide Studiengruppen

Tabelle 11: Mittelwerte, Standardabweichung, minimale und maximale Werte der KSK bzw. PSK in beiden Studiengruppen und der deutschen Normstichprobe......39

Tabelle 12: Mittelwerte, Standardabweichung, minimale und maximale Werte in beiden Studiengruppen und in der Normbevölkerung in den vier Domänen des WHOQOL-BREF

Tabelle 13: Mittelwerte, Standardabweichung, minimale und maximale Werte in beiden Studien und in der Normbevölkerung im HADS-D

Tabelle 14: Spearmansche Korrelation und Signifikanzwerte (einseitig) von Mundgesundheit / Allgemeiner Gesundheit mit gesundheitsbezogener LQ / mundgesundheitsbezogener LQ.

Tabelle 15: Spearmansche Korrelation und Signifikanzwerte (zweiseitig) von Angst /

Depression / Alter mit gesundheitsbezogener LQ / mundgesundheitsbezogener LQ

Tabelle 16: Spearmansche Korrelation und Signifikanzwerte (einseitig) von Mundgesundheit / Allgemeiner Gesundheit mit gesundheitsbezogener LQ / mundgesundheitsbezogener LQ (getrennt nach Geschlecht)

Tabelle 17: Spearmansche Korrelation und Signifikanzwerte (zweiseitig) von Angst /

Depression / Alter mit gesundheitsbezogener LQ /

mundgesundheitsbezogener LQ (getrennt nach Geschlecht) 


\section{$8 \quad$ Literaturverzeichnis}

Ainamo J, Barmes D, Beagrie G, Cutress T, Martin J, Sardo-Infirri J (1982): Development of the World Health Organization (WHO) community periodontal index of treatment needs (CPITN). Int Dent J $\underline{32}(3), 281-291$

Al Habashneh R, Khader YS, Salameh S (2012): Use of the Arabic version of Oral Health Impact Profile-14 to evaluate the impact of periodontal disease on oral healthrelated quality of life among Jordanian adults. J Oral Sci $\underline{54}(1), 113-120$

Allen PF (2003): Assessment of oral health related quality of life. Health Qual Life Outcomes $\underline{1}, 40$

Angermeyer MC, Kilian R, Matschinger H: WHOQOL-100 und WHOQOL-BREF Handbuch für die deutschsprachige Version der WHO Instrumente zur Erfassung von Lebensqualität. Hogrefe Verlag, Göttingen 2000

Apolone G, Mosconi P (1998): The Italian SF-36 Health Survey: translation, validation and norming. J Clin Epidemiol 51(11), 1025-1036

Armitage GC (1999): Development of a classification system for periodontal diseases and conditions. Ann Periodontol $\underline{4}(1), 1-6$

Atchison KA, Dolan TA (1990): Development of the Geriatric Oral Health Assessment Index. J Dent Educ $\underline{54}(11), 680-687$

Baelum V, Fejerskov O, Karring T (1986): Oral hygiene, gingivitis and periodontal breakdown in adult Tanzanians. J Periodontal Res 21 (3), 221-23

Bernabé E, Marcenes W (2010): Periodontal disease and quality of life in British adults. J Clin Periodontol 37(11), 968-972

Bjelland I, Dahl AA, Haug TT, Neckelmann D (2002): The validity of the Hospital Anxiety and Depression Scale. An updated literature review. J Psychosom Res $\underline{52(2), 69-77}$

Bjorner JB, Thunedborg K, Kristensen TS, Modvig J, Bech P (1998): The Danish SF-36 Health Survey: translation and preliminary validity studies. J Clin Epidemiol 51(11), 991-999

Bullinger M (1995): German translation and psychometric testing of the SF-36 Health Survey: preliminary results from the IQOLA Project. International Quality of Life Assessment. Soc Sci Med 41(10), 1359-1366

Bullinger M, Kirchberger I: SF-36 Fragebogen zum Gesundheitszustand Handanweisung. Hogrefe Verlag für Psychologie, Göttingen 1998 
Bullinger M, Ravens-Sieberer U, Siegrist J: Gesundheitsbezogene Lebensqualität in der Medizin - eine Einführung. In: Bullinger M, Siegrist J, Rabens-Sieberer U (Hrsg.): Lebensqualitätsforschung aus medizinpsychologischer und -soziologischer Perspektive. Jahrbuch der Medizinschen Psychologie, Band 18. Hogrefe Verlag, Göttingen 2000, 11-24

Casanova L, Hughes FJ, Preshaw PM (2014): Diabetes and periodontal disease: a twoway relationship. Br Dent J 217(8), 433-437

Costanza R, Fisher B, Ali S, Beer C, Bond L, Boumans R, Danigelis NL, Dickinson J, Elliott C, Farley J (2008): An Integrative Approach to Quality of Life Measurement, Research, and Policy. SAPIENS 1(1), 17-21

Cunha-Cruz J, Hujoel PP, Kressin NR (2007): Oral health-related quality of life of periodontal patients. J Periodontal Res $\underline{42}(2), 169-176$

Darveau RP, Tanner A, Page R (1997): The microbial challenge in periodontitis.

Periodontol $2000 \underline{14}, 12-32$

Davis DM, Fiske J, Scott B, Radford DR (2000): The emotional effects of tooth loss: a preliminary quantitative study. Br Dent J $\underline{188(9), 503-506}$

Deutsche Gesellschaft für Parodontologie: Klassifikation der Parodontalerkrankungen. Quintessenz Verlag, Berlin 2002

Ebbinghaus B, Noll HB, Bahle T, Wendt C, Scheuer A: VFA-Report Lebensqualität 2006. Mannheimer Zentrum für Europäische Sozialforschung, Mannheim 2006

Ellert U, Bellach BM (1999): Der SF-36 im Bundes-Gesundheitssurvey - Beschreibung einer aktuellen Normstichprobe. Gesundheitswesen 61(2), 184-190

Ellert U, Kurth BM (2013): Gesundheitsbezogene Lebensqualität bei Erwachsenen in Deutschland - Ergebnisse der Studie zur Gesundheit Erwachsener in Deutschland (DEGS1). Bundesgesundheitsbl $\underline{56}(5), 643-649$

Flemmig TF (1999): Periodontitis. Ann Periodontol 4(1), 32-37

Fotedar S, Sharma KR, Fotedar V, Bhardwaj V, Chauhan A, Manchanda K (2014):

Relationship between oral health status and oral health related quality of life in adults attending H.P. Government Dental College, Shimla, Himachal PradeshIndia. Oral Health Dent Manag 13(3), 661-665

Gerritsen AE, Allen PF, Witter DJ, Bronkhorst EM, Creugers NH (2010): Tooth loss and oral health-related quality of life: a systematic review and meta-analysis. Health Qual Life Outcomes $\underline{8}, 126$

Glavind L, Löe H (1967): Errors in the clinical assessment of periodontal destruction. J Periodontal Res $\underline{2}(3), 180-184$

Haffajee AD, Socranksky SS (1994): Microbial etiological agents of destructive periodontal diseases. Periodontol $2000 \underline{5}, 78-111$ 
Haffajee AD, Socranksky SS (2005): Microbiology of periodontal diseases: introduction. Periodontol $2000 \underline{38}, 9-12$

Haffajee AD, Socranksky SS (2006): Introduction to microbial aspects of periodontal biofilm communities, development and treatment. Periodontol $2000 \underline{42}$, 7-12

Hellwig E, Klimek J, Attin T: Einführung in die Zahnerhaltung. 3. Auflage; Urban \& Fischer Verlag, München 2003

Herrmann-Lingen C, Buss U, Snaith RP: Hospital Anxiety and Depression Scale Deutsche Version (HADS-D). 3. aktualisierte und neu normierte Auflage; Verlag Hans Huber, Bern 2011

Holtfreter B, Kocher T, Hoffmann T, Desvarieux M, Micheelis W (2010): Prevalence of periodontal disease and treatment demands based on a German dental survey (DMX IV). J Clin Periodontol 37(3), 211-219

Holtfreter B, Demmer RT, Bernhardt O, Papapanou PN, Schwahn C, Kocher T, Desvarieux M (2012): A comparison of periodontal status in the two regional, population-based studies of SHIP and INVEST. J Clin Periodontol 39(12), 11151124

Hsiung PC, Fang CT, Chang YY, Chen MY, Wang JD (2005): Comparison of WHOQOLBREF and SF-36 in patients with HIV infection. Qual Life Res 14(1), 141-150 Inglehart MR, Bagramian RA: Oral Health-Related Quality of Life: An Introduction. In: Inglehart MR, Bagramian RA (Hrsg.): Oral Health-Related Quality of Life. Quintessence Pub, Carol Stream IL-USA 2002, 1-6

John MT (2004): Demographic factors, denture status and oral health-related quality of life. Community Dent Oral Epidemiol $\underline{32}(2), 125-132$

John MT (2005): Mundgesundheitsbezogene Lebensqualität (MLQ). Zahnärztliche Mitteilungen $\underline{21}, 68-72$

John MT, Micheelis W (2003): Mundgesundheitsbezogene Lebensqualität in der Bevölkerung: Grundlagen und Ergebnisse des Oral Health Impact Profile (OHIP) aus einer repräsentativen Stichprobe in Deutschland. IDZ-Informationen 1(3), 1-28 John MT, Micheelis W (2004): Normwerte mundgesundheitsbezogener Lebensqualtiät für Kurzversionen des Oral Health Impact Profile. Schweiz Monatsschr Zahnmed 114(8), 784-791

John MT, Patrick DL, Slade DG (2002): The German version of the Oral Health Impact Profile - translation and psychometric properties. Eur J Oral Sci 110(6), 425-433 John MT, LeResche L, Koepsell TD, Hujoel P, Miglioretti DL, Micheelis W (2003): Oral health-related quality of life in Germany. Eur J Oral Sci, 111(6), 483-491 
John MT, Micheelis W, Biffar R (2004): Einflussfaktoren mundgesundheitsbezogener Lebensqualität - Validierung einer deutschen Kurzversion des Oral Health Impact Profile (OHIP-G14). Deut Zahnärztl Z $\underline{59}(6)$, 328-333

John MT, Miglioretti DL, LeResche L, Koepsell TD, Hujoel P, Micheelis W (2006): German short forms of the Oral Health Impact Profile. Community Dent Oral Empidemiol 34(4), 277-288

Jordan R: Fünfte Deutsche Mundgesundheitsstudie (DMS V) - Kurzfassung. BZÄK/KZBV, Berlin/Köln 2016

Kachkachishvili ID (2013): Georgian version of the "Oral Health Impact Profile". Georgien Med News $\underline{218}$, 23-28

Konerding U: Gesundheitsbezogene Lebensqualität. In: Lauterbach KW, Schrappe M (Hrsg.): Gesundheitsökonomie, Qualitätsmanagment und Evidence-based Medicine.

2. Auflage; Schattauer, Stuttgart 2004, 160-182

Lindhe J, Hamp SE, Löe H (1973): Experimental periodontitis in the Beagle dog. J Periodontal Res $\underline{8}(1), 1-10$

Listgarten MA (1986): Pathogenesis of periodontitis. J Clin Periodontol 13(5), 418-425 Listgarten MA (1987): Nature of periodontal diseases: Pathogenic mechanisms. J Periodontal Res 22(3), 172-178

Listgarten MA, Schifter CC, Laster L (1985): 3-year longitudinal study of the periodontal status of an adult population with gingivitis. J Clin Periodontol 12(3), 225-238

Liu JY, Pow EH, Chen ZF, Zheng J, Zhang XC, Chen J (2012): The Mandarin Chinese shortened version of Oral Health Impact Profile for partially edentate patients with implant-supported prostheses. J Oral Rehabil 39(8), 591-599

Locker D (1988): Measuring oral health: a conceptual framework. Community Dent Health $\underline{5}(1), 3-18$

Locker D (2004): Oral health and quality of life. Oral Health Prev Dent 2(1), 247-253 Löe H, Theilade E (1965): Experimental Gingivitis in Man. J Periodontol 36, 177-187 Löe H, Anerud A, Boysen H, Morrison E (1986): Natural history of periodontal disease in man. Rapid, moderate and no loss of attachment in Sri Lankan laborers 14 to 46 years of age. J Clin Periodontol 13(5), 431-440

López R, Baelum V (2007): Oral Health Impact of Periodontal Diseases in Adolescents. J Dent Res $\underline{86}(11), 1105-1109$

McGrath C, Bedi R (2001): Can dental attendance improve quality of life?. Br Dent J $\underline{190}(5), 262-265$

McGrath C, Bedi R (2004): A national study of the importance of oral health to life quality to inform scales of oral health related quality of life. Qual Life Res $\underline{13}(4), 813-818$ Micheel HG: Quantitative empirische Sozialforschung. Reinhardt UTB, München 2010 
Micheelis W: Dritte Deutsche Mundgesundheitsstudie (DMS III). Ergebnisse, Trends und Problemanalysen auf der Grundlage bevölkerungsrepräsentativer Stichproben in Deutschland 1997. Deutscher Ärzte-Verlag, Köln 1997

Micheelis W, Schiffner U: Vierte Deutsche Mundgesundheitsstudie (DMS IV). Deutscher Zahnärzte Verlag DÄV, Köln 2006

Min SK, Kim KI, Lee CI, Jung YC, Suh SY, Kim DK (2002): Development of the Korean versions of WHO Quality of Life scale and WHOQOL-BREF. Qual Life Res 11(6), $593-600$

Müller F, von Steinbüchel N (2005): Lebensqualität im Alter und subjektive Mundgesundheit. Quintessenz $\underline{56}(12), 1291-1300$

Needleman I, McGrath C, Floyd P, Biddle A (2004): Impact of oral health on the life quality of periodontal patients. J Clin Periodontol $\underline{31}$ (6), 454-457

$\mathrm{Ng}$ SK, Leung WK (2006): Oral health-related quality of life and periodontal status. Community Dent Oral Epidemiol 34(2), 114-122

Noerholm V, Groenvold M, Watt T, Bjorner JB (2004): Quality of life in the Danish general population-normative data and validity of WHOQOL-BREF using Rasch and item response theory models. Qual Life Res $\underline{13}(2), 531-540$

Noll HH: Konzepte der Wohlfahrtsentwicklung: Lebensqualität und "neue" Wohlfahrtskonzepte. EuReporting Paper Nr. 3; ZUMA, Mannheim 1999

Noll HH: Social Indicators and Quality of Life Research: Background, Achievements and Current Trends. In: Genov N (Hrsg.): Advances in Sociological Knowledge Over Half a Century. ISSC, Paris 2002, 168-206

Nussbaum M, Sen A: The Quality of Life. Clarendon Press, Oxford 1993

OECD: How's Life? 2015: Measuring Well-being. OECD Publishing, Paris 2015

Ohaeri JU, Olusina AK, Al-Abassi AH (2004): Factor analytical study of the short version of the World Health Organization Quality of Life Instrument. Psychopathology $\underline{37(5),}$ 242-248

Ohrn K, Jönsson B (2012): A comparison of two questionnaires measuring oral healthrelated quality of life before and after dental hygiene treatment in patients with periodontal disease. Int J Dent Hyg 10 (1), 9-14

Page RC (1986): Gingivitis. J Clin Periodontol 13(5), 345-355

Page RC (1999): Milestones in periodontal research and the remaining critical issues. $J$ Periodontal Res 34(7), 331-339

Page RC, Kornman KS (1997): The pathogenesis of human periodontitis: an introduction. Periodontol $2000 \underline{14}, 9-11$

Prchala G (2004): Ein Leben voll Qualität. ZM 94(15), 26-31 
Ravens-Sieberer U, Cieza A: Lebensqualitätsforschung in Deutschland: Forschungsstand, Methoden, Anwendungsbeispiele und Implikationen. In: Ravens-Sieberer U, Cieza A (Hrsg.): Lebensqualität und Gesundheitsökonomie in der Medizin: Konzepte Methoden - Anwendung. ecomed, Landsberg 2000, 25-50

Renneberg B, Lippke S: Lebensqualität. In: Renneberg B, Hammelstein P (Hrsg.): Gesundheitspsychologie. Springer, Heidelberg 2006, 29-33

Saito A, Hosaka Y, Kikuchi M, Akamatsu M, Fukaya C, Matsumoto S, Ueshima F, Hayakawa H, Fujinami K, Nakagawa T (2010): Effect of Initial Periodontal Therapy on Oral Health-Related Quality of Life in Patients With Periodontitis in Japan. J Periodontol $\underline{81}(7), 1001-1009$

Shah M, Kumar S (2011): Improvement of Oral Health Related Quality of Life in Periodontitis Patients after Non-Surgical Periodontal Therapy. J Int Oral Health $\underline{3}$, $15-21$

Shanbhag S, Dahiya M, Croucher R (2012): The impact of periodontal therapy on oral health-related quality of life in adults: a systematic review. J Clin Periodontol 39(8), 725-735

Sischo L, Broder HL (2011): Oral Health-related Quality of Life: What, Why, How, and Future Implications. J Dent Res $\underline{90}(11), 1264-1270$

Skevington SM, Lotfy M, O'Connell KA (2004): The World Health Organization's WHOQOL-BREF quality of life assessment: Psychometric properties and results of the international field trial. A Report from the WHOQOL Group. Qual Life Res $\underline{13}(2)$, 299-310

Slade G (1997): Derivation and validation of a short-form oral health impact profile. Community Dent Oral Epidemiol 25(4), 284-290

Slade GD, Spencer AJ (1994): Development and evaluation of the Oral Health Impact Profile. Community Dent Health 11(1), 3-11

Steele JG, Sanders AE, Slade GD, Allen PF, Lahti S, Nuttall N, Spencer AJ (2004): How do age and tooth loss affect oral health impacts and quality of life? A study comparing two national samples. Community Dent Oral Epidemiol 32(2), 107-114

Teles R, Teles F, Frias-Lopez J, Paster B, Haffajee A (2013): Lessons learned and unlearned in periodontal microbiology. Periodontol $2000 \underline{62}(1), 95-162$

Trompenaars FJ, Masthoff ED, Van Heck GL, Hodiamont PP, De Vries J (2005): Content validity, construct validity, and reliability of the WHOQOL-Bref in a population of Dutch adult psychiatric outpatients. Qual Life Res 14(1), 151-160

Von Steinbüchel N, Lischetzke T, Gurny M, Eid M (2006): Assessing quality of life in older people: psychometric properties of the WHOQOL-BREF. Eur J Ageing $\underline{3}, 116-122$. 
Ware JE, Sherbourne CD (1992): The MOS 36-item short-form health survey (SF-36). I. Conceptual framework and item selection. Med Care 30(6), 473-483

WHOQOL Group (1995): The World Health Organization quality of life assessment (WHOQOL): position paper from the World Health Organization. Soc Sci Med $\underline{41}(10), 1403-1409$

WHOQOL Group (1998): Development of the World Health Organization WHOQOL-BREF quality of life assessment. The WHOQOL Group. Psychol Med 28(3), 551-558

Wolf HF, Rateitschak EM, Rateitschak KH: Farbatlanten der Zahnmedizin -

Parodontologie. Band 1; kartonierte Sonderausgabe der 3. Auflage; Georg Thieme Verlag, Stuttgart 2012

World Health Organization - WHO. Verfassung der Weltgesundheitsorganisation. World Health Organization, Genf 1948 


\section{Danksagung}

Mein besonderer Dank gilt Prof. Dr. Rainer Mausberg i.R. und PD Dr. Dirk Ziebolz für die freundliche Überlassung und die sehr gute Betreuung dieser Arbeit. Dabei möchte ich mich herzlich für die lange Ausdauer, unermüdliche Beantwortung meiner Fragen und die Durchsicht der Arbeit bei PD. Dr. Dirk Ziebolz bedanken.

Vielen Dank an Frau Prof. Dr. Nicole von Steinbüchel-Rheinwall aus dem Institut für Medizinische Psychologie und Medizinische Soziologie der Universitätsmedizin Göttingen und Dr. Wolfgang Micheelis vom Institut der deutschen Zahnärzte (IDZ) in Köln für die Hilfe bei der Erstellung des Studiendesigns.

Großer Dank geht auch an Mag. Albert Rosenberger aus dem Institut für Genetische Epidemiologie der Universitätsmedizin Göttingen für die Unterstützung bei der statistischen Auswertung der Daten.

Zudem danke ich allen Patienten, die freundlicherweise freiwillig an dieser Untersuchung teilgenommen haben. 